\title{
High-performance model reduction techniques in computational multiscale homogenization
}

\author{
J.A. Hernández ${ }^{\mathrm{a}, \mathrm{c}, *}$, J. Oliver ${ }^{\mathrm{a}, \mathrm{b}}$, A.E. Huespe ${ }^{\mathrm{b}, \mathrm{d}}$, M.A. Caicedo ${ }^{\mathrm{a}}$, J.C. \\ Cante $^{\mathrm{a}, \mathrm{c}}$ \\ ${ }^{a}$ Centre Internacional de Mètodes Numèrics en Enginyeria (CIMNE), Technical \\ University of Catalonia, Edificio C1, Campus Norte, Jordi Girona 1-3, Barcelona 08034, \\ Spain \\ ${ }^{b}$ E.T.S. d'Enginyers de Camins, Canals $i$ Ports, Technical University of Catalonia, \\ Edificio C1, Campus Norte, Jordi Girona 1-3, Barcelona 08034, Spain \\ ${ }^{c}$ E.T.S. d'Enginyeries Industrial i Aeronàutica de Terrassa, Technical University of \\ Catalonia, C/ Colom, 11, Terrassa 08222, Spain \\ ${ }^{d}$ CIMEC, CONICET, Güemes 3450, 3000 Santa Fe, Argentina
}

\begin{abstract}
A novel model-order reduction technique for the solution of the fine-scale equilibrium problem appearing in computational homogenization is presented. The reduced set of empirical shape functions is obtained using a partitioned version - that accounts for the elastic/inelastic character of the solutionof the Proper Orthogonal Decomposition (POD). On the other hand, it is shown that the standard approach of replacing the nonaffine term by an interpolant constructed using only POD modes leads to ill-posed formulations. We demonstrate that this ill-posedness can be avoided by enriching the approximation space with the span of the gradient of the empirical shape functions. Furthermore, interpolation points are chosen guided, not only by accuracy requirements, but also by stability considerations. The approach is assessed in the homogenization of a highly complex porous metal material. Computed results show that computational complexity is independent of the size and geometrical complexity of the representative volume element. The speedup factor is over three orders of magnitude - as compared with finite element analysis - whereas the maximum error in stresses is less than $10 \%$.
\end{abstract}

\footnotetext{
${ }^{*}$ Corresponding author

Email address: jhortega@cimne.upc.edu (J.A. Hernández)
} 
Keywords: Multiscale, homogenization, model reduction, High-Performance reduced-order model, hyperreduction, POD

\section{Introduction}

\subsection{Motivation and goal}

The major challenge in the macro-scale continuum description of hetcrogencous materials such as composites and polycrystalline metals (that exhibit a clear heterogeneous composition at the the micro-, or meso-, scale, but that can be regarded, for practical purposes, as homogeneous at the the macro-scale) lies in the determination of a constitutive connection, between macro-stresses and macro-strains, that accurately reflects the properties and geometrical arrangement of the distinct phases at the finer scale. It is wellknown [34] that, under the hypotheses of either periodicity or statistical ho-
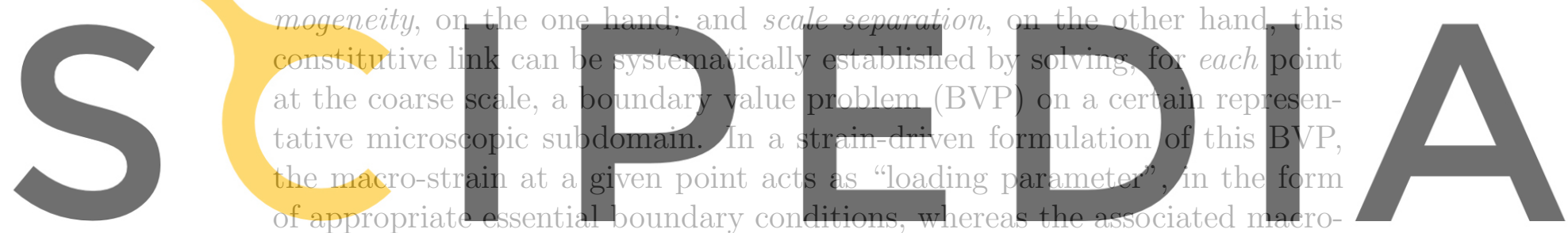

stress is obtained through volume averaging - i.e., homogenization - of the

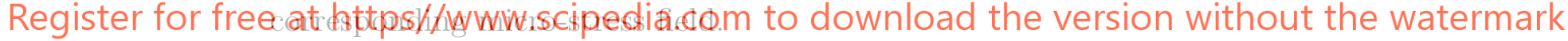

Methods dealing with the solution of this BVP range from purely analytical approaches to direct computational methods, such as the two-level, Finite Element $\left(\mathrm{FE}^{2}\right)$ method [29]. Analytical approaches are computationally inexpensive, but only valid for certain types of geometrically and constitutively simple micro-structures. By contrast, direct computational methods have no other limitation in scope than the imposed by the aforementioned hypotheses of statistical homogeneity and scale separation - in these methods, the microscopic BVP at each coarse-scale point is attacked using no other approximation than the spatial discretization of the pertinent solution strategy, thus, circumventing the need for introducing ad-hoc, simplifying assumptions regarding the topological arrangement of the micro-phases and/or their collective constitutive behavior. Needless to say, the versatility of direct computational homogenization comes at a significant price: its enormous computational cost. 
Between these two extremes (purely analytical and direct computational methods), there are homogenization strategies that can be termed semianalytical, since they combine analytical results with numerical computations. Such is the case of the Transformation Field Analysis (TFA) [25] and variants thereof $[49,50,56,30]$, which are based on the pre-computation of certain characteristic operators (strain localization and influence tensors) using the information obtained from solving a carefully chosen battery of fine-scale BPVs. Although these methods have notably widen the scope of classical analytical approaches - while maintaining their low computational cost-, they are still predicated, to a lesser or greater extent, on ad-hoc assumptions connected with the constitutive description of the involved phases. Consideration of new materials with unstudied compositions using semi-analytical approaches, thus, requires additional research efforts by specialists in the field and eventual modifications of the corresponding mathematical and numerical formulations - in contrast to direct computational homogenzation approaches, such as the $\mathrm{FF}^{2}$ method in which the formulation is "material-independent", and hence more versatile.
The current state of affairs in the field of two-scale homogenization seems
to call, thus, for a unified homogenization approach that combines some-
what the advantages of lirect computational homogenization and analytical

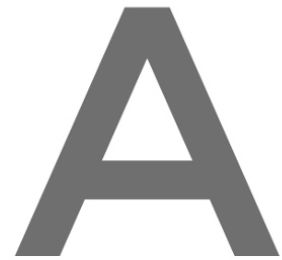

and semi-analytical techniques. It would be desirable to have a homogeniza-

arrive at a method whose mathematical formulation dispenses with ad-hoc, simplifying assumptions related with the composition of the heterogeneous material; i.e, one enjoying the versatility, unrestricted applicability and "userfriendliness" - insofar as it would totally relieve the modeler from the often exceedingly difficult task of visualizing such assumptions — of direct computational homogenization methods. The goal of the present paper is to show that these desirable attributes can be achieved, for arbitrarily complex heterogeneous materials well into the inelastic range, by using the so-called [47] Reduced-Basis (RB) approximation in the solution of the fine-scale BVPs.

\subsection{The reduced-basis method}

Generally speaking, the reduced-basis approximation is a class of Galerkin approximation procedure that employs, as opposed to the FE method, but 
similarly to classical Rayleigh-Ritz solution techniques [19], globally supported basis functions. The main difference with respect to classical RayleighRitz schemes is that these basis functions or modes are not constructed from either polynomials or transcendental functions (sines, cosines ...), but rather are determined from a larger set of previously computed - using the finite element (FE) method or other classical solution techniques - solutions of the BVP at appropriately selected values of the input of interest. These functions are commonly termed empirical basis functions [41], the qualifier empirical meaning "derived from computational experiments".

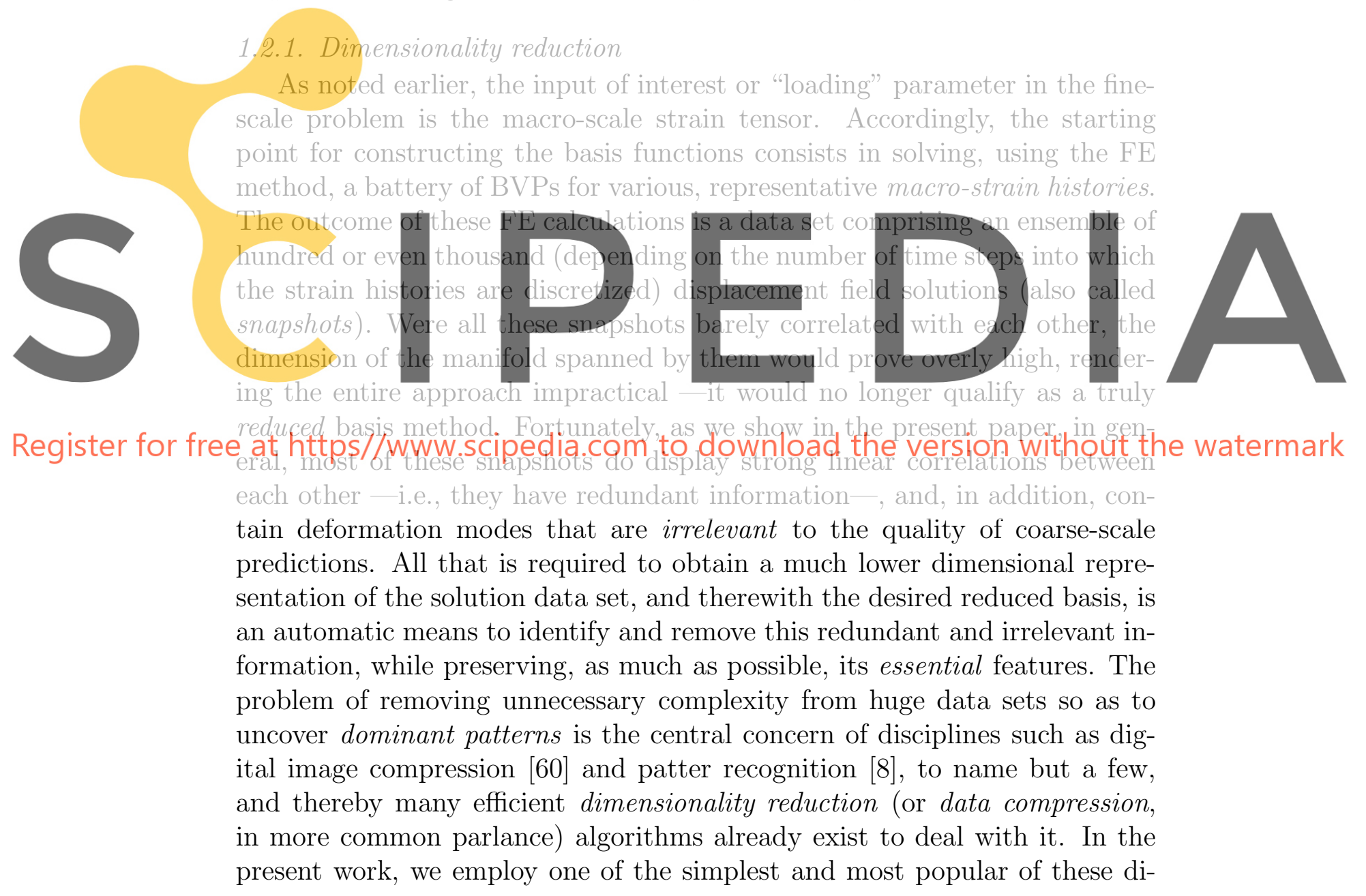


mensionality reduction algorithms: the Proper Orthogonal Decomposition ${ }^{1}$ (POD).

It may be inferred from the above that the proposed homogenization method, like analytical and semi-analytical strategies, does introduce simplifications in solving the fine-scale BVP. However, as opposed to analytical, and to a lesser extent, semi-analytical procedures, these simplifications are not introduced by the modeler, but rather are automatically carried out by the abovementioned dimensionality reduction methods (in an offline stage, prior to the overall multiscale analysis). In other words, in the proposed method, the task of discerning what is essential and what is not ${ }^{2}$ is entirely delegated to the computer itself, and hence, its success does not depend upon the depth of insight, experience, and knowledge base of the modeler - only some discretion is to be exercised in choosing appropriate strain paths for the offline FE analyses. This feature naturally confers the advantages of versatility and "user-friendliness" enjoyed by direct computational methods.
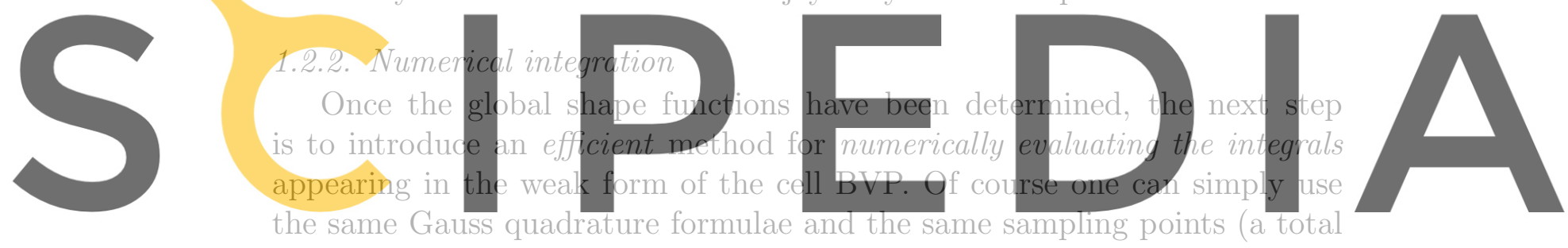

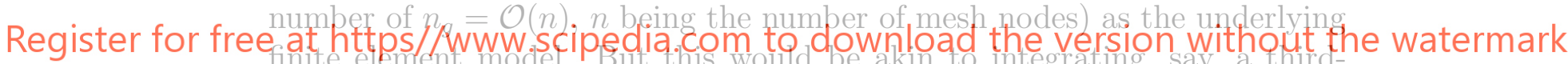
order polynomial function using thousand of sampling points - a profligate waste of computational resources. Since displacement solutions for the cell BVP are constrained to lie in a reduced-order space of dimension $n_{u}<<n$, it is reasonable to expect that the corresponding stresses, internal forces and Jacobians will also reside in reduced-order spaces of dimensions of order $\mathcal{O}\left(n_{u}\right)$, and consequently, only $p=\mathcal{O}\left(n_{u}\right)<<n_{g}$ sampling points would suffice in principle to accurately evaluate the corresponding integrals. The challenging questions that have to be confronted are where to locate these $p$ sampling points and, loosely speaking, how to determine their associated

\footnotetext{
${ }^{1}$ By constraining the cell to deform only into the deformation modes determined by the POD, one automatically obtains a genuine reduced-order model (ROM) of the cell.

${ }^{2}$ Discerning what is essential and what is not is, according to M.Ashby [4], the key to any successful computational model (i.e., one that strikes the right balance between accuracy and simplicity)
} 
weighting functions so that maximum accuracy in the integration is attained.

Approaches found in the model reduction literature that, directly or indirectly, deal with these fundamental questions can be broadly classified either as interpolatory methods [7, 33, 54, 18, 5] or Gauss-type quadrature strategies $[3,39]$. In both types of approaches, the integrand or part of the integrand is approximated by a linear combination of a reduced set of empirical modes. In interpolatory approaches, the coefficients in this approximation are obtained by interpolation at a set of pre-selected sampling points; the criterion for choosing the location of such points is the minimization of the interpolation error over the finite element snapshots. In Gauss-type quadrature procedures, on the other hand, the selection of sampling points and the calculation of the accompanying weighting factors are simultaneously carried out, guided by a criterion of minimum integration error over the snapshots.

In the BVP under consideration, the output of interest is the volume average of the stresses over the cell domain and, therefore, accuracy is required not only in the integration of the equilibrium equation, but also on the approximation of the stresses themselves. This is the reason why attention is
focused here on interpolatory integration strategies, the variable subject to
spatial interpolation being precisely the stresses.
1.3. Originality of this work

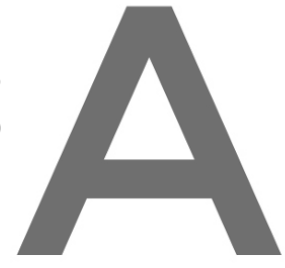

Register for free at hittideg of exploiting the synergistic combination of multiscale modthe the watermark

ing and reduced basis approximation is admittedly not new. In the specific

context of two-scale homogenization, it has been recently explored by Boy-

aval [10], Yvonnet et al. [62], and Monteiro et al. [51]. Traces of this idea can also be found in articles dealing with more general hierarchical multiscale techniques - that do not presuppose either scale separation or periodicity/statistical homogeneity, or both-, namely, in the multiscale finite element method [53, 26, 27], in the heterogeneous multiscale method [2, 1], and in multiscale approaches based on the Proper Generalized Decomposition (PGD)[21]. However, it should be noted that none of the above cited papers confronts the previously described, crucial question of how to efficiently integrate the resulting reduced-order equations, simply because, in most of them $[10,53,26,27,2,1]$, integration is not an issue - the fine-scale BVPs addressed in these works bear an affine relation with the corresponding coarse-scale, input parameter, as in linear elasticity, and, consequently, all integrals can be pre-computed, i.e., evaluated offline, with no impact in the online computational cost. Thus, the development of reduced-order models 
endowed with efficient, mesh-size independent integration schemes - able to handle any material composition - is a research area that, to the best of the authors' knowledge, still remains uncharted.

\subsubsection{Main original contributions}

The theory underlying reduced-order models (ROMs) that incorporate efficient interpolatory integration schemes is still at its embryonic stage of development — the first general proposal for parametrized BVPs dates back to 2004 [7] — and many fundamental issues remain to be addressed. Foremost among these is the crucial question of well-posedness of the resulting system of algebraic equations: does the replacement of the integrand, or nonaffine term in the integrand, by a reduced-order interpolant always lead to a well-posed, discrete problem ? Examination of the reduced basis literature indicates that apparently no researcher has so far been confronted with illposed reduced-order equations, a fact that might certainly promote the view
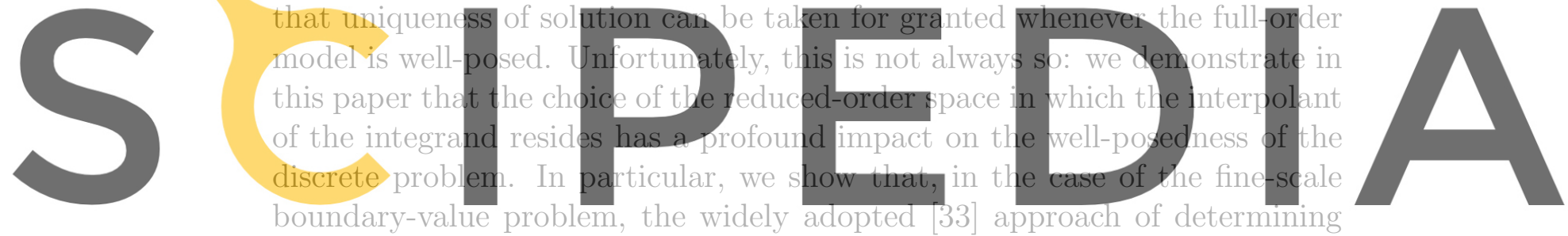

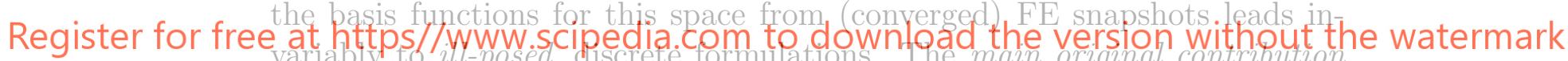

of the present work to the field of reduced-order modeling is the development of an interpolatory integration method that safely overcomes this type of illposedness. The gist of the method is to expand the interpolation space so that it embraces, aside from the span of the POD stress basis functions, the space generated - and herein lies the novelty - by the gradient of the (reduced-order) shape functions. Furthermore, it is shown that, in contrast to the situation encountered when using standard interpolatory schemes in other parametrized BVPs [33], in the BVP under consideration, the number and particular placement of sampling points within the integration domain influence notably the spectral properties (positive definiteness) of the Jacobian matrix of the governing equation, and therefore, the convergence characteristics of the accompanying Newton-Raphson solution algorithm. Another innovative ingredient of the present paper is a points selection algorithm that does acknowledge this peculiarity and chooses the desired sampling points guided, not only by accuracy requirements (minimization of the interpolation 
error over the FE stress snapshot), but also by stability considerations.

\section{RVE equilibrium problem}

In this section, we present the variational statement and finite element discretization of the fine-scale equilibrium problem, which, recall, is the parameterized BVP we wish to efficiently solve using the reduced-basis approximation.

\subsection{Preliminaries}

Let $\Omega \subset \mathbb{R}^{d}(d=2,3)$ be a subvolume of characteristic length $l<<l_{M}$ ( $l_{M}$ is the characteristic length of the macro-continuum $\Omega_{M}$, see Figure 1) that is representative of the heterogeneous material as a whole. In microstructures that exhibit statistical homogeneity, this domain receives the name of Representative Volume Element (RVE), whereas in micro-structures that
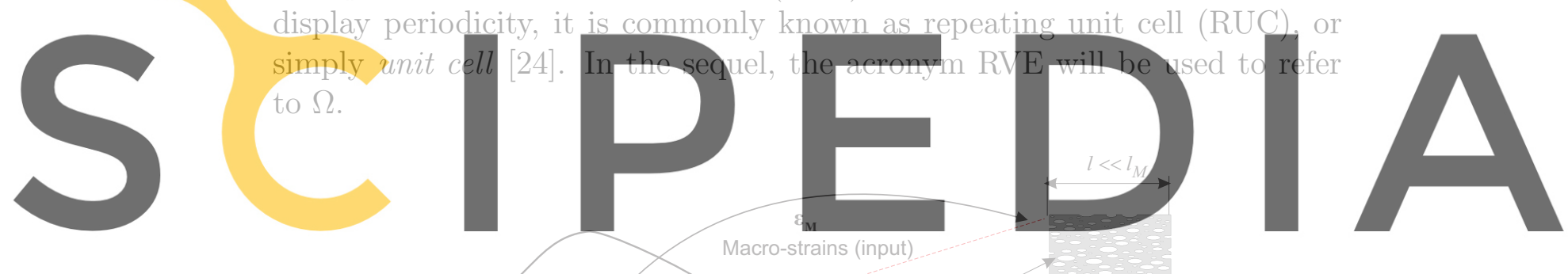

Register for free at https//Xww.scipedia.com to downtoad" the version without the watermark

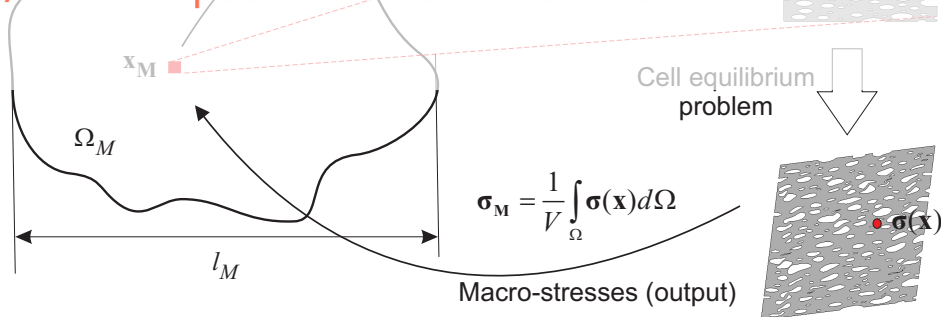

Figure 1: First-order homogenization.

In the homogenization approach adopted in this work - commonly known as first-order homogenization $[32,40]-$, the strain field $\boldsymbol{\epsilon}(\mathbf{x})$ at any point $\mathbf{x} \in \Omega$ is assumed to be decomposed into macroscopic and fluctuating contributions; under the hypothesis of infinitesimal deformations, this decomposition can be written as:

$$
\boldsymbol{\epsilon}(\mathbf{x})=\boldsymbol{\epsilon}_{M}+\nabla^{s} \boldsymbol{u}(\mathbf{x})
$$


Here, $\boldsymbol{\epsilon}_{\boldsymbol{M}}$ stands $^{3}$ for the macroscopic strain tensor (the input parameter in the problem) and $\nabla^{s} \boldsymbol{u}$ denotes the symmetric gradient of the displacement fluctuation field (this field is, in turn, the basic unknown of the problem).

Implicit in the scale separation assumption is the fact that fine-scale deformations only influence coarse-scale behavior through its volume average over the RVE. It can be shown (see, for instance, Ref. [22]) that this implies that the boundary conditions (BCs) prescribed on the RVE must be homogeneous (i.e., $\boldsymbol{A}_{0} \boldsymbol{u}=\mathbf{0}$ on $\partial \Omega, \boldsymbol{A}_{0}$ being a certain linear operator). The natural choice for a repeating unit cell is to employ periodic boundary conditions (See Refs. [9, 48] for more details on how to prescribe this type of $\mathrm{BCs}$ ). In statistically homogeneous micro-structures, by contrast, there is a certain latitude in the choice of boundary conditions (vanishing fluctuations, uniform tractions, quasi-periodic conditions ...). In the examples shown later, vanishing boundary conditions are used $(\boldsymbol{u}=0$ on $\partial \Omega)$.
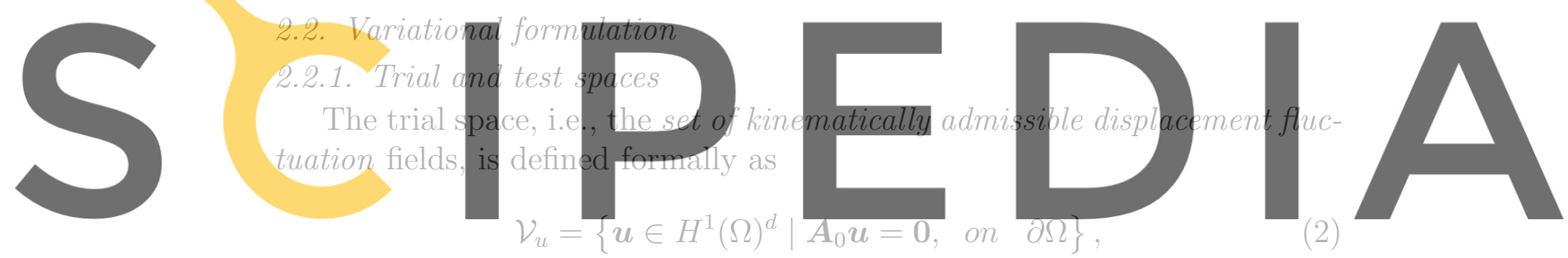

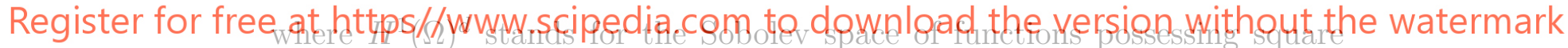

integrable derivatives over $\Omega$. Note that this set forms a vector space. Since

the test functions $\boldsymbol{\eta}$ appearing in the variational statement shown in the following are kinematically admissible variations $\left(\boldsymbol{\eta}:=\mathbf{u}-\mathbf{v}, \quad \mathbf{u}, \mathbf{v} \in \mathcal{V}_{\mathbf{u}}\right)$, $\mathcal{V}_{u}$ having structure of vector space implies that, in the RVE equilibrium problem, the spaces of trial and test functions coincide.

\subsubsection{Formal statement}

Consider a time discretization of the interval of interest $\left[t_{0}, t_{f}\right]=\bigcup_{n=1}^{n_{s t p}}\left[t_{n}, t_{n+1}\right]$. The current value of the microscopic stress tensor $\boldsymbol{\sigma}_{n+1}$ at each $\mathbf{x} \in \Omega$ is presumed to be entirely determined by, on the one hand, the current value of the

\footnotetext{
${ }^{3}$ Macroscopic variables will be identified by appending a subscript " $M$ ", while variables associated to the fine scale will be designated by bare symbols. For instance, we shall write $\boldsymbol{\epsilon}_{M}$ and $\boldsymbol{\epsilon}(\mathbf{x})$ to denote the macroscopic strain tensor and the fine-scale strain field, respectively.
} 
microscopic strain tensor $\boldsymbol{\epsilon}_{n+1}(\mathbf{x})=\boldsymbol{\epsilon}_{\boldsymbol{M}_{n+1}}+\nabla^{s} \boldsymbol{u}_{n+1}(\mathbf{x})$, and, on the other hand, a set of microscopic internal variables $\boldsymbol{\xi}_{n+1}$ - that encapsulate the history of microscopic deformations. The relationship between these variables is established by (phenomenological) rate constitutive equations; these equations may vary from point to point within the RVE (multiphase materials). Likewise, the considered RVE may contain also voids distributed all over the domain. The (incremental) RVE equilibrium problem at time $t_{n+1}$ can be stated as follows (see Ref. [22]): given the initial data $\left\{\boldsymbol{u}_{n}(\mathbf{x}), \boldsymbol{\epsilon}_{\boldsymbol{M}_{n}}, \boldsymbol{\xi}_{n}(\mathbf{x})\right\}$ and the prescribed macroscopic strain tensor $\epsilon_{M n+1}$, find $u_{n+1} \in \mathcal{\nu}_{u}$ such that

$$
\int_{\Omega} \nabla^{s} \boldsymbol{\eta}: \boldsymbol{\sigma}_{n+1}\left(\boldsymbol{\epsilon}_{M_{n+1}}+\nabla^{s} \boldsymbol{u}_{n+1}, \boldsymbol{\xi}_{n+1}\right) d \Omega=0,
$$

for all $\eta \in \mathcal{V}_{u}$. The actual output of interest in this fine-scale BVP is not the displacement fluctuation field per se, but rather the macroscopic stress tensor $\left.\sigma_{M}\right|_{n+1}$, which is defined as the volume average over the RVE of the
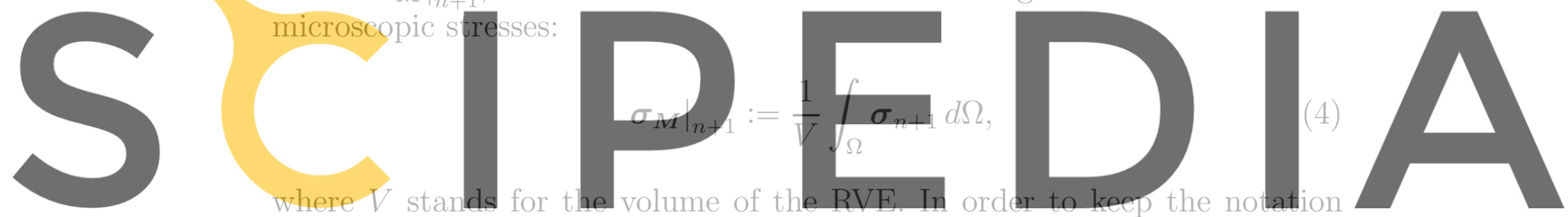

uncluttered, the superindex " $\mathrm{n}+1$ " will be hereafter dropped out and all

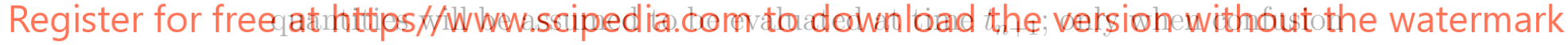

is apt to show up, the pertinent distinction will be introduced.

\subsection{Finite element formulation}

Let $\Omega=\bigcup_{n=1}^{n_{e}} \Omega^{e}$ be a finite element discretization of the RVE. It will be assumed that this discretization is fine enough to consider the exact and FE approximated solutions indistinguishable at the accuracy level of interest. Let $\left\{N_{1}(\mathbf{x}), N_{2}(\mathbf{x}) \ldots N_{n}(\mathbf{x})\right\}$ ( $n$ denotes the number of nodes of the discretization) be a set of shape functions associated to this discretization. Now we approximate $\boldsymbol{u} \in \mathcal{V}_{u}$ and $\boldsymbol{\eta} \in \mathcal{V}_{u}$ as

$$
\begin{gathered}
\boldsymbol{u}\left(\mathbf{x} ; \boldsymbol{\epsilon}_{\boldsymbol{M}}\right) \approx \boldsymbol{u}^{(h)}\left(\mathbf{x} ; \boldsymbol{\epsilon}_{\boldsymbol{M}}\right)=\sum_{I=1}^{n} N_{I}(\mathbf{x}) \boldsymbol{U}_{I}\left(\boldsymbol{\epsilon}_{\boldsymbol{M}}\right) \\
\boldsymbol{\eta}(\mathbf{x}) \approx \boldsymbol{\eta}^{(\boldsymbol{h})}(\mathbf{x})=\sum_{I=1}^{n} N_{I}(\mathbf{x}) \boldsymbol{\eta}_{I}
\end{gathered}
$$


where $\boldsymbol{U}_{I} \in \mathbb{R}^{d}$ and $\boldsymbol{\eta}_{I} \in \mathbb{R}^{d}(I=1,2 \ldots n)$ denote the nodal values of the displacement fluctuations and test functions, respectively. Inserting these approximations in Eq.(3), and exploiting the arbitrariness of coefficients $\boldsymbol{\eta}_{I}$ $(I=1,2 \ldots n)$, one arrives at the following set of discrete equilibrium equations (repeated indices implies summation):

$$
\int_{\Omega} \frac{\partial N_{I}}{\partial x_{j}} \boldsymbol{\sigma}_{i j}\left(\boldsymbol{\epsilon}_{\boldsymbol{M}}+\nabla^{s} \boldsymbol{u}^{(h)}, \boldsymbol{\xi}\right) d \Omega=0 \quad(i=1 \ldots d ; I=1 \ldots n) .
$$

Introducing Voigt's notation ${ }^{4}$, the above equation can be expressed in matrix format as:

$$
\int_{\Omega} \boldsymbol{B}^{T} \boldsymbol{\sigma}\left(\boldsymbol{\epsilon}_{\boldsymbol{M}}+\boldsymbol{B} \boldsymbol{U}, \boldsymbol{\xi}\right) d \Omega=\mathbf{0}
$$

As usual, numerical evaluation of the integral in Eq.(8) is carried out by Gaussian quadrature:

$$
\int_{\Omega} \boldsymbol{B}^{T} \boldsymbol{\sigma} d \Omega \approx \sum_{g=1}^{n_{g}} w_{g} \boldsymbol{B}^{T}\left(\mathbf{x}_{g}\right) \boldsymbol{\sigma}\left(\mathbf{x}_{g}, ;\right)=\mathbf{0} .
$$

Here, $n_{g}=\mathcal{O}(n)$ stands for the total number of Gauss points of the mesh; $w_{g}$ denotes the weight associated to the $g-t h$ Gauss point $\mathbf{x}_{g}$ (this weight includes both the quadrature weight itself and the corresponding Jacobian determinant.); and $\boldsymbol{B}\left(\mathbf{x}_{g}\right)$ and $\boldsymbol{\sigma}\left(\mathbf{x}_{g}, ;\right)$ stand for the B-matrix and the stress vector at Gauss point $\mathbf{x}_{g}$, respectively.

\section{Computation of reduced basis}

A basic, intuitive picture of the strategy for computing the reduced basis onto which to project the RVE equilibrium equation (3) was already given in the introductory section. In the following, we put the idea behind this strategy on a more rigorous footing. We begin by noting that, from a functional analysis standpoint, the term model reduction is conceptually akin

\footnotetext{
${ }^{4}$ Here, it is convenient to use the so-called modified Voigt's notation rather than the standard one. In the modified Voigt's notation, both stress $\boldsymbol{\sigma}$ and strain $\boldsymbol{\epsilon}$ tensors are represented as column vectors $(\{\boldsymbol{\sigma}\}$ and $\{\boldsymbol{\epsilon}\}$, respectively $)$ in which the shear components are multiplied by $\sqrt{2}$. The advantage of this notation over the conventional, engineering Voigt's notation is the equivalence between norms; viz., $\|\boldsymbol{\sigma}\|=\sqrt{\boldsymbol{\sigma}: \boldsymbol{\sigma}}=\|\{\boldsymbol{\sigma}\}\|=$ $\sqrt{\{\boldsymbol{\sigma}\}^{T}\{\boldsymbol{\sigma}\}}$. The reader is urged to consult [20] for further details on this notation.
} 
to the more common term model discretization, since both connote transitions from higher-dimensional to lower-dimensional solution spaces. Whereas model discretization is used to refer to the (classical) passage from the infinite dimensional space $\mathcal{V}_{u}$ to the finite element subspace $\mathcal{V}_{u}^{h} \subset \mathcal{V}_{u}$, model reduction denotes a transition from this finite dimensional space $\mathcal{V}_{u}^{h}$ to a significantly smaller manifold $\mathcal{V}_{u}^{*} \subset \mathcal{V}_{u}^{h}$-the reduced-order space. This latter transition is not carried out directly, but in two sequential steps, namely, sampling of the input parameter space and dimensionality reduction.

\subsection{Sampling of the input parameter space}

In constructing the finite element space of kinematically admissible functions $\mathcal{V}_{u}^{h}$, the only restrictions placed on the motion of the mesh nodes are those imposed at the boundaries. The finite element solution space, thus, does not presuppose any constraint on the motion of the interior nodes of the mesh.

However, in actuality, interior nodes cannot fluctuate freely, independently from each other, but they rather move according to deformational patterns dictated by the constitutive laws that govern the mechanical behavior of the distinct phases in the $\mathrm{RVE}^{5}$. This means that the solution of the finite element equilibrium equation (3) for given values of the macro-strain tensor $\boldsymbol{\epsilon}_{\boldsymbol{M}}$ actually lives in a smaller subspace $\mathcal{V}_{u}^{\epsilon} \subset \mathcal{V}_{u}^{h}$ (in the parlance of model reduction $[47,57], \mathcal{V}_{u}^{\epsilon}$ is the manifold induced by the parametric dependence of the BVP on the input variables).

Yet, in general, this subspace cannot be precisely determined; one has to be content to construct an approximation of it as the span of the displacement fluctuation solutions obtained for a judiciously chosen set of $n_{\text {hst }}$ input

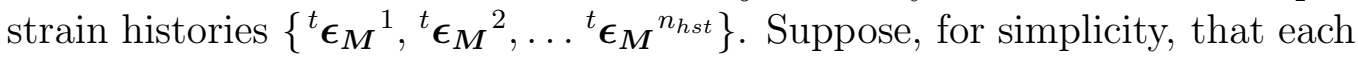
of these strain histories is discretized into equal number of steps $n_{s t p}$, and let

$$
\boldsymbol{u}^{k}(\mathbf{x})=\boldsymbol{u}\left(\mathbf{x} ;{ }^{t} \boldsymbol{\epsilon}_{\boldsymbol{M}_{j}}{ }^{i}\right), \quad k=(i-1) n_{h s t}+j
$$

denote the displacement fluctuation solution at the $j-t h$ time step of the $i-t h$ strain history $\left(i=1,2 \ldots n_{h s t}, j=1,2 \ldots n_{s t p}\right)$. The approximating space for $\mathcal{V}_{u}^{\epsilon}$, henceforth called the snapshots space, is then defined as:

\footnotetext{
${ }^{5}$ As noted by Lubliner [45], constitutive laws can be regarded as internal restrictions on the kinds of deformation a body can suffer
} 


$$
\mathcal{V}_{u}^{s n p}=\operatorname{span}\left\{\boldsymbol{u}^{1}(\mathbf{x}), \boldsymbol{u}^{2}(\mathbf{x}), \ldots \boldsymbol{u}^{n_{s n p}}(\mathbf{x})\right\} \subseteq \mathcal{V}_{u}^{\epsilon},
$$

$n_{s n p}=n_{s t p} n_{h s t}$ being the total number of snapshots. The matrix containing, in columns, the nodal values of these displacement fluctuations solutions:

$$
\boldsymbol{X}_{u}=\left[\begin{array}{llll}
\boldsymbol{U}^{1} & \boldsymbol{U}^{2} & \cdots & \boldsymbol{U}^{n_{s n p}}
\end{array}\right] \in \mathbb{R}^{n \cdot d \times n_{s n p}}
$$

will correspondingly be termed the (displacement fluctuations) snapshot matrix.

\subsection{Dimensionality reduction}

The next and definitive step in the transition from the high-dimensional finite element space $\mathcal{V}_{u}^{h}$ to the desired reduced-order space $\mathcal{V}_{u}^{*}$-in which the fine-scale BVP is to be finally posed - is the dimensionality reduction process, in which, as pointed out in the introductory section, the dominant deformational patterns of the RVE response are identified and unveiled by washing out the "inessentials". To accomplish this central task, we employ here a partitioned version of the Proper Orthogonal Decomposition ${ }^{6}$.

\subsubsection{Elastic/Inelastic reduced basis functions}

The Proper Orthogonal Decomposition is nothing but a multidimensional data fitting procedure intended to obtain a sequence of orthogonal basis functions whose span best approximate the space of snapshots. As such, the POD is a purely data-driven process - it is "agnostic" to the physical origin of the data. For instance, for POD basis construction purposes, it is completely immaterial whether a given snapshot corresponds to a purely linear elastic solution or to a solution well into the inelastic regime. The task of discriminating which features of the RVE response are essential and which are not is exclusively guided by statistical considerations: if the elastic response happens to be poorly represented within the snapshot ensemble, the POD may regard as unimportant the contribution of these snapshots, and, as a consequence, the basis functions with largest associated singular values -i.e., the essential modes - would hardly contain any information of this range. To accurately replicate the apparently trivial linear elastic behavior, thus, one may be forced to take a relatively large number of basis functions, and this

\footnotetext{
${ }^{6}$ See Appendix A for a brief description of the POD.
} 
may translate into a significant increase in the overall online computational cost. This fact certainly places the POD-based reduced basis approach at a competitive disadvantage compared with semi-analytical homogenization approaches such as the Nonlinear Transformation Field Analysis [50], which do capture exactly (and effortlessly) the linear elastic response of the RVE.

To eliminate this shortcoming, we propose here a slightly different strategy for constructing the reduced basis. The essence of the proposal is to partition the space of snapshots $\mathcal{V}_{u}^{s n p}$ into elastic $\left(\mathcal{V}_{u, \text { el }}^{\text {snp }}\right)$ and inelastic $\left(\mathcal{V}_{u, \text { inel }}^{\text {snp }}\right)$ subspaces:

$$
\mathcal{V}_{u}^{s n p}=\mathcal{V}_{u, e l}^{s n p} \oplus \mathcal{V}_{u, i n e l}^{s n p},
$$

( $\oplus$ symbolizes direct sum of subspaces [55]) and then obtain the reduced basis as the union of the bases for both subspaces. Below, we describe this strategy more in detail.

The first step is to determine an orthogonal basis for $\mathcal{V}_{u, e l}^{s n p}$. One can do this by simply performing $m_{e}$ independent, linear elastic finite element analysis of the RVE $\left(m_{e}=6\right.$ for $3 \mathrm{D}$ problems ${ }^{7}$, and $m_{e}=3$ for plane strain), and then orthonormalizing the resulting displacement fluctuation fields. These $m_{e}$ elastic modes will be considered as the first $m_{e}$ basis functions of the reduced basis:

$$
\operatorname{span}\left\{\boldsymbol{\Phi}_{1}, \boldsymbol{\Phi}_{2}, \ldots, \boldsymbol{\Phi}_{m_{e}}\right\}=\mathcal{V}_{u, e l}^{s n p} .
$$

Once we have at our disposal this set of elastic basis functions, we compute the (orthogonal) projection of each snapshot $\boldsymbol{u}^{k}$ onto the orthogonal complement of $\mathcal{V}_{u, e l}^{\text {snp }}$ (which is precisely the aforementioned inelastic space $\mathcal{V}_{u, \text { inel }}^{\text {snp }}$ ):

$$
\boldsymbol{u}_{\text {inel }}^{k}:=\boldsymbol{u}^{k}-\sum_{i=1}^{m_{e}}\left\langle\boldsymbol{\Phi}_{i}, \boldsymbol{u}^{k}\right\rangle_{L_{2}(\Omega)} \boldsymbol{\Phi}_{i}, \quad k=1,2 \ldots n_{\text {snp }} .
$$

It is now on this ensemble of inelastic snapshots $\left\{\boldsymbol{u}_{i n e l}^{k}\right\}_{k=1}^{n_{s n p}}$ that the previously described POD is applied to obtain the remaining $n_{u}-m_{e}$ basis functions. Thus, we finally have:

$$
\mathcal{V}_{u}^{*}=\mathcal{V}_{u, e l}^{\text {snp }} \oplus \mathcal{V}_{u, \text { inel }}^{\text {snp }}=\operatorname{span}\{\overbrace{\boldsymbol{\Phi}_{1}, \boldsymbol{\Phi}_{2}, \ldots, \boldsymbol{\Phi}_{6}}^{\text {Elastic modes }}, \overbrace{\boldsymbol{\Phi}_{7}, \ldots, \boldsymbol{\Phi}_{n_{u}}}^{\text {"Essential" Inelastic modes }}\} .
$$

\footnotetext{
${ }^{7}$ Strictly speaking, the proposed decomposition is only valid for materials governed by rate-independent constitutive equations.
} 
for 3D problems, and

$$
\mathcal{V}_{u}^{*}=\operatorname{span}\{\overbrace{\boldsymbol{\Phi}_{1}, \boldsymbol{\Phi}_{2}, \boldsymbol{\Phi}_{3}}^{\text {Elastic modes }}, \quad \overbrace{\boldsymbol{\Phi}_{4}, \ldots, \boldsymbol{\Phi}_{n_{u}}}^{\text {"Essential" inelastic modes }}\} .
$$

for plane strain. In placing the $m_{e}$ elastic modes within the first $m_{e}$ positions, the reduced-order model is guaranteed to deliver linear elastic solutions with the same accuracy as the underlying (full-order) finite element model (obviously, provided that $n_{u} \geq m_{e}$ ).

Further details concerning the numerical implementation of this apparently novel - to the best of the authors' knowledge- basis construction strategy can be found in Appendix B.

\section{Galerkin projection onto the reduced subspace}

We now seek to pose the boundary-value problem represented by Eq.(3) in the reduced-order space $\mathcal{V}_{u}^{*} \subseteq \mathcal{V}_{u}^{h}$ spanned by the basis functions $\left\{\boldsymbol{\Phi}_{1}, \boldsymbol{\Phi}_{2}, \ldots, \boldsymbol{\Phi}_{n_{u}}\right\}$. To this end, we approximate both test $\boldsymbol{\eta} \in \mathcal{V}_{u}$ and trial $\boldsymbol{u} \in \mathcal{V}_{u}$ functions by the following linear expansions:

$$
\begin{gathered}
\boldsymbol{u}\left(\mathbf{x} ; \boldsymbol{\epsilon}_{\boldsymbol{M}}\right) \approx \boldsymbol{u}^{*}\left(\mathbf{x} ; \boldsymbol{\epsilon}_{\boldsymbol{M}}\right)=\sum_{i=1}^{n_{u}} \boldsymbol{\Phi}_{i}(\mathbf{x}) U_{i}^{*}\left(\boldsymbol{\epsilon}_{\boldsymbol{M}}\right) \\
\boldsymbol{\eta}(\mathbf{x}) \approx \boldsymbol{\eta}^{*}(\mathbf{x})=\sum_{i=1}^{n_{u}} \boldsymbol{\Phi}_{i}(\mathbf{x}) \eta_{i}^{*}
\end{gathered}
$$

$\boldsymbol{u}^{*}(\mathbf{x})$ and $\boldsymbol{\eta}^{*}(\mathbf{x})$ being the low-dimensional approximations of trial and test functions, respectively (hereafter, asterisked symbols will be used to denote low-dimensional approximations of the associated variables). Inserting Eqs. (18) and (19) into Eq.(3), and exploiting the arbitrariness of coefficients $\eta_{i}^{*}$ $\left(i=1,2 \ldots n_{u}\right)$, we arrive at the following set of $n_{u}$ equilibrium equations:

$$
\int_{\Omega} \nabla^{s} \boldsymbol{\Phi}_{i}(\mathbf{x}): \boldsymbol{\sigma}\left(\mathbf{x} ; \boldsymbol{\epsilon}_{\boldsymbol{M}}+\nabla^{s} \boldsymbol{u}^{*}, \boldsymbol{\xi}\right) d \Omega=0, \quad i=1,2 \ldots n_{u} .
$$

Expressing now the reduced basis functions in the above equation in terms of finite element shape functions (through expression $\boldsymbol{\Phi}_{i}(\mathbf{x})=\sum_{I=1}^{n} N_{I}(\mathbf{x}) \boldsymbol{\Phi}_{I i}$ ), we get (in Voigt's notation):

$$
\int_{\Omega} \boldsymbol{B}_{i}^{* T}(\mathbf{x}) \boldsymbol{\sigma}\left(\mathbf{x} ; \boldsymbol{\epsilon}_{\boldsymbol{M}}+\boldsymbol{B}^{*} \boldsymbol{U}^{*}, \boldsymbol{\xi}\right) d \Omega=\mathbf{0}, \quad i=1,2 \ldots n_{u}
$$


or more compactly:

$$
\int_{\Omega} \boldsymbol{B}^{* T}(\mathbf{x}) \boldsymbol{\sigma}\left(\mathbf{x} ; \boldsymbol{\epsilon}_{\boldsymbol{M}}+\boldsymbol{B}^{*} \boldsymbol{U}^{*}, \boldsymbol{\xi}\right) d \Omega=\mathbf{0} .
$$

Here, $\boldsymbol{U}^{*}=\left[\begin{array}{llll}U_{1}^{*} & U_{2}^{*} & \cdots & U_{n_{u}}^{*}\end{array}\right]^{T} \in \mathbb{R}^{n_{u}}$ denotes the vector containing the reduced displacement fluctuations - the basic unknowns of the reduced-order problem - and $\boldsymbol{B}^{*}: \Omega \rightarrow \mathbb{R}^{s \times n_{u}}$ stands for the reduced "B-matrix", defined as:

$$
\boldsymbol{B}^{*}(\mathbf{x}):=\boldsymbol{B}(\mathbf{x}) \Phi
$$

This matrix connects the gradient of the displacement fluctuation field with the vector of reduced displacement fluctuations:

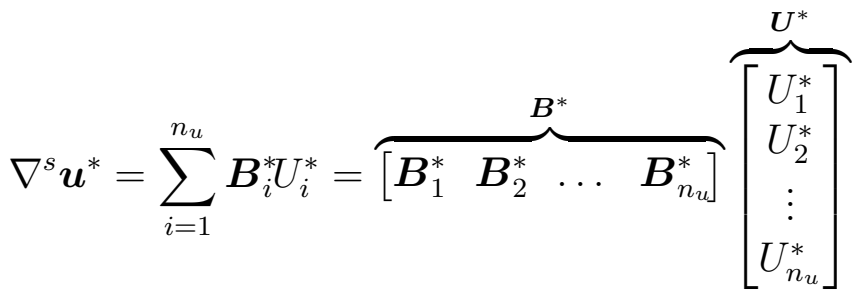

$$
\begin{aligned}
& =\boldsymbol{B}^{*} \boldsymbol{U}^{*}=\boldsymbol{B} \Phi \boldsymbol{U}^{*} .
\end{aligned}
$$

For implementational purposes, it is more expedient to express Eq.(23) in terms of elemental $B$-matrices. To this end, we write:

$$
\boldsymbol{B}(\mathbf{x})=\left\{\begin{array}{l}
\boldsymbol{B}^{e}(\mathbf{x}), \quad \text { if } \mathbf{x} \in \Omega^{e} \\
\mathbf{0}, \quad \text { otherwise }
\end{array}\right.
$$

where $\boldsymbol{B}^{e} \in \mathbb{R}^{s \times d \cdot \bar{n}_{e}}$ denotes the local $B$-matrix of element $\Omega^{e}\left(\bar{n}_{e}\right.$, in turn, is the number of nodes in $\left.\Omega^{e}\right)$. Thus,

$$
\boldsymbol{B}^{*}(\mathrm{x})=\boldsymbol{B}(\mathrm{x}) \boldsymbol{\Phi}=\boldsymbol{B}^{e}(\mathrm{x}) \Phi^{e} .
$$

In the above equation, $\boldsymbol{\Phi}^{e} \in \mathbb{R}^{d \bar{n}_{e} \times n_{u}}$ represents the block matrix of $\boldsymbol{\Phi}$ corresponding to the $\bar{n}_{e}$ nodes of finite element $\Omega^{e}\left(e=1,2 \ldots n_{e}\right)$.

\section{Stress approximation space}

To arrive at an efficient, mesh-size independent integration scheme, two crucial questions have to be addressed, namely, the determination of the 
vector space (hereafter denoted by $\mathcal{V}_{\sigma}^{a p r}$ ) in which the low-dimensional approximation of the stress field ${ }^{8}$ should lie in order to obtain an accurate and at the same time well-posed ROM; and the calculation of the optimal location of the sampling or integration points. Attention here and in the next section is confined to the aspect related to the stress approximation space; the issue related to the selection of sampling points, on the other hand, is examined in Section 7.

\subsection{The reduced-order subspace of statically admissible stresses $\left(\mathcal{V}_{\sigma}^{*}\right)$}

At first sight, the problem of constructing a $\mathcal{O}\left(n_{u}\right)$-dimensional representation of the stress field seems quite similar to the problem addressed in Section 3 concerning the reduced basis for the displacement fluctuations: we have to find a set of orthogonal basis functions $\left\{\boldsymbol{\Psi}_{1}(\mathbf{x}), \boldsymbol{\Psi}_{2}(\mathbf{x}) \ldots \boldsymbol{\Psi}_{n_{\sigma}}(\mathbf{x})\right\}$ $\left(n_{\sigma}=\mathcal{O}\left(n_{u}\right)\right)$ such that its span accurately approximates the set of all possible stress solutions - that is, the set of all statically admissible stresses. Accordingly, following the procedure described in Section 3, we first compute finite element, stress distributions over the RVE for representative macrostrain histories ${ }^{9}$. Then, the elastic/inelastic dimensionality reduction process set forth in Section 3.2.1 is applied to the resulting ensemble of stress solutions $\left\{\boldsymbol{\sigma}^{1}(\mathbf{x}), \boldsymbol{\sigma}^{2}(\mathbf{x}) \ldots \boldsymbol{\sigma}^{n_{s n p}}(\mathbf{x})\right\}$, in order to identify both the elastic and the essential inelastic stress modes. The space spanned by these modes will be denoted hereafter by $\mathcal{V}_{\sigma}^{*}$ and termed the reduced-order subspace of statically admissible stresses:

$$
\mathcal{V}_{\sigma}^{*}=\operatorname{span}\{\overbrace{\boldsymbol{\Psi}_{1}(\mathbf{x}), \boldsymbol{\Psi}_{2}(\mathbf{x}), \ldots, \boldsymbol{\Psi}_{m_{e}}(\mathbf{x})}^{\text {Elastic stress modes }}, \overbrace{\boldsymbol{\Psi}_{m_{e}+1}(\mathbf{x}), \boldsymbol{\Psi}_{m_{e}+2}(\mathbf{x}), \ldots, \boldsymbol{\Psi}_{n_{\sigma}}(\mathbf{x})}^{\text {"Essential", inelastic stress modes }}\} .
$$

\footnotetext{
${ }^{8}$ It was mentioned in the introductory section that the central idea of efficient interpolatory approaches for numerical integration of reduced-order BVPs is to replace the nonaffine term in the the integrand by low-dimensional interpolants. In our case, a glance at the reduced-order equilibrium equation (21) readily reveals that such "offending", nonaffine term is the stress field - the reduced $B$-matrix $\boldsymbol{B}^{*}=\boldsymbol{B}^{*}(\mathbf{x})$ is independent of the input parameter $\boldsymbol{\epsilon}_{\boldsymbol{M}}$ and hence need not be subject to approximation.

${ }^{9}$ The most practical and somehow consistent choice regarding these strain trajectories is to use the same as in the computation of the displacement fluctuations snapshots
} 


\subsection{Ill-posedness of the HP-ROM}

Let us now try to construct the low-dimensional approximation of the stress field, denoted by ${ }^{10} \boldsymbol{\sigma}^{*}$, as a linear combination of the above described stress reduced basis - hence making $\mathcal{V}_{\sigma}^{a p r}=\mathcal{V}_{\sigma}^{*}$-; i.e.,

$$
\boldsymbol{\sigma}\left(\mathbf{x} ; \boldsymbol{\epsilon}_{\boldsymbol{M}}, \boldsymbol{U}^{*}\right) \approx \boldsymbol{\sigma}^{*}\left(\mathbf{x} ; \boldsymbol{\epsilon}_{\boldsymbol{M}}, \boldsymbol{U}^{*}\right)=\sum_{i=1}^{n_{\sigma}} \boldsymbol{\Psi}_{i}(\mathbf{x}) c_{i}\left(\boldsymbol{\epsilon}_{\boldsymbol{M}}, \boldsymbol{U}^{*}\right)
$$

where $c_{i} \in \mathbb{R}\left(i=1,2 \ldots n_{\sigma}\right)$. This strategy of approximating the offending, nonaffine term in the BVP by a linear combination of pre-computed basis functions - obtained, in turn, from samples of the nonaffine term evaluated at the solution - has been successfully applied by several authors, with no apparent - or at least not reported - computational pitfalls, to a wide gamut of problems: nonlinear monotonic elliptic and nonlinear parabolic BPVs [46, 33], nonlinear miscible viscous fingering in porous media $[17,18]$, uncertainty quantification in inverse problems [31], and nonlinear heat conduction problems $[5,6]$, to cite but a few.

However, a closer examination of the the RVE equilibrium problem reveals that, in this case, this "standard" strategy proves completely fruitless, for it leads to patently ill-posed reduced-order equations. To show this, let us first substitute approximation (28) into Eq.(21):

$$
\begin{aligned}
& \int_{\Omega} \boldsymbol{B}^{* T}(\mathbf{x}) \boldsymbol{\sigma}\left(\mathbf{x} ; \boldsymbol{\epsilon}_{\boldsymbol{M}}, \boldsymbol{U}^{*}\right) d \Omega \approx \int_{\Omega} \boldsymbol{B}^{* T}(\mathbf{x}) \boldsymbol{\sigma}^{*}\left(\mathbf{x} ; \boldsymbol{\epsilon}_{\boldsymbol{M}}, \boldsymbol{U}^{*}\right)= \\
& \sum_{i=1}^{n_{\sigma}}\left(\int_{\Omega} \boldsymbol{B}^{* T}(\mathbf{x}) \boldsymbol{\Psi}_{i}(\mathbf{x}) d \Omega\right) c_{i}\left(\boldsymbol{\epsilon}_{\boldsymbol{M}}, \boldsymbol{U}^{*}\right)=\mathbf{0} .
\end{aligned}
$$

By virtue of Eq.(23), the bracketed integral in the preceding equation can be rephrased as:

$$
\int_{\Omega} \boldsymbol{B}^{* T}(\mathbf{x}) \boldsymbol{\Psi}_{i}(\mathbf{x}) d \Omega=\boldsymbol{\Phi}^{T}\left(\int_{\Omega} \boldsymbol{B}^{T}(\mathbf{x}) \boldsymbol{\Psi}_{i}(\mathbf{x}) d \Omega\right), \quad i=1,2 \ldots n_{\sigma} .
$$

Each basis function $\boldsymbol{\Psi}_{i}(\mathbf{x})\left(i=1,2 \ldots n_{\sigma}\right)$ is, by construction, a linear combination of the stress snapshots collected during the offline, finite element

\footnotetext{
${ }^{10}$ Notice that, in keeping with the notational convention introduced in Section 4 , the low-dimensional approximation of the stress field is represented by attaching an asterisk to the stress symbol.
} 
analysis; thus, we can write $\boldsymbol{\Psi}_{i}=\sum_{j=1}^{n_{s n p}} \beta_{i j} \boldsymbol{\sigma}^{j}\left(i=1,2 \ldots n_{\sigma}\right), \beta_{i j} \in \mathbb{R}$ being the corresponding coefficients in the linear combination. Inserting the above equation into Eq.(30) and considering that $\boldsymbol{\sigma}^{j}\left(j=1,2 \ldots n_{\text {snp }}\right)$ are finite element stress solutions - and therefore fulfill the finite element equilibrium equation (8) - , we finally arrive at:

$$
\boldsymbol{\Phi}^{T} \sum_{j=1}^{n_{s n p}} \beta_{i j} \overbrace{\left(\int_{\Omega} \boldsymbol{B}^{T} \boldsymbol{\sigma}^{j} d \Omega\right)}^{=0}=\mathbf{0}, \quad i=1,2 \ldots n_{\sigma},
$$

that is, the integral (30) appearing in the equilibrium equation (29), and hence, the left-hand side of the equation itself, vanishes identically regardless of the value of the modal coefficients $c_{i} \in \mathbb{R}\left(i=1,2 \ldots n_{\sigma}\right)$, and therefore, regardless of the value of the reduced displacement fluctuations $\boldsymbol{U}^{*}$ - hence the ill-posedness.

\subsection{Proposed remedy: the expanded space approach}

It is clear from the foregoing discussion that the root cause of the illposedness lies in the fact that the set of all admissible stress fields $\left(\mathcal{V}_{\sigma}\right)$ forms a vector space, and, consequently, the POD stress modes $\boldsymbol{\Psi}_{i} \in \mathcal{V}_{\sigma}$ $\left(i=1,2 \ldots n_{\sigma}\right)$ - and any linear combination of them - turn out to be selfequilibrated fields. Thus, for the reduced-order problem to be well-posed, the approximation space $\mathcal{V}_{\sigma}^{a p r}$ cannot be only formed by statically admissible stresses, but it must also include statically inadmissible fields -i.e. stress functions that do not satisfy the reduced-order equilibrium equation (21).

One plausible route for determining a low-dimensional approximation space that embraces both statically admissible and statically inadmissible stresses might be to collect, during the offline finite element calculations, not only converged stresses, but also the unconverged ones -i.e., those generated during the corresponding iterative algorithm-, and then perform the POD-based dimensionality reduction over the whole ensemble of snapshots. In the present work, however, we pursue an approach that precludes the necessity of undertaking this computationally laborious and in some aspects objectionable - there is no guarantee that the span of selected, unconverged stress snapshots covers the entire space of statically inadmissible stresses process. The idea behind the employed approach was originally conceived, but not fully developed, by the authors in a recent monograph [35]. Here, the theory underlying such an idea is further elaborated and cast into the formalisms of functional analysis. 


\subsubsection{Continuum formulation}

To originate our considerations from a general standpoint, it proves convenient first to rephrase the left-hand side of the reduced-order equilibrium equation Eq.(21) as the action of a certain linear operator $\boldsymbol{G}: L_{2}(\Omega)^{s} \rightarrow \mathbb{R}^{n_{u}}$ on the stress field over the RVE:

$$
\int_{\Omega} \boldsymbol{B}_{i}^{* T} \boldsymbol{\sigma} d \Omega=\left\langle\boldsymbol{B}_{i}^{*}, \boldsymbol{\sigma}\right\rangle_{L_{2}(\Omega)}=(\boldsymbol{G}[\boldsymbol{\sigma}])_{i} \quad i=1,2 \ldots n_{u} .
$$

Invoking now the orthogonal decomposition of $L_{2}(\Omega)^{s}$ induced by this operator, one obtains:

$$
L_{2}(\Omega)^{s}=\mathcal{N}(\boldsymbol{G}) \oplus \operatorname{span}\left\{\boldsymbol{B}_{i}^{*}\right\}_{i=1}^{n_{u}},
$$

where $\mathcal{N}(\boldsymbol{G})$ stands for the nullspace of $\boldsymbol{G}$. Since the RVE equilibrium equation has a vanishing right-hand side term, it follows that $\mathcal{N}(\boldsymbol{G})$ is actually the space of statically admissible stress fields. Its orthogonal complement, $\operatorname{span}\left\{\boldsymbol{B}_{i}^{*}\right\}_{i=1}^{n_{u}}$, can be therefore construed as the abovementioned space of statically inadmissible stresses. The key fact here is that such a space is inherently $n_{u}$-dimensional and, thus, there is no need to perform any dimensionality reduction whatsoever over unconverged snapshots to arrive at the desired basis: the strain-displacement functions $\left\{\boldsymbol{B}_{1}^{*}, \boldsymbol{B}_{2}^{*} \ldots \boldsymbol{B}_{n_{u}}^{*}\right\}$ themselves are linearly independent (albeit not orthogonal) and can thereby serve this very purpose.

According to the preceding decomposition, any $\boldsymbol{\sigma} \in L_{2}(\Omega)^{s}$ can be resolved as (see Figure 2):

$$
\boldsymbol{\sigma}=\boldsymbol{\sigma}^{a d}+\boldsymbol{\sigma}^{i n}, \quad \text { with }\left\langle\boldsymbol{\sigma}^{a d}, \boldsymbol{\sigma}^{i n}\right\rangle_{L_{2}(\Omega)}=0,
$$

where $\boldsymbol{\sigma}^{a d} \in \mathcal{N}(\boldsymbol{G})$ and $\boldsymbol{\sigma}^{i n} \in \operatorname{span}\left\{\boldsymbol{B}_{i}^{*}\right\}_{i=1}^{n_{u}}$ stand for the statically admissible and statically inadmissible components of $\boldsymbol{\sigma}$, respectively. Following the standard approach, the statically admissible component $\boldsymbol{\sigma}^{a d}$-i.e., the stress solution we wish to calculate for a given input $\boldsymbol{\epsilon}_{\boldsymbol{M}}$ - is forced to lie in the span of the POD modes $\boldsymbol{\Psi}_{i}\left(i=1,2 \ldots n_{\sigma}\right)$ obtained from converged snapshots:

$$
\boldsymbol{\sigma}^{a d} \approx \boldsymbol{\sigma}^{*}=\sum_{i=1}^{n_{\sigma}} \boldsymbol{\Psi}_{i} c_{i}^{a d},
$$

$c_{i}^{a d} \in \mathbb{R}\left(i=1,2 \ldots n_{\sigma}\right)$ being the corresponding modal coefficients. The nonequilibrated component $\boldsymbol{\sigma}^{i n}$, on the other hand, resides naturally in the span 
of the reduced strain-displacement functions, so we can directly write-i.e., without introducing further approximations-

$$
\boldsymbol{\sigma}^{i n}=\sum_{i=1}^{n_{u}} \boldsymbol{B}_{i}^{*} c_{i}^{i n}
$$

with $c_{i}^{i n} \in \mathbb{R}\left(i=1,2 \ldots n_{u}\right)$. The low-dimensional approximation required in the proposed integration method, denoted in what follows by $\boldsymbol{\sigma}^{e x *}$ (the appended superscript "ex" means "stress approximated in the expanded space"), is finally obtained as the sum of Eq.(35) and Eq.(36) :

$$
\boldsymbol{\sigma}^{e x *}=\sum_{i=1}^{n_{\sigma}} \boldsymbol{\Psi}_{i} c_{i}^{a d}+\sum_{j=1}^{n_{u}} \boldsymbol{B}_{j}^{*} c_{j}^{i n} .
$$

Substituting the above approximation into the equilibrium equation, one

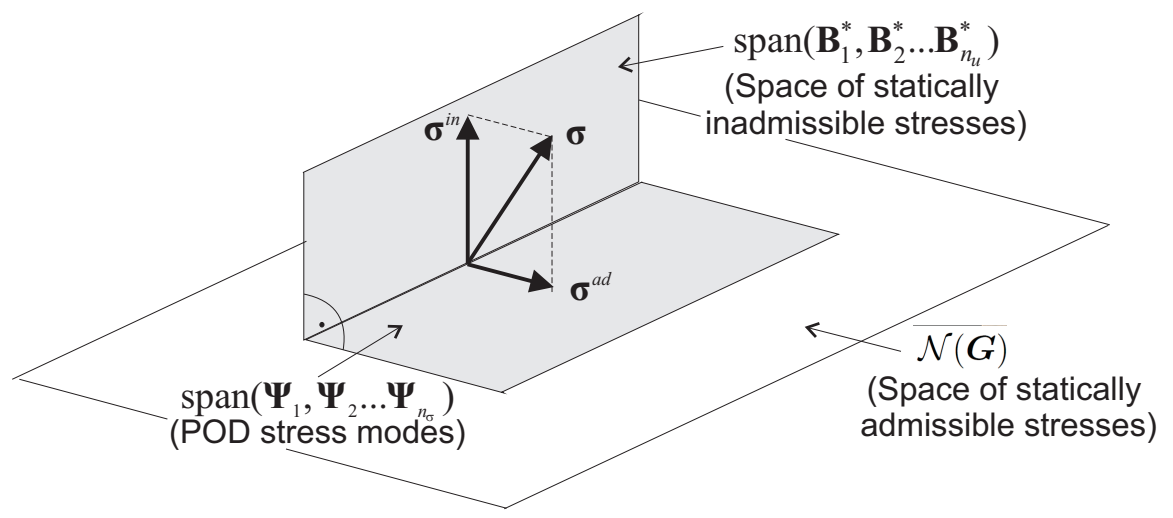

Figure 2: Expanded space approach. The stress approximation space is expanded so that it embraces, not only the span of the stress POD modes, but also the span of the reduced strain-displacement functions $\left\{\boldsymbol{B}_{1}^{*}, \boldsymbol{B}_{2}^{*} \ldots \boldsymbol{B}_{n_{u}}^{*}\right\}$. The reduced-order RVE equilibrium problem boils down to find the reduced displacement fluctuations vector $\boldsymbol{U}^{*}$ that makes the non-equilibrated component $\boldsymbol{\sigma}^{\text {in }}$ to vanish $\left(\boldsymbol{\sigma}^{\text {in }}\left(\boldsymbol{U}^{*}, \boldsymbol{\epsilon}_{\boldsymbol{M}}\right)=\mathbf{0}\right)$.

gets:

$$
\int_{\Omega} \boldsymbol{B}_{i}^{* T} \boldsymbol{\sigma}^{e x *}=\sum_{j=1}^{n_{u}}\left(\int_{\Omega} \boldsymbol{B}_{i}^{* T} \boldsymbol{B}_{j}^{*} d \Omega\right) c_{j}^{i n}=\mathbf{0}, \quad i=1,2 \ldots n_{u} .
$$

Since $\left\{\boldsymbol{B}_{1}^{*}, \boldsymbol{B}_{2}^{*} \ldots \boldsymbol{B}_{n_{u}}^{*}\right\}$ are linearly independent functions, it becomes immediately clear that the above equations holds only if:

$$
c_{j}^{i n}\left(\boldsymbol{\epsilon}_{\boldsymbol{M}}, \boldsymbol{U}^{*}\right)=0, \quad j=1,2 \ldots n_{u},
$$


i.e., if the $n_{u}$ coefficients multiplying $\boldsymbol{B}_{i}^{*} \in L_{2}(\Omega)^{s}\left(i=1,2 \ldots n_{u}\right)$ are identically zero. In adopting the proposed integration approach, thus, the reducedorder RVE equilibrium problem (21) is transformed into the problem of finding, for a given input macroscopic strain tensor $\boldsymbol{\epsilon}_{\boldsymbol{M}}$, the reduced displacement fluctuations vector $\boldsymbol{U}^{*} \in \mathbb{R}^{n_{u}}$ that makes the non-equilibrated component $\boldsymbol{\sigma}^{\text {in }}$ (defined in Eq.(36)) to vanish.

In a nutshell, the ill-posedness exhibited by the discrete problem when adopting the standard approach of using only POD modes is eliminated by expanding the stress approximation space so that it embraces also the span of the reduced strain-displacement functions (or strain modes ${ }^{11}$ ) $\boldsymbol{B}_{i}^{*} \in L_{2}(\Omega)^{s}$ $\left(i=1,2 \ldots n_{u}\right)$ :

$$
\mathcal{V}_{\sigma}^{a p r}=\mathcal{V}_{\sigma}^{*} \oplus \operatorname{span}\left\{\boldsymbol{B}_{i}^{*}\right\}_{i=1}^{n_{u}}=\operatorname{span}\{\overbrace{\boldsymbol{\Psi}_{1}, \boldsymbol{\Psi}_{2} \ldots \boldsymbol{\Psi}_{n_{\sigma}}}^{n_{\sigma} \text { stress modes }} \overbrace{\boldsymbol{B}_{1}^{*}, \boldsymbol{B}_{2}^{*} \ldots \boldsymbol{B}_{n_{u}}^{*}}^{n_{u} \text { strain modes }}\}
$$

\subsubsection{Discrete formulation}

In typical finite element implementations, both stresses and gradients of shape functions are only calculated and stored at the Gauss points of the underlying spatial discretization. For practical reasons, thus, it proves imperative to reformulate the above explained expanded space strategy and treat both magnitudes as spatially discrete variables, defined only at such Gauss points.

The discrete counterparts of the continuously defined fields $\boldsymbol{\sigma} \in L_{2}(\Omega)^{s}$ and $\boldsymbol{B}_{i}^{*} \in L_{2}(\Omega)^{s}\left(i=1,2 \ldots n_{u}\right)$ will be denoted by $\mathcal{S} \in \mathbb{R}^{n_{g} \cdot s}$ and $\mathcal{B}^{*}=$ $\left[\begin{array}{llll}\mathcal{B}_{1}^{*} & \mathcal{B}_{2}^{*} & \cdots & \mathcal{B}_{n_{u}}^{*}\end{array}\right] \in \mathbb{R}^{n_{g} \cdot s \times n_{u}}$, and termed the global stress vector, and the global matrix of strain modes, respectively. The global stress vector $\mathcal{S}$ is constructed by stacking the stress vectors $\boldsymbol{\sigma}\left(\mathbf{x}_{g} ; \cdot\right) \in \mathbb{R}^{s}\left(g=1,2 \ldots n_{g}\right)$ at the Gauss points of the finite element grid into a single column vector:

$$
\mathcal{S}:=\left[\begin{array}{llll}
\boldsymbol{\sigma}^{T}\left(\mathbf{x}_{1} ; \cdot\right) & \boldsymbol{\sigma}^{T}\left(\mathbf{x}_{2} ; \cdot\right) & \cdots & \boldsymbol{\sigma}^{T}\left(\mathbf{x}_{n_{g}} ; \cdot\right)
\end{array}\right]^{T} .
$$

Similarly, the global matrix of strain modes $\mathcal{B}^{*}$ is constructed as:

$$
\mathcal{B}^{*}:=\left[\begin{array}{llll}
\boldsymbol{B}^{* T}\left(\mathbf{x}_{1}\right) & \boldsymbol{B}^{* T}\left(\mathbf{x}_{2}\right) & \cdots & \boldsymbol{B}^{* T}\left(\mathbf{x}_{n_{g}}\right)
\end{array}\right]^{T} .
$$

\footnotetext{
${ }^{11}$ Indeed, functions $\boldsymbol{B}_{i}^{*} \in L_{2}(\Omega)^{s}\left(i=1,2 \ldots n_{u}\right)$ can be viewed as fluctuating strain modes, since they are the symmetric gradient of the displacement fluctuation modes, see Eq. 23.
} 
Having definitions (41) and (42) at hand, the approximation of Eq.(22) by Gauss quadrature can be written as:

$$
\begin{aligned}
& \int_{\Omega} \boldsymbol{B}_{i}^{* T}(\mathbf{x}) \boldsymbol{\sigma}(\mathbf{x} ; \cdot) d \Omega \approx \sum_{g=1}^{n_{g}} w_{g} \boldsymbol{B}_{i}^{* T}\left(\mathbf{x}_{g}\right) \boldsymbol{\sigma}\left(\mathbf{x}_{g} ; \cdot\right)=0 \\
& \Rightarrow \mathcal{B}_{i}^{* T} \boldsymbol{W} \mathcal{S}=0, \quad i=1,2 \ldots n_{u}
\end{aligned}
$$

where $\boldsymbol{W}$ is a diagonal matrix containing the weights at each Gauss point:

$$
\boldsymbol{W}:=\left[\begin{array}{ccccc}
w_{1} \boldsymbol{I} & \mathbf{0} & \mathbf{0} & \cdots & \mathbf{0} \\
\mathbf{0} & w_{2} \boldsymbol{I} & \mathbf{0} & \cdots & \mathbf{0} \\
\vdots & \vdots & \vdots & \vdots & \vdots \\
\mathbf{0} & \mathbf{0} & \mathbf{0} & \mathbf{0} & w_{n_{g}} \boldsymbol{I}
\end{array}\right]
$$

(here, $\boldsymbol{I}$ denotes the $s \times s$ identity matrix). Assuming that $w_{g}>0(g=$ $\left.1,2 \ldots n_{g}\right)$ - Gauss quadrature rules with negative weights are excluded from our considerations - , and using the Cholesky decomposition of $\boldsymbol{W}(\boldsymbol{W}=$ $\boldsymbol{W}^{1 / 2} \boldsymbol{W}^{1 / 2}$ ), one can reexpress Eq.(43) as

$$
\mathcal{B}^{* T} \boldsymbol{W} \mathcal{S}=\left(\mathcal{B}^{* T} \boldsymbol{W}^{1 / 2}\right)\left(\boldsymbol{W}^{1 / 2} \mathcal{S}\right)=\mathbf{0} .
$$

Defining now the weighted global stress vector and weighted matrix of strain modes as

$$
\boldsymbol{\Sigma}:=\boldsymbol{W}^{1 / 2} \mathcal{S}=\left[\begin{array}{llll}
\sqrt{w_{1}} \boldsymbol{\sigma}^{T}\left(\mathbf{x}_{1} ; \cdot\right) & \sqrt{w_{2}} \boldsymbol{\sigma}^{T}\left(\mathbf{x}_{2} ; \cdot\right) & \cdots & \sqrt{w_{n_{g}}} \boldsymbol{\sigma}^{T}\left(\mathbf{x}_{n_{g}} ; \cdot\right)
\end{array}\right]^{T}
$$

and

$$
\mathbb{B}^{*}:=\boldsymbol{W}^{1 / 2} \mathcal{B}^{*}=\left[\begin{array}{llll}
\sqrt{w_{1}} \boldsymbol{B}^{* T}\left(\mathbf{x}_{1}\right) & \sqrt{w_{2}} \boldsymbol{B}^{* T}\left(\mathbf{x}_{2}\right) & \cdots & \sqrt{w_{n_{g}}} \boldsymbol{B}^{* T}\left(\mathbf{x}_{n_{g}}\right)
\end{array}\right]^{T}
$$

respectively, and inserting these definitions into Eq.(41), one finally arrives at:

$$
\mathbb{B}^{* T} \boldsymbol{\Sigma}=\mathbf{0}
$$

or equivalently,

$$
\mathbb{B}_{i}^{* T} \boldsymbol{\Sigma}=0, \quad i=1,2 \ldots n_{u},
$$

which shows that any statically admissible weighted stress vector is orthogonal, in the sense of the standard euclidean inner product, to the weighted strain modes $\mathbb{B}_{i}^{* T}\left(i=1,2 \ldots n_{u}\right)$. 
Comparing Eq.(48) with Eq.(32), it becomes clear that $\mathbb{B}^{* T}$ plays the same role as operator $\boldsymbol{G}$ in Eq.(32). In analogy with Eq.(33), thus, we can write

$$
\mathbb{R}^{n_{g} \cdot s}=\mathcal{N}\left(\mathbb{B}^{* T}\right) \oplus \operatorname{Range}\left(\mathbb{B}^{*}\right)
$$

where $\mathcal{N}\left(\mathbb{B}^{* T}\right)$ and Range $\left(\mathbb{B}^{*}\right)$ denote the null space and the range (or column space) of $\mathbb{B}^{* T}$ and $\mathbb{B}^{*}$, respectively, and consequently decompose any $\boldsymbol{\Sigma} \in$ $\mathbb{R}^{n_{g} \cdot s}$ as

$$
\Sigma=\Sigma^{a d}+\Sigma^{i n}
$$

with $\Sigma^{\boldsymbol{a d}} \in \mathcal{N}\left(\mathbb{B}^{* T}\right)$ and $\boldsymbol{\Sigma}^{\boldsymbol{i n}} \in \operatorname{Range}\left(\mathbb{B}^{*}\right)$. As in the continuous case (see Eq.(35)), the statically admissible component $\boldsymbol{\Sigma}^{\boldsymbol{a d}}$ is now approximated by a linear combination of POD basis vectors obtained from converged stress snapshots $^{12}$ :

$$
\Sigma^{a d} \approx \Sigma^{*}=\sum_{i=1}^{n_{\sigma}} c_{i}^{a d} \Psi_{i}=\Psi c^{a d}
$$

where $\boldsymbol{\Psi}=\left[\begin{array}{llll}\boldsymbol{\Psi}_{1} & \boldsymbol{\Psi}_{2} & \cdots & \boldsymbol{\Psi}_{n_{\sigma}}\end{array}\right]$ denotes the (weighted) stress basis matrix and $\boldsymbol{c}^{a d} \in \mathbb{R}^{n_{\sigma}}$ stands for the vector of modal coefficients associated to such a basis matrix. Likewise, since the non-equilibrated component $\boldsymbol{\Sigma}^{\boldsymbol{i n}}$ pertains to the column space of $\mathbb{B}^{*}$, we can directly write

$$
\Sigma^{i n}=\mathbb{B}^{*} c^{i n}
$$

where $\boldsymbol{c}^{i n} \in \mathbb{R}^{n_{u}}$. The low-dimensional (weighted) stress vector $\boldsymbol{\Sigma}^{\text {ex* }}$ required in the proposed integration method is finally obtained as the sum of Eq.(53) and Eq.(52).

$$
\Sigma \approx \Sigma^{e x *}=\Psi c^{a d}+\mathbb{B}^{*} c^{i n}
$$

or in a more compact format:

$$
\Sigma^{e x *}=\Psi^{e x} \boldsymbol{c}
$$

where

$$
\Psi^{e x}:=\left[\begin{array}{ll}
\Psi & \mathbb{B}^{*}
\end{array}\right]
$$

\footnotetext{
${ }^{12}$ The methodology for obtaining these modes using the SVD is similar to that explained in Section 3.2 for the displacement fluctuation modes.
} 
and

$$
\boldsymbol{c}=\left[\begin{array}{l}
\boldsymbol{c}^{a d} \\
\boldsymbol{c}^{i n}
\end{array}\right]
$$

The matrix $\Psi^{e x} \in \mathbb{R}^{n_{g} \cdot s \times\left(n_{u}+n_{\sigma}\right)}$ defined by Eq.(56) will be hereafter called the expanded basis matrix for the (weighted) stresses, whereas $c \in \mathbb{R}^{n_{\sigma}+n_{u}}$ will be correspondingly termed the expanded vector of modal coefficients. Inserting approximation (54) into Eq.(48), and considering that $\mathbb{B}^{* T} \boldsymbol{\Psi}=\mathbf{0}$ and that $\mathbb{B}^{* T}$ is a full rank matrix, one finally arrives at the same equilibrium condition derived in the continuum case (see Eq. 39):

$$
c^{i n}\left(U^{*}, \epsilon_{M}\right)=\mathbf{0}
$$

Once the above equation is solved for $\boldsymbol{U}^{*}$, the desired equilibrated stress vector $\boldsymbol{\Sigma}^{*}$ is obtained by evaluating Eq.(52):

$$
\Sigma^{*}=\Psi c^{a d}\left(\boldsymbol{U}^{*}, \boldsymbol{\epsilon}_{M}\right)
$$

\section{The High-Performance ROM}

The next step in the development of the proposed integration scheme is to deduce closed-form expressions for the vectors of modal coefficients $\boldsymbol{c}^{a d} \in \mathbb{R}^{n_{\sigma}}$ and $\boldsymbol{c}^{i n} \in \mathbb{R}^{n_{u}}$ in terms of the stress values computed at a set of $p=\mathcal{O}\left(n_{u}\right)$ pre-specified sampling points (to be chosen among the set of Gauss points of the underlying finite element mesh). To this end, we need first to introduce some notation and terminology.

\subsection{Gappy vectors}

Let $\mathcal{I}=\left\{\mathcal{I}_{1}, \mathcal{I}_{2} \ldots \mathcal{I}_{p}\right\} \subset\left\{1,2 \cdots n_{g}\right\}$ denote the set of indices of sampling points. Notationally, we write $\hat{\boldsymbol{\Sigma}}_{(\mathcal{I})} \in \mathbb{R}^{p \cdot s}$ to designate the subvector of $\boldsymbol{\Sigma}$ containing the rows associated to these sampling points; viz.:

$$
\hat{\boldsymbol{\Sigma}}_{(\mathcal{I})}:=\left[\begin{array}{llll}
\sqrt{w_{\mathcal{I}_{1}}} \boldsymbol{\sigma}^{T}\left(\mathbf{x}_{\mathcal{I}_{1}}, \cdot\right) & \sqrt{w_{\mathcal{I}_{2}}} \boldsymbol{\sigma}^{T}\left(\mathbf{x}_{\mathcal{I}_{2}}, \cdot\right) & \cdots & \sqrt{w_{\mathcal{I}_{p}}} \boldsymbol{\sigma}^{T}\left(\mathbf{x}_{\mathcal{I}_{p}}, \cdot\right)
\end{array}\right]^{T}
$$

(When confusion is not apt to arise, the parenthetical subscript indicating the set of sampling indices will be dropped, and we shall simply write $\hat{\Sigma}$ ). It proves conceptually advantageous to regard this restricted or "gappy" a terminology that goes back to the work of Everson et al. [28] - stress vector $\hat{\Sigma}_{(\mathcal{I})}$ as the result of the application of a certain boolean operator 
$\mathcal{P}_{(\mathcal{I})}: \mathbb{R}^{n_{g} \cdot s} \rightarrow \mathbb{R}^{p \cdot s}$ over the full vector $\boldsymbol{\Sigma}$ (i.e., $\left.\hat{\boldsymbol{\Sigma}}=\mathcal{P}_{(\mathcal{I})} \boldsymbol{\Sigma}\right)$. We call $\mathcal{P}_{(\mathcal{I})}$ the selection operator associated to sampling indices $\mathcal{I}$. This operator can be of course applied to any $\mathbf{Y} \in \mathbb{R}^{\mathbf{n}_{\mathbf{g}} \cdot \mathbf{s} \times \mathbf{z}}(z \in \mathbb{N})$. For instance, the restricted matrix of weighted strain modes is defined as $\widehat{\mathbb{B}}^{*}:=\mathcal{P}_{(\mathcal{I})} \mathbb{B}^{*}$. Furthermore, it is straighforward to show that

$$
\mathcal{P}_{(\mathcal{I})} \mathcal{P}_{(\mathcal{I})}^{T}=\boldsymbol{I}
$$

(here $\boldsymbol{I}$ is the $\left(n_{g} \cdot s\right) \mathrm{x}\left(n_{g} \cdot s\right)$ identity matrix) and that

$$
\mathcal{P}_{(\mathcal{I})}(\mathrm{AY})=\left(\mathcal{P}_{(\mathcal{I})} \mathbf{A} \mathcal{P}_{(\mathcal{I})}^{\mathrm{T}}\right)\left(\mathcal{P}_{(\mathcal{I})} \mathbf{Y}\right)
$$

for any $\mathbf{A} \in \mathbb{R}^{\mathbf{n}_{\mathbf{g}} \cdot \mathbf{s} \times \mathbf{n}_{\mathbf{g}} \cdot \mathbf{s}}$ and $\mathbf{Y} \in \mathbb{R}^{\mathbf{n}_{\mathbf{g}} \cdot \mathbf{s} \times \mathbf{z}}$.

\subsection{Least-squares fit}

In the spirit of classical polynomial quadrature, such as Newton-Cotes formulae [36], the modal coefficients $\boldsymbol{c}^{a d} \in \mathbb{R}^{n_{\sigma}}$ and $\boldsymbol{c}^{i n} \in \mathbb{R}^{n_{u}}$ are determined by fitting the low-dimensional approximation (54) to the weighted stresses calculated at the pre-specified sampling points. It should be noticed that, the variable subject to approximation - the stress - being a vector-valued function, the total number of discrete points to be fitted does not coincide with the number of spatial sampling points $(p)$, but rather is equal to the product of such a number times the number of stress components $(s)$. The well-posedness of the fitting problem, thus, demands that $p \cdot s \geq n_{\sigma}+n_{u}$, i.e., the number of discrete points must be equal or greater than the number of parameters to be adjusted. For the equality to hold, both $n_{\sigma}+n_{u}$ and $p$ have to be multiple of $s$; thus, an exact fit is in general not possible for arbitrary values of $n_{\sigma}$ and $n_{u}$, and recourse to an approximate fit is to be made. In this respect, we follow here the standard approach of using a least-squares, best-fit criterion, i.e., minimization of the squares of the deviations between "observed" $(\hat{\boldsymbol{\Sigma}})$ and fitted $\left(\hat{\boldsymbol{\Sigma}}^{e x *}=\hat{\boldsymbol{\Psi}} \boldsymbol{a}+\hat{\mathbb{B}}^{*} \boldsymbol{b}\right)$ values (in our context, "observed" signifies "calculated through the pertinent constitutive equation"). This minimization problem can be stated as:

$$
\boldsymbol{c}=\left[\begin{array}{c}
\boldsymbol{c}^{a d} \\
\boldsymbol{c}^{i n}
\end{array}\right]=\arg \min _{\boldsymbol{a} \in \mathbb{R}^{n_{\sigma}, \boldsymbol{b} \in \mathbb{R}^{n_{u}}}}\left\|\hat{\boldsymbol{\Sigma}}-\left(\hat{\mathbf{\Psi}} \boldsymbol{a}+\hat{\mathbb{B}}^{*} \boldsymbol{b}\right)\right\|
$$

where $\|\cdot\|$ stands for the standard euclidean norm. Let $\hat{\Psi}^{e x}=\mathcal{P}_{(\mathcal{I})} \Psi^{e x}=$ $\left[\begin{array}{ll}\hat{\Psi} & \mathbb{B}^{*}\end{array}\right]$ be the gappy expanded basis matrix, and suppose that the sampling indices $\mathcal{I}$ have been chosen so that $\hat{\mathbf{\Psi}}^{e \boldsymbol{x}}$ has full rank, i.e.:

$$
\operatorname{rank}\left(\hat{\mathbf{\Psi}}^{\text {ex }}\right)=\operatorname{rank}\left(\left[\begin{array}{ll}
\mathbf{\Psi} & \hat{\mathbb{B}}^{*}
\end{array}\right]\right)=n_{\sigma}+n_{u} .
$$


Then, it can be shown (see, for instance, Ref. [23]) that the solution of this standard, least-squares problem is provided by the following vector of coefficients:

$$
c=\left[\begin{array}{l}
c^{a d} \\
c^{i n}
\end{array}\right]=\hat{\Psi}^{e x \dagger} \hat{\Sigma}
$$

where

$$
\hat{\boldsymbol{\Psi}}^{e x \dagger}:=\overbrace{\left(\hat{\boldsymbol{\Psi}}^{\boldsymbol{e x}^{T}} \hat{\boldsymbol{\Psi}}^{\boldsymbol{e x}}\right)^{-1}}^{\hat{\mathbf{M}}^{-1}} \hat{\boldsymbol{\Psi}}^{\boldsymbol{e x}^{T}}
$$

is the so-called pseudo-inverse of matrix $\hat{\Psi}^{\boldsymbol{e x}}$.

Recall that our ultimate aim is to derive closed-form expressions for $\boldsymbol{c}^{\text {in }}$ and $\boldsymbol{c}^{a d}$ as functions of $\hat{\boldsymbol{\Sigma}}$. Thus, it remains to extricate these two subvectors from expression (65). This can be done by first partitioning both $\hat{\boldsymbol{M}}=\hat{\boldsymbol{\Psi}}^{\boldsymbol{e x}} \hat{\boldsymbol{\Psi}}^{T} \hat{\boldsymbol{\Psi}}^{\boldsymbol{x}}$ and $\hat{\boldsymbol{\Psi}}^{\boldsymbol{e \boldsymbol { x } ^ { T }}}$ in terms of the gappy stress basis matrix $\hat{\boldsymbol{\Psi}}$ and the gappy matrix of strain modes $\hat{\mathbb{B}}^{*}$ :

$$
\boldsymbol{c}=\left[\begin{array}{c}
\boldsymbol{c}^{a d} \\
\boldsymbol{c}^{i n}
\end{array}\right]=\left[\begin{array}{cc}
\hat{\boldsymbol{\Psi}}^{T} \hat{\mathbf{\Psi}} & \hat{\mathbf{\Psi}}^{T} \hat{\mathbb{B}}^{*} \\
\hat{\mathbb{B}}^{*^{T}} \hat{\boldsymbol{\Psi}} & \hat{\mathbb{B}}^{*^{T}} \hat{\mathbb{B}}^{*}
\end{array}\right]^{-1}\left[\begin{array}{c}
\hat{\mathbf{\Psi}}^{T} \\
\hat{\mathbb{B}}^{*^{T}}
\end{array}\right] \hat{\boldsymbol{\Sigma}} .
$$

Invoking the blockwise inverse formula for $2 \mathrm{x} 2$ block symmetric matrices [11], and upon tedious algebra - that has been relegated to Appendix $\mathrm{C}$ - one finally arrives at the following expressions for $\boldsymbol{c}^{a d}$ and $\boldsymbol{c}^{i n}$

$$
\begin{gathered}
\boldsymbol{c}^{a d}=\hat{\boldsymbol{\Psi}}^{\dagger}\left(\hat{\Sigma}-\hat{\mathbb{B}}^{*} \boldsymbol{c}^{i n}\right) \\
\boldsymbol{c}^{i n}=\mathbf{S}^{-\mathbf{1}} \hat{\mathbb{B}}^{*^{\mathbf{T}}}\left(\boldsymbol{I}-\hat{\boldsymbol{\Psi}} \hat{\Psi}^{\dagger}\right) \hat{\boldsymbol{\Sigma}}
\end{gathered}
$$

where $\hat{\Psi}^{\dagger}$ denotes the pseudoinverse of the gappy stress basis matrix $\hat{\Psi}$ :

$$
\hat{\mathbf{\Psi}}^{\dagger}=\left(\hat{\mathbf{\Psi}}^{T} \hat{\boldsymbol{\Psi}}\right)^{-1} \hat{\boldsymbol{\Psi}}^{T}
$$

and $\mathbf{S}:=\hat{\mathbb{B}}^{* \mathbf{T}}\left(\boldsymbol{I}-\hat{\boldsymbol{\Psi}} \hat{\boldsymbol{\Psi}}^{\dagger}\right.$ ) (note that $\mathbf{S}$ is invertible by virtue of the hypothesis represented by Eq.(64)).

\subsubsection{Reconstruction matrix}

Let us first examine expression (68) for the modal coefficients $\boldsymbol{c}^{a d}$ - those that multiply the statically admissible component of the global stress vector. 
Since, at the solution, $\boldsymbol{c}^{i n}=\mathbf{0}$, we have that:

$$
c^{a d}=\hat{\Psi}^{\dagger}(\hat{\Sigma}-\hat{\mathbb{B}}^{*} \overbrace{c^{i n}}^{=0})=\hat{\Psi}^{\dagger} \hat{\Sigma} .
$$

(Notice that this result can also be obtained by directly solving minimization problem (63) with $\boldsymbol{b}=\mathbf{0}$ ). Substitution of this equation into Eq.(59) yields:

$$
\Sigma^{*}=\Psi c^{a d}=\overbrace{\Psi \hat{\Psi}^{\dagger}}^{R} \hat{\Sigma}=R \hat{\Sigma},
$$

where

$$
\boldsymbol{R}:=\boldsymbol{\Psi} \hat{\Psi}^{\dagger}=\boldsymbol{\Psi}\left(\hat{\boldsymbol{\Psi}}^{T} \hat{\boldsymbol{\Psi}}\right)^{-1} \hat{\Psi}^{T} .
$$

Inspection of Eq.(72) reveals that the matrix $\boldsymbol{R} \in \mathbb{R}^{n_{g} \cdot s \times p \cdot s}$ defined above is the operator that allows one to reconstruct the (weighted) statically admissible stress vector $\boldsymbol{\Sigma}^{*} \in \mathbb{R}^{n_{g} \cdot s}$ using only the (weighted) stress values $\left(\hat{\Sigma} \in \mathbb{R}^{p \cdot s}\right)$ calculated at the pre-selected sampling points $\mathcal{I}$. For this reason, we shall use the term weighted reconstruction matrix (or simply reconstruction matrix) to refer to this operator. It must be emphasized here that this matrix only depends on the POD stress basis matrix $\boldsymbol{\Psi}$ and on the selected sampling indices $\mathcal{I}$-i.e., it is independent of the input parameter, the macro-strain $\boldsymbol{\epsilon}_{\boldsymbol{M}}$-and, therefore, it can be pre-computed offline.

\section{3. "Hyperreduced" RVE equilibrium equation}

As for the expression for the set of "statically inadmissible" coefficients $\boldsymbol{c}^{i n} \in \mathbb{R}^{n_{u}}$, we know that, at the solution, these coefficients must vanish; thus, from Eq.(69), we have

$$
c^{i n}\left(\boldsymbol{U}^{*}, \boldsymbol{\epsilon}_{\boldsymbol{M}}\right)=\mathbf{S}^{-\mathbf{1}} \hat{\mathbb{B}}^{*^{\mathbf{T}}}\left(\boldsymbol{I}-\hat{\boldsymbol{\Psi}} \hat{\Psi}^{\dagger}\right) \hat{\Sigma}\left(\boldsymbol{U}^{*}, \boldsymbol{\epsilon}_{\boldsymbol{M}}\right)=\mathbf{0} .
$$

Since $\mathbf{S}$ is a nonsingular matrix, the above condition is equivalent to

$$
\hat{\mathbb{B}}^{*^{T}}\left(\boldsymbol{I}-\hat{\boldsymbol{\Psi}} \hat{\Psi}^{\dagger}\right) \hat{\Sigma}\left(\boldsymbol{U}^{*}, \boldsymbol{\epsilon}_{\boldsymbol{M}}\right)=\mathbf{0} .
$$

Furthermore, examination of Eq.(73) and Eq.(75) readily shows that the bracketed term $\hat{\Psi} \hat{\Psi}^{\dagger}$ in Eq.(75) is nothing but the submatrix of the reconstruction matrix $\boldsymbol{R}$ formed by the rows associated to sampling points $\mathcal{I}$, i.e.:

$$
\hat{\Psi} \hat{\Psi}^{\dagger}=\mathcal{P}_{(\mathcal{I})}\left(\Psi \hat{\Psi}^{\dagger}\right)=\mathcal{P}_{(\mathcal{I})} R=\hat{R}
$$


Substitution of expression (76) into Eq.(75) finally leads to:

$$
\hat{\mathbb{B}}^{*^{T}}(\boldsymbol{I}-\hat{\boldsymbol{R}}) \hat{\boldsymbol{\Sigma}}\left(\boldsymbol{U}^{*}, \boldsymbol{\epsilon}_{\boldsymbol{M}}\right)=\mathbf{0} .
$$

As previously noted (see Figure 2), the purpose of enforcing condition $\boldsymbol{c}^{i n}\left(\boldsymbol{U}^{*}, \boldsymbol{\epsilon}_{\boldsymbol{M}}\right)=\mathbf{0}$ is to ensure that the stress solution lies entirely in the space of equilibrated stresses. Equation (77) can be viewed, thus, as the "hyperreduced" form of the original RVE equilibrium equation.

Observation 6.1. The "hyperreduced" qualifier - coined by D. Ryckelynck [58, 59] - is used here to indicate that Eq.(77) is the result of two subsequent steps of complexity reduction: firstly, in the number of degrees of freedom (when passing from the finite element model to the ROM that employs standard Gauss quadrature), and, secondly, in the number of integration points (when passing from this standard ROM to what we have baptized" "HighPerformance" ROM ). This double complexity reduction can be better appreciated by rephrasing both Eq.(77) and the FE equation (9) in a format similar to that of Eq.(48), viz.:

$$
\overbrace{\hat{\mathbb{B}}^{*^{T}}(\boldsymbol{I}-\hat{\boldsymbol{R}})}^{\hat{\mathbb{B}}^{* *^{T}}} \hat{\boldsymbol{\Sigma}}=\hat{\mathbb{B}}^{*^{*}} \hat{\boldsymbol{\Sigma}}=\mathbf{0},
$$

and

$$
\sum_{g=1}^{n_{g}} w_{g} \boldsymbol{B}^{T}\left(\boldsymbol{x}_{g}\right) \boldsymbol{\sigma}\left(\boldsymbol{x}_{g}, ;\right)=\mathbb{B}^{T} \boldsymbol{\Sigma}=\mathbf{0},
$$

respectively (here, $\mathbb{B} \in \mathbb{R}^{n_{g} \cdot s \times n \cdot d}$ is the finite element counterpart of $\mathbb{B}^{*}$, defined in Eq.(42)). With Eq.(79), Eq.(48) and Eq.(78) at our disposal, the abovementioned process of complexity reduction can be symbolically represented as

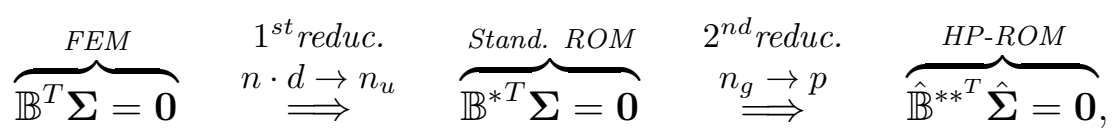

\footnotetext{
13 The term High-Performance, Reduced-Order Model (HP-ROM) is used to highlight the tremendous gains in performance that affords this model over the standard ROM, let alone over the full-order, finite model. In the numerical example shown in Section 9, we report speedup factors of above three order of magnitudes.
} 
the relation between $\mathbb{B} \in \mathbb{R}^{n_{g} \cdot s \times n \cdot d}, \mathbb{B}^{*} \in \mathbb{R}^{n_{g} \cdot s \times n_{u}}$ and $\hat{\mathbb{B}}^{* *} \in \mathbb{R}^{p \cdot s \times n_{u}}$ being $\mathbb{B}^{*}=\mathbb{B} \Phi$ and

$$
\hat{\mathbb{B}}^{* *}:=(\boldsymbol{I}-\hat{\boldsymbol{R}}) \hat{\mathbb{B}}^{*}=(\boldsymbol{I}-\hat{\boldsymbol{R}}) \mathcal{P}_{(\mathcal{I})} \mathbb{B}^{*},
$$

with $p=\mathcal{O}\left(n_{u}\right)<<n_{g}=\mathcal{O}(n)$. It is interesting to see how the reduction in complexity of the RVE equilibrium equation is reflected in the gradual reduction of the dimensions of the " $B$ " operators that act on the weighted vector of stresses.

\subsubsection{Physical interpretation}

Aside from a "compressed" version of the original, full-order cell condition, the hyperreduced equation (77) can be alternatively interpreted as a balance between "observed" and "fitted" internal forces at the selected sampling points. Such an interpretation becomes readily identifiable by realizing that the product $\hat{\boldsymbol{R}} \hat{\boldsymbol{\Sigma}}$ appearing in Eq.(77) is but the (weighted) vector of fitted stresses at the selected sampling points. Indeed, by virtue of Eq.(72) and, considering the properties of the selection operator $\mathcal{P}_{(\mathcal{I})}$, we have that

$$
\hat{\boldsymbol{R}} \hat{\Sigma}=\mathcal{P}_{(\mathcal{I})}(\boldsymbol{R} \hat{\Sigma})=\mathcal{P}_{(\mathcal{I})} \boldsymbol{\Sigma}^{*}=\hat{\Sigma}^{*}
$$

Using the above equality, Eq.(77) is expressible as $\hat{\mathbb{B}}^{*^{T}} \hat{\boldsymbol{\Sigma}}=\hat{\mathbb{B}}^{*^{T}} \hat{\boldsymbol{\Sigma}}^{*}$, or, reverting to the original, summation notation as

$$
\sum_{j \in \mathcal{I}} w_{j} \boldsymbol{B}^{* T}\left(\mathbf{x}_{j}\right) \boldsymbol{\sigma}\left(\mathbf{x}_{j} ; \cdot\right)=\sum_{j \in \mathcal{I}} w_{j} \boldsymbol{B}^{* T}\left(\mathbf{x}_{j}\right) \boldsymbol{\sigma}^{*}\left(\mathbf{x}_{j} ; \cdot\right) .
$$

Note that both sides of the above equation represent the same physical quantity, namely, the sum of internal forces, in reduced coordinates, at the sampling Gauss points $\left\{\mathbf{x}_{\mathcal{I}_{1}}, \mathbf{x}_{\mathcal{I}_{2}} \cdots \mathbf{x}_{\mathcal{I}_{p}}\right\}$. The difference lies in the stresses employed for computing these internal forces. In the left-hand side, they are calculated using "observed" stresses $\boldsymbol{\sigma}$ - stresses that arises directly from evaluating the corresponding constitutive equation-, whereas, in the righthand side, "fitted" stresses $\boldsymbol{\sigma}^{*}$ are used - that is, stresses obtained from fitting the approximation constructed using the POD stress basis functions $\boldsymbol{\Psi}_{1}, \boldsymbol{\Psi}_{2} \ldots \boldsymbol{\Psi}_{n_{\sigma}}$ to the observed data. Thus, the HP-ROM equilibrium condition (83) is telling us that, at the solution, the sum of internal forces - at the pre-selected sampling points - computed using either observed or fitted stresses ${ }^{14}$ must coincide.

\footnotetext{
${ }^{14}$ It should be mentioned in this respect that, in general, $\boldsymbol{\sigma}^{*}\left(\mathbf{x}_{j} ; \cdot\right) \neq \boldsymbol{\sigma}\left(\mathbf{x}_{j} ; \cdot\right)$ since the
} 


\subsection{Jacobian matrix}

Needless to say, the dependence of the stresses on the reduced vector of reduced displacement fluctuations $\boldsymbol{U}^{*}$ is in general non-linear, and, thereby, an iterative method is required for solving Eq.(77). Here we employ the standard Newton-Raphson procedure. The iterative scheme corresponding to this procedure is given by the following expression (the parenthetical superscript indicates iteration number):

$$
\boldsymbol{U}^{*(k+1)}=\boldsymbol{U}^{*(k)}-\boldsymbol{K}^{*(k)^{-1}} \boldsymbol{F}^{*(k)},
$$

where

$$
\boldsymbol{F}^{*(k)}=\hat{\mathbb{B}}^{*^{T}}(\boldsymbol{I}-\hat{\boldsymbol{R}}) \hat{\boldsymbol{\Sigma}}\left(\boldsymbol{\epsilon}_{\boldsymbol{M}}, \boldsymbol{U}^{*(k)}\right)
$$

and

$$
\boldsymbol{K}^{*(k)}=\hat{\mathbb{B}}^{*^{T}}(\boldsymbol{I}-\hat{\boldsymbol{R}}) \hat{\mathbb{C}}\left(\boldsymbol{\epsilon}_{\boldsymbol{M}}, \boldsymbol{U}^{*(k)}\right) \hat{\mathbb{B}}^{*} .
$$

In the above equation, $\hat{\mathbb{C}} \in \mathbb{R}^{p \cdot s \times p \cdot s}$ denotes a block diagonal matrix containing the algorithmic, constitutive tangent matrices at each sampling point:

$$
\hat{\mathbb{C}}:=\left[\begin{array}{ccccc}
C\left(\mathrm{x}_{\mathcal{I}_{1}} ; \cdot\right) & \mathbf{0} & \mathbf{0} & \cdots & \mathbf{0} \\
\mathbf{0} & C\left(\mathrm{x}_{\mathcal{I}_{2}} ; \cdot\right) & \mathbf{0} & \cdots & \mathbf{0} \\
\vdots & \vdots & \vdots & \vdots & \vdots \\
\mathbf{0} & \mathbf{0} & \mathbf{0} & \mathbf{0} & \boldsymbol{C}\left(\mathrm{x}_{\mathcal{I}_{p}} ; \cdot\right)
\end{array}\right] .
$$

\subsubsection{Positive definiteness}

Because of its relevance in the overall robustness of the proposed method, it is worthwhile at this point to digress and discuss thoroughly the spectral properties of the Jacobian matrix represented by Eq.(86). In particular, it would be interesting to ascertain whether positive definiteness of the algorithmic tangent matrices $\boldsymbol{C}\left(\mathrm{x}_{\mathcal{I}_{1}} ; \cdot\right), \boldsymbol{C}\left(\mathbf{x}_{\mathcal{I}_{2}} ; \cdot\right), \cdots \boldsymbol{C}\left(\mathbf{x}_{\mathcal{I}_{p}}\right)$ at the selected sampling points, and thus of matrix $\hat{\mathbb{C}}$, ensures positive definiteness of the Jacobian matrix $\boldsymbol{K}^{*}$-as it occurs when using classical Gauss quadrature rules with positive weights-, and, if not, which remedies can be applied to obtain such desirable property.

number of data items to be fitted $(p \cdot s)$ is always greater than the number of stress modes $\left(n_{\sigma}\right)$. Observed and fitted stresses coincide only when the stress vector $\boldsymbol{\Sigma}$ one wishes to approximate pertains to the column space of the stress basis matrix $\Psi$ 
Positive definiteness of the Jacobian matrix (86) requires that the function defined as

$$
F(\mathbf{U})=\mathbf{U}^{\mathbf{T}} \boldsymbol{K}^{*} \mathbf{U}=\left(\hat{\mathbb{B}}^{*} \mathbf{U}\right)^{\mathbf{T}}(\boldsymbol{I}-\hat{\boldsymbol{R}}) \hat{\mathbb{C}}\left(\hat{\mathbb{B}}^{*} \mathbf{U}\right)
$$

be positive for all non-zero $\mathbf{U} \in \mathbb{R}^{\mathbf{n}_{\mathbf{u}}}$. Since $\hat{\mathbb{B}}^{*}$ is a full rank matrix - by virtue of Eq.(64) - , condition $F(\mathbf{U})>\mathbf{0}$ is equivalent to:

$$
G(\mathbf{V})=\mathbf{V}^{\mathbf{T}}(\boldsymbol{I}-\hat{\boldsymbol{R}}) \hat{\mathbb{C}} \mathbf{V}>\mathbf{0}
$$

for all non-zero $\mathbf{V} \in \operatorname{Range}\left(\hat{\mathbb{B}}^{*}\right)$.

To go further, we need to demonstrate that $\hat{\boldsymbol{R}} \in \mathbb{R}^{n_{g} \cdot s \times n_{g} \cdot s}$ - recall that $\hat{\boldsymbol{R}}$ is the matrix that maps the vector of "observed" stresses $\hat{\boldsymbol{\Sigma}}$ to the vector of fitted stresses $\hat{\mathbf{\Sigma}}^{*}$ - actually represents an orthogonal projection ${ }^{15}$ onto the column space of the gappy stress basis matrix $\hat{\Psi}$. This can be shown by simply noting that $\hat{\boldsymbol{R}}$ is, on the one hand, symmetric:

$$
\hat{\boldsymbol{R}}^{T}=\left(\hat{\boldsymbol{\Psi}}\left(\hat{\boldsymbol{\Psi}}^{T} \hat{\boldsymbol{\Psi}}\right)^{-1} \hat{\boldsymbol{\Psi}}^{T}\right)^{T}=\hat{\boldsymbol{\Psi}}\left(\hat{\boldsymbol{\Psi}}^{T} \hat{\boldsymbol{\Psi}}\right)^{-T} \hat{\boldsymbol{\Psi}}^{T}=\hat{\boldsymbol{R}}
$$

and, on the other hand, idempotent:

$$
\hat{\boldsymbol{R}}^{2}=\left(\hat{\mathbf{\Psi}} \hat{\boldsymbol{\Psi}}^{\dagger}\right)^{2}=\hat{\mathbf{\Psi}} \overbrace{\left(\hat{\mathbf{\Psi}}^{T} \hat{\boldsymbol{\Psi}}\right)^{-1} \hat{\boldsymbol{\Psi}}^{T} \hat{\mathbf{\Psi}}}^{=\boldsymbol{I}}\left(\hat{\mathbf{\Psi}}^{T} \hat{\boldsymbol{\Psi}}\right)^{-1} \hat{\boldsymbol{\Psi}}^{T}=\hat{\mathbf{\Psi}}\left(\hat{\boldsymbol{\Psi}}^{T} \hat{\boldsymbol{\Psi}}\right)^{-1} \hat{\boldsymbol{\Psi}}^{T}=\hat{\boldsymbol{R}} .
$$

With this property at hand, we can decompose any $\mathbf{V} \in \operatorname{Range}\left(\hat{\mathbb{B}}^{*}\right)$ as

$$
\mathbf{V}=\mathbf{V}^{\|}+\mathbf{V}^{\perp}
$$

where $\mathbf{V}^{\|}=\hat{\boldsymbol{R}} \mathbf{V} \in \operatorname{Range}(\hat{\boldsymbol{\Psi}})$ - the component of $\mathbf{V}$ along the column space of $\hat{\mathbf{\Psi}}$ - and $\mathbf{V}^{\perp}=(\boldsymbol{I}-\hat{\boldsymbol{R}}) \mathbf{V}$ - the component of $\mathbf{V}$ along the orthogonal complement of Range $(\hat{\Psi})$. Introducing the above decomposition into Eq.(89), we arrive at

$$
G=\mathbf{V}^{\perp T} \hat{\mathbb{C}} \mathbf{V}^{\perp}+\mathbf{V}^{\perp} \hat{\mathbb{C}}^{\|}
$$

While the first term $\mathbf{V}^{\perp^{T}} \hat{\mathbb{C}} \mathbf{V}^{\perp}$ in the preceding equation is, in virtue of the positive definiteness of $\hat{\mathbb{C}}$, eminently positive for all nonzero $\mathbf{V}^{\perp} \in \mathbb{R}^{\mathbf{p} \cdot \mathbf{s}}$, nothing can be said in principle about the second term $\mathbf{V}^{\perp^{T}} \hat{\mathbb{C}} \mathbf{V}^{\|}$: numerical experience shows that the sign and relative magnitude of this term depends further on the chosen set of sampling indices $\mathcal{I}$.

\footnotetext{
${ }^{15} \hat{\boldsymbol{R}}$ is the so-called "hat" matrix of linear regression models [52].
} 
Remark 6.1. From the above observation, it follows that the positive definiteness of the Jacobian matrix $\boldsymbol{K}^{*}$ is determined, not only by the spectral properties of $\hat{\mathbb{C}}$, but - not surprisingly - also by the number and the location within the RVE of the sampling points employed in the integration.

The foregoing remark naturally leads to wonder whether it is possible to select the sampling indices $\mathcal{I}$ so as to ensure the positive definiteness of $\boldsymbol{K}^{*}$ (assuming, obviously, that $\hat{\mathbb{C}}$ enjoys this property). To shed light on this question, let us first divide Eq.(93) by $\mathbf{V}^{\perp^{T}} \hat{\mathbb{C}} \mathbf{V}^{\perp}$ (notice that hypothesis (64) precludes the possibility of $\mathbf{V}^{\perp}$ being zero)

$$
\bar{G}=\frac{G}{\mathbf{V}^{{ }^{T}} \hat{\mathbb{C}} \mathbf{V}^{\perp}}=1+\frac{\mathbf{V}^{\perp^{T}} \hat{\mathbb{C}} \mathbf{V}^{\|}}{\mathbf{V}^{\perp T} \hat{\mathbb{C}} \mathbf{V}^{\perp}}
$$

Suppose now, for the sake of argument, that $\hat{\mathbb{C}}$ is also symmetric. Such being the case, the above equation can be legitimately rewritten as:

$$
\bar{G}=1+\cos \left(\mathbf{V}^{\perp}, \mathbf{V}^{\|}\right)_{\hat{\mathbb{C}}} \frac{\left\|\mathbf{V}^{\|}\right\|_{\hat{\mathbb{C}}}}{\left\|\mathbf{V}^{\perp}\right\|_{\hat{\mathbb{C}}}},
$$

where

$$
\cos \left(\mathbf{V}^{\perp}, \mathbf{V}^{\|}\right)_{\hat{\mathbb{C}}}=\frac{\left\langle\mathbf{V}^{\perp}, \mathbf{V}^{\|}\right\rangle_{\hat{\mathbb{C}}}}{\left\|\mathbf{V}^{\perp}\right\|_{\hat{\mathbb{C}}}\left\|\mathbf{V}^{\|}\right\|_{\hat{\mathbb{C}}}}
$$

In the above equation, $\langle\cdot, \cdot\rangle_{\widehat{\mathbb{C}}}$ symbolizes the inner product defined by $\hat{\mathbb{C}}$ (i.e., $\left.\langle\mathbf{x}, \mathbf{y}\rangle_{\hat{\mathbb{C}}}=\mathbf{x}^{\mathbf{T}} \hat{\mathbb{C}} \mathbf{y}\right)$, whereas $\|\cdot\|_{\hat{\mathbb{C}}}$ denotes the norm associated to such an inner product $\left(\|\mathbf{x}\|_{\hat{\mathbb{C}}}^{\mathbf{2}}=\langle\mathbf{x}, \mathbf{x}\rangle_{\hat{\mathbb{C}}}\right)$. From Eq.(94), it can be deduced that a sufficient (yet not necessary) condition for $\bar{G}>0$, and thus for $\boldsymbol{K}^{*}$ to be positive definite, is that

$$
\frac{\left\|\mathbf{V}^{\|}\right\|_{\hat{\mathbb{C}}}}{\left\|\mathbf{V}^{\perp}\right\|_{\hat{\mathbb{C}}}}<1
$$

for all nonzero $\mathbf{V} \in \operatorname{Range}\left(\hat{\mathbb{B}}^{*}\right)$, or equivalently ( $\left.\operatorname{setting} \mathbf{V}=\hat{\mathbb{B}}^{*} \mathbf{U}\right)$ :

$$
\frac{\left\|\hat{\boldsymbol{R}} \hat{\mathbb{B}}^{*} \mathbf{U}\right\|_{\hat{\mathbb{C}}}}{\left\|(\boldsymbol{I}-\hat{\boldsymbol{R}}) \hat{\mathbb{B}}^{*} \mathbf{U}\right\|_{\hat{\mathbb{C}}}}<1
$$

for all nonzero $\mathbf{U} \in \mathbb{R}^{\mathbf{n}_{\mathbf{u}}}$. 
Useful guidelines on how to choose $\mathcal{I}$ so as to make positive definite the Jacobian matrix $\boldsymbol{K}^{*}$ can be inferred from inequality (98). Firstly, given a fixed number of sampling points $p$, expression (98) indicates that such points should be selected so that the columns of the gappy strain basis matrix $\hat{\mathbb{B}}^{*}=$ $\mathcal{P}_{(\mathcal{I})} \mathbb{B}^{*}=\left[\hat{\mathbb{B}}_{1}^{*} \hat{\mathbb{B}}_{2}^{*} \ldots \hat{\mathbb{B}}_{n_{u}}^{*}\right]$ are, loosely speaking, "as orthogonal as possible" to Range $(\hat{\boldsymbol{R}})=\operatorname{Range}(\hat{\mathbf{\Psi}})$ - the column space of the gappy stress basis matrix $\hat{\boldsymbol{\Psi}}=\mathcal{P}_{(\mathcal{I})} \boldsymbol{\Psi}$. In so doing, the factor defined as

$$
f_{\hat{\mathbb{C}}}:=\frac{\sqrt{\sum_{i=1}^{n_{u}}\left\|\hat{\boldsymbol{R}} \hat{\mathbb{B}}_{i}^{*}\right\|_{\hat{\mathbb{C}}}^{2}}}{\sqrt{\sum_{i=1}^{n_{u}}\left\|(\boldsymbol{I}-\hat{\boldsymbol{R}}) \hat{\mathbb{B}}_{i}^{*}\right\|_{\hat{\mathbb{C}}}^{2}}},
$$

would diminish, and so would, consequently, the left-hand side of inequality Eq.(98). In practice, however, factor $f_{\widehat{\mathbb{C}}}$ cannot be used as a criterion for guiding the selection of sampling points, simply because it is defined in terms of the norm induced by $\hat{\mathbb{C}}$, and this matrix virtually changes at every time step and iteration. One has to be content to estimate this factor using other norm; for instance, employing the standard euclidean norm $\|\cdot\|$, one gets

$$
f_{\hat{\mathbb{C}}} \sim f_{F}:=\frac{\sqrt{\sum_{i=1}^{n_{u}}\left\|\hat{\boldsymbol{R}} \hat{\mathbb{B}}_{i}^{*}\right\|^{2}}}{\sqrt{\sum_{i=1}^{n_{u}}\left\|(\boldsymbol{I}-\hat{\boldsymbol{R}}) \hat{\mathbb{B}}_{i}^{*}\right\|^{2}}}=\frac{\left\|\hat{\boldsymbol{R}} \hat{\mathbb{B}}^{*}\right\|_{F}}{\left\|(\boldsymbol{I}-\hat{\boldsymbol{R}}) \hat{\mathbb{B}}^{*}\right\|_{F}},
$$

where $\|\cdot\|_{F}$ stands for the Frobenius norm.

Aside from seeking orthogonality between $\hat{\mathbb{B}}^{*}$ and $\hat{\boldsymbol{R}}$, expression (99) suggests that another way of lowering factor $f_{F}$ may be to reduce the ratio defined as

$$
\beta_{F}:=\frac{\|\hat{\boldsymbol{R}}\|_{F}}{\|\boldsymbol{I}-\hat{\boldsymbol{R}}\|_{F}} .
$$

Since $\hat{\boldsymbol{R}}$ and, consequently, $\boldsymbol{I}-\hat{\boldsymbol{R}}$ are matrices representing orthogonal projections, we have that $\|\hat{\boldsymbol{R}}\|_{F}=\sqrt{\operatorname{rank}(\hat{\boldsymbol{R}})}=\sqrt{n_{\sigma}}$ and $\|\boldsymbol{I}-\hat{\boldsymbol{R}}\|_{F}=\sqrt{p \cdot s-n_{\sigma}}$. Therefore,

$$
\beta_{F}=\sqrt{\frac{n_{\sigma}}{p \cdot s-n_{\sigma}}}
$$


Observation 6.2. From the above expression, thus, one can conclude that increasing the number of sampling points $p$ while keeping the number of stress modes $n_{\sigma}$ constant also contributes to reduce factor $f_{F}$ in Eq.(98), and, hence, to improve the spectral properties (positive defineteness) of the Jacobian matrix $\boldsymbol{K}^{*}$. Notice that this property is totally consistent with the fact that, in the limiting case of taking all Gauss points $\left(p=n_{g}\right)$, the reduced matrices $\hat{\boldsymbol{R}}$ and $\hat{\mathbb{B}}^{*}$ degenerate into their full order counterparts $\boldsymbol{R}$ and $\mathbb{B}^{*}$, for which the condition $\boldsymbol{R} \mathbb{B}^{*}=\mathbf{0}$ holds - they span subspaces that are mutually orthogonal-, hence making $f_{F}=f_{\widehat{\mathbb{C}}}=0$.

\section{Selection of sampling points}

The last theoretical issue to be discussed in the present work is the selection - among the full set of Gauss points of the underlying finite element mesh - of appropriate sampling or interpolation points. At the very least, the set of sampling indices $\mathcal{I}=\left\{i_{1}, i_{2} \ldots i_{p}\right\}$ must be chosen so that the gappy expanded basis matrix has full rank (see section 6.2):

$$
\operatorname{rank}\left(\hat{\mathbf{\Psi}}_{(\mathcal{I})}^{e x}\right)=\operatorname{rank}\left(\left[\hat{\mathbf{\Psi}}_{(\mathcal{I})} \hat{\mathbb{B}}_{(\mathcal{I})}^{*}\right]\right)=n_{\sigma}+n_{u}
$$

Any set of sampling indices fulfilling this necessary condition is said to be admissible.

\subsection{Optimality criteria}

\subsubsection{Accuracy}

As in any other model reduction problem, the overriding concern when choosing the sampling points is the accuracy of the approximation: we would like to position such points so that maximum similarity between the "highfidelity", finite element solution and its reduced-order counterpart is obtained. More specifically, since the output of interest in our BVP is the macroscopic stress response, the aim is to find the set of sampling points $\mathcal{I}$ that minimizes the following error estimate:

$$
E_{M, \sigma}(\Psi, \mathcal{I}):=\sqrt{\sum_{i=1}^{n_{s n p}}\left\|\boldsymbol{\sigma}_{M^{i}}-\boldsymbol{\sigma}_{M}^{* i}(\boldsymbol{\Psi}, \mathcal{I})\right\|^{2}}
$$

where $\boldsymbol{\sigma}_{\boldsymbol{M}^{i}}{ }^{i}=\boldsymbol{\sigma}_{\boldsymbol{M}}\left({ }^{t} \boldsymbol{\epsilon}_{\boldsymbol{M} k}{ }^{j}\right)$ denotes the finite element, macroscopic stress response corresponding to the the $k-t h\left(k=1,2 \ldots n_{\text {stp }}\right)$ time step of the 
"training" 16 strain trajectory ${ }^{t} \boldsymbol{\epsilon}_{\boldsymbol{M}^{j}}\left(j=1,2 \ldots n_{h s t}\right)$; and $\boldsymbol{\sigma}_{\boldsymbol{M}^{* i}}(\boldsymbol{\Psi}, \mathcal{I})$ its low-dimensional approximation.

Using the Cauchy-Schwarz inequality, and approximating the pertinent integrals by Gauss quadrature, we can write

$$
\begin{aligned}
E_{M, \sigma}{ }^{2} & \leq \frac{1}{V} \sum_{i=1}^{n_{\text {snp }}} \int_{\Omega}\left\|\boldsymbol{\sigma}^{i}(\mathbf{x})-\boldsymbol{\sigma}^{* i}(\mathbf{x} ; \boldsymbol{\Psi}, \mathcal{I})\right\|^{2} d \Omega \\
& \approx \frac{1}{V} \sum_{i=1}^{n_{\text {snp }}} \sum_{g=1}^{n_{g}}\left\|\sqrt{w_{g}} \boldsymbol{\sigma}^{i}\left(\mathbf{x}_{g}\right)-\sqrt{w_{g}} \boldsymbol{\sigma}^{* i}\left(\mathbf{x}_{g} ; \boldsymbol{\Psi}, \mathcal{I}\right)\right\|^{2} \\
& =\frac{1}{V} \sum_{i=1}^{n_{\text {snp }}}\left\|\boldsymbol{\Sigma}^{i}-\boldsymbol{\Sigma}^{* i}(\boldsymbol{\Psi}, \mathcal{I})\right\|^{2} 2=\frac{1}{V}\left\|\boldsymbol{X}-\boldsymbol{X}^{*}(\boldsymbol{\Psi}, \mathcal{I})\right\|_{F}^{2}
\end{aligned}
$$

where $\boldsymbol{X}=\left[\begin{array}{llll}\boldsymbol{\Sigma}^{1} & \boldsymbol{\Sigma}^{2} & \cdots & \boldsymbol{\Sigma}^{n_{s n p}}\end{array}\right]$ and $\boldsymbol{X}^{*}(\boldsymbol{\Psi}, \mathcal{I})=\boldsymbol{R}_{(\mathcal{I})}\left(\mathcal{P}_{(\mathcal{I})} \boldsymbol{X}\right)$. The error estimate for the macroscopic stresses defined in Eq.(104) is, thus, bounded above by the Frobenius norm of the difference between the (weighted) stress snapshot matrix $\boldsymbol{X}$ and its oblique projection, $\boldsymbol{X}^{*}$, onto $\operatorname{Range}(\boldsymbol{\Psi})$. This bound, hereafter designated by $e_{\sigma}$, admits the following decomposition ${ }^{17}$

$$
e_{\sigma}^{2}=\frac{1}{V}\left\|\boldsymbol{X}-\boldsymbol{X}^{* \perp}(\boldsymbol{\Psi})\right\|_{F}^{2}+\frac{1}{V}\left\|\boldsymbol{X}^{* \perp}(\boldsymbol{\Psi})-\boldsymbol{X}^{*}(\boldsymbol{\Psi}, \mathcal{I})\right\|_{F}^{2},
$$

$\boldsymbol{X}^{* \perp}$ being the orthogonal projection of $\boldsymbol{X}$ onto the range of $\boldsymbol{\Psi}$, i.e., $\boldsymbol{X}^{* \perp}=$ $\boldsymbol{\Psi} \boldsymbol{\Psi}^{T} \boldsymbol{X}$. Note that the first term of the right-hand side of Eq.(106) only depends on the stress basis matrix, but not on the employed sampling indices; it provides, thus, an estimate of the stress truncation error. The term that actually measures the quality, in terms of accuracy, of a given set of admissible sampling points is the second one -it provides an (a priori) estimate of the stress reconstruction error. We shall denote this term by $e_{\sigma}^{r e c}$ :

$$
e_{\sigma}^{r e c}:=\frac{1}{V}\left\|\boldsymbol{X}^{* \perp}(\boldsymbol{\Psi})-\boldsymbol{X}^{*}(\boldsymbol{\Psi}, \mathcal{I})\right\|_{F} .
$$

For this reason - and also because the cost of evaluating expression Eq.(107)

\footnotetext{
${ }^{16}$ The term "training", which, incidentally, is borrowed from the neural network literature [38], is used throughout the text to refer to the offline generation of snapshots.

${ }^{17}$ This decomposition follows easily from the Pythagorean theorem (just notice that $\left.\boldsymbol{\Psi}^{T}\left(\boldsymbol{X}-\boldsymbol{X}^{* \perp}\right)=\mathbf{0}\right)$.
} 
is independent of the number of Gauss points ${ }^{18}$, and therefore significantly lower than in the case of the original error estimate $E_{M, \sigma}$, we shall use in what follows $e_{\sigma}^{r e c}$ as error estimator for guiding the selection of sampling points.

\subsubsection{Spectral properties}

Yet the optimality of a given set of sampling points cannot be measured only in terms of accuracy of the approximation. As demonstrated in Section 6.4, the number and particular placement of such points influence also the spectral properties (positive definiteness) of the Jacobian matrix of the equilibrium equation, and therefore, the convergence characteristics of the accompanying Newton-Raphson algorithm. We saw that, to preserve the positive definiteness of the full-order Jacobian matrix, one should strive to choose the sampling indices $\mathcal{I}$ so as to make the factor - defined previously in Eq.(100)—:

$$
f_{F}\left(\boldsymbol{\Psi}, \mathbb{B}^{*}, \mathcal{I}\right)=\frac{\left\|\hat{\boldsymbol{R}}_{(\mathcal{I})} \hat{\mathbb{B}}_{(\mathcal{I})}^{*}\right\|_{F}}{\left\|\left(\boldsymbol{I}-\hat{\boldsymbol{R}}_{(\mathcal{I})}\right) \hat{\mathbb{B}}_{(\mathcal{I})}^{*}\right\|_{F}}
$$

as small as possible.

\subsection{Optimization approach: basic and stabilizing sampling points}

Unfortunately, the minimization of the approximation error represented by expression Eq.(107) and the minimization of Eq.(108) are in general conflicting goals. For instance, numerical experiments show that when the selection is driven exclusively by accuracy considerations, the resulting Jacobian matrix becomes indefinite at certain states of deformation - especially when inelastic deformations are severe-, leading occasionally to convergence failures. These goals must be therefore balanced in order to arrive at an accurate and at the same time robust solution scheme.

To accomodate these conflicting requirements, we propose here a heuristic strategy that basically consists in treating the minimization of Eq.(107) and

\footnotetext{
${ }^{18}$ Indeed, since $\boldsymbol{\Psi}$ is column-wise orthogonal, minimizing Eq.(107) is equivalent to minimizing the difference between the coefficients of the respective projections. The number of coefficients is equal to the number of snapshots, which is normally much lower than the number of Gauss points.
} 
Eq.(108) as two separated, sequential problems - in the spirit of the socalled "greedy" optimization algorithms ${ }^{19}$ [44]. The set of sampling points is assumed to be divided into two disjoint subsets $\mathcal{I}_{\sigma}$ and $\mathcal{I}_{B}$ :

$$
\mathcal{I}=\mathcal{I}_{\sigma} \cup \mathcal{I}_{B}
$$

The first subset $\mathcal{I}_{\sigma}=\left\{i_{1}, i_{2}, \cdots i_{p_{\sigma}}\right\}$ is obtained as the minimizer of the error estimation given in Eq.(107), viz.:

$$
\mathcal{I}_{\sigma}=\arg \min _{\mathcal{K} \subseteq\left\{1,2 \ldots n_{g}\right\}} e_{\sigma}^{r e c}(\Psi, \mathcal{K}) .
$$

Once the set $\mathcal{I}_{\sigma}$ is determined, the remaining sampling indices $\mathcal{I}_{B}=\left\{j_{1}, j_{2} \cdots j_{p_{B}}\right\}$ $\left(p_{\sigma}+p_{B}=p\right)$ are calculated as

$$
\mathcal{I}_{B}=\arg \min _{\mathcal{K} \subseteq\left\{1,2 \ldots n_{g}\right\}} f_{F}\left(\Psi, \mathbb{B}^{*}, \mathcal{I}_{\sigma} \cup \mathcal{K}\right) .
$$

Remark 7.1. It must be noted here that the minimization problem represented by Eq.(110) is in essence the same problem addressed in (standard) interpolatory-based, model reduction approaches for determining, given a set of empirical basis functions, the optimal location of associated interpolations points. For this reason, we shall refer to the set of points arising from solving this minimization problem as the standard or basic sampling points - these are the Best Interpolation Points of Nguyen et al. [54], or the "magic points" of Maday et al. [46].. By contrast, the necessity of introducing points that attempt to solve problem (111) is a consequence of expanding the stress approximation space in the first place - the main innovative feature of our approach-, and it is therefore not present in other model reduction strategies. We shall call $\left\{\boldsymbol{x}_{\mathcal{I}_{B}(1)}, \boldsymbol{x}_{\mathcal{I}_{B}(2)} \ldots \boldsymbol{x}_{\mathcal{I}_{B}\left(p_{B}\right)}\right\}$ the set of stabilizing sampling points.

The number of basic sampling points must satisfy the necessary condition $p_{\sigma} \geq n_{\sigma} / s$. In general, taking $p_{\sigma}=n_{\sigma}$ suffices to ensure highly satisfactory approximations. How many, on the other hand, stabilizing sampling points have to be added to safely render positive definite the Jacobian matrix for at least a representative range of macroscopic state deformations - is a

\footnotetext{
${ }^{19} \mathrm{~A}$ greedy method is any algorithm that solves the problem by making the locally optimal choice at each step with the hope of finding the global optimum.
} 
question that can only be answered empirically. In the examples presented in the next section, it has been found that a conservative answer is to use as many stabilizing sampling points as displacement basis modes $\left(p_{B}=n_{u}\right)$.

To deal with the discrete minimization problem (110), we have used here the Hierarchical Interpolation Points (HPI) method proposed by Nguyen et al. [54]. The essence of this method is to construct, in a greedy fashion, the set of indices by solving a sequence of one-dimensional minimization problems. The minimization problem (111) is also addressed using a heuristic based on the greedy paradigm. In particular, the $k-t h\left(k=1,2 \ldots p_{B}\right)$ index is selected by solving the following, one-dimensional minimization problem:

$$
\begin{gathered}
\mathcal{I}_{B}(k)=\arg \min _{j \in\left\{1,2 \ldots n_{g}\right\}} f_{F}\left(\Psi, \mathbb{B}^{*}, \mathcal{K}(j)\right) \\
\mathcal{K}=\mathcal{I}_{\sigma} \cup\left\{\mathcal{I}_{B}(1), \mathcal{I}_{B}(2) \cdots \mathcal{I}_{B}(k-1), j\right\} .
\end{gathered}
$$

\section{Summary}

Lastly, for the reader's convenience and easy reference, the online reducedorder problem, along with the offline steps that leads to the the hyperreduced operators appearing in the online problem, are summarized in Boxes 8.1 and 8.2 .

\section{Numerical results}

This section is intended to illustrate the performance and assess the efficiency of the proposed model reduction strategy in solving the fine scale BVP corresponding to a porous metal material under plane strain conditions.

\subsection{Microstructure description}

The voids are elliptical in shape (with eccentricity equal to 0.3 ), randomly distributed (with porosity equal to 0.3 ), and have aligned major axes ranging in length - according to the cumulative probability distribution displayed in figure 3.b- from 0.2 to $1.5 \mathrm{~mm}$. The mechanical behavior of the metal matrix is modeled by a rate-independent, Von Mises elastoplastic model endowed with the following non-linear, isotropic hardening saturation law (consult Ref. [61] for details on the implementation of this elastoplastic model):

$$
\sigma_{u}(\alpha)=\sigma_{0}+\bar{H} \alpha+\left(\sigma_{\infty}-\sigma_{0}\right)(1-\exp (-\alpha \delta)) .
$$


1. Compute FE displacement fluctuations and stress snaphots for representative, input macro-strain histories. Apply — see Appendix B - the elastic/inelastic POD to the resulting snapshot matrices to obtain the displacement fluctuation and stress basis matrices $\left(\boldsymbol{\Phi} \in \mathbb{R}^{n \cdot d \times n_{u}}\right.$ and $\boldsymbol{\Psi} \in \mathbb{R}^{n_{g} \cdot s \times n_{\sigma}}$, respectively).

2. Calculate the weighted matrix of fluctuating strain modes $\mathbb{B}^{*} \in$ $\mathbb{R}^{n_{g} \cdot s \times n_{\sigma}}$ using Eqs. (26) and (47).

3. Select a set $\mathcal{I}$ of sampling indices optimal for the basis matrices $\boldsymbol{\Psi}$ and $\mathbb{B}^{*}$ following the procedure sketched in Section 7 .

4. Finally, using $\Psi, \mathbb{B}^{*}$ and $\mathcal{I}$, construct the hyperreduced-order matrices $\hat{\mathbb{B}}^{* *} \in \mathbb{R}^{p \cdot s \times n_{u}}$ and $\mathbb{T} \in \mathbb{R}^{s \times p \cdot s}$; the expressions for these matrices read:

$$
\hat{\mathbb{B}}^{* *}=(\boldsymbol{I}-\hat{\boldsymbol{R}}) \hat{\mathbb{B}}^{*}=\left(\boldsymbol{I}-\mathcal{P}_{(\mathcal{I})} \boldsymbol{R}\right)\left(\mathcal{P}_{(\mathcal{I})} \mathbb{B}^{*}\right)
$$

and

$$
\mathbb{T}:=\frac{1}{V}\left[\begin{array}{llll}
\sqrt{w_{1}} \boldsymbol{I} & \sqrt{w_{2}} \boldsymbol{I} & \cdots & \sqrt{w_{n_{g}}} \boldsymbol{I}
\end{array}\right] \boldsymbol{R}
$$

where $\boldsymbol{R}=\boldsymbol{\Psi}\left(\hat{\boldsymbol{\Psi}}^{T} \hat{\boldsymbol{\Psi}}\right)^{-1} \hat{\boldsymbol{\Psi}}^{T}$ and $\hat{\boldsymbol{\Psi}}=\mathcal{P}_{(\mathcal{I})} \boldsymbol{\Psi}$.

Box 8.1: Offline stage. Pre-computation of reduced basis and hyperreduced operators.

Here, $\sigma_{u}$ stands for the yield stress, $\alpha \geq 0$ denotes the equivalent plastic strain; and $\sigma_{0}=75.0 \mathrm{MPa}, \sigma_{\infty}=100.0 \mathrm{MPa}, \delta=2500.0$ and $\bar{H}=5000$ $M P a$ are material constants. The Young's modulus and Poisson's coefficient, on the other hand, are equal to $E_{m}=75 G P a$ and $\nu_{m}=0.3$, respectively (these material constants corresponds approximately to Aluminum).

\subsection{RVE and finite element discretization}

The size of the RVE was determined by conducting finite element analyses on square domains of increasing size subject to vanishing displacement fluctuations boundary conditions. It was found that the macroscopic stress responses calculated under representative macroscopic strain paths (stretching along the longitudinal and transversal directions, and shearing) of all samples above $20 \times 20 \mathrm{~mm}^{2}$ were practically indistinguishable. This fact indicates that any subvolume of $20 \times 20 \mathrm{~mm}^{2}$ (or greater) can be considered as a Representative Volume Element (RVE) of the porous material under study. 
1. Initial data: $\boldsymbol{U}_{n}^{*} \in \mathbb{R}^{n_{u}}$ (reduced vector of displacement fluctuations at $t_{n}$ ), $\boldsymbol{\epsilon}_{\boldsymbol{M}_{n}}$ (macroscopic strain vector at $t_{n}$ ), and $\left\{\boldsymbol{\xi}_{n}\left(\mathbf{x}_{\mathcal{I}_{1}}\right), \boldsymbol{\xi}_{n}\left(\mathbf{x}_{\mathcal{I}_{2}}\right), \ldots \boldsymbol{\xi}_{n}\left(\mathbf{x}_{\mathcal{I}_{p}}\right)\right\}$ (internal variables at $t_{n}$ at the selected sampling points).

2. Input data: $\boldsymbol{\epsilon}_{\boldsymbol{M} n+1}$ (macroscopic strain vector at $t_{n+1}$ )

3. Given the above initial and input data, find $\boldsymbol{U}_{n+1}^{*} \in \mathbb{R}^{n_{u}}$ such that

$$
\hat{\mathbb{B}}^{*^{T}} \hat{\boldsymbol{\Sigma}}\left(\boldsymbol{\epsilon}_{\boldsymbol{M}_{n+1}}, \boldsymbol{U}_{n+1}^{*}\right)=\mathbf{0},
$$

where

$$
\hat{\boldsymbol{\Sigma}}=\left[\begin{array}{lllll}
\sqrt{w_{\mathcal{I}_{1}}} \boldsymbol{\sigma}^{T}\left(\mathbf{x}_{\mathcal{I}_{1}}, \cdot\right) & \sqrt{w_{\mathcal{I}_{2}}} \boldsymbol{\sigma}^{T}\left(\mathbf{x}_{\mathcal{I}_{2}}, \cdot\right) & \cdots & w_{\mathcal{I}_{p}} \boldsymbol{\sigma}^{T}\left(\mathbf{x}_{\mathcal{I}_{p}}, \cdot\right)
\end{array}\right]^{T}
$$

(here, $\boldsymbol{\sigma}\left(\mathbf{x}_{\mathcal{I}_{i}}, \cdot\right)$ denotes the stress vector evaluated at the $i-t h$ sampling point through the corresponding constitutive equation).

4. Output data: Once Eq.(116) has been solved for $\boldsymbol{U}_{n+1}^{*}$, update the macroscopic stress vector as

$$
\left.\boldsymbol{\sigma}_{M}\right|_{n+1}=\mathbb{T} \hat{\Sigma}\left(\epsilon_{M_{n+1}}, \boldsymbol{U}_{n+1}^{*}\right) .
$$

Box 8.2: Online stage (solution of the hyperreduced-order RVE equilibrium problem for given macroscopic strains).

The finite element discretization corresponding to the particular 20x20 $\mathrm{mm}^{2}$ RVE employed in the ensuing simulations is shown in figure 3.a. The number of (four-node bilinear) elements is $n_{e}=9746$, and the number of nodes $n=11825$. The employed quadrature formula, on the other hand, is the standard $2 \times 2$ Gauss rule, the total number of Gauss points amounting thus to $n_{g}=4 n_{e}=38984$. To overcome incompressibility issues while maintaining the displacement-based formulation presented in the preceding sections, the commonly known as "B-bar" approach is adopted ${ }^{20}$. The constitutive differential equations are integrated in time using the classical (fully

\footnotetext{
${ }^{20}$ This means that, in this case, the reduced "B-matrix" $\boldsymbol{B}^{*}(\mathbf{x})$ appearing in the formulation of the HP-ROM is not constructed using the gradients of the shape functions, as indicated by Eq.(23), but rather using the modified "B-matrix" emanating from the three-field Hu-Washizu variational principle [61]
} 


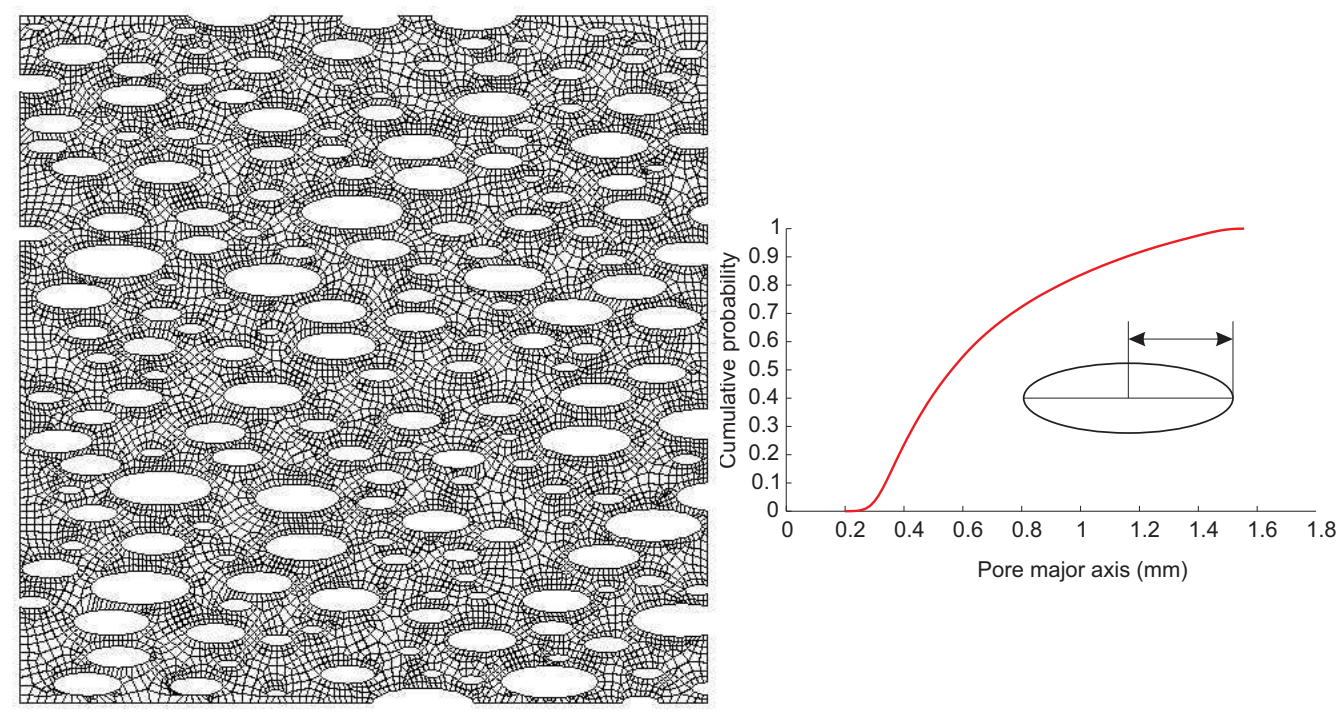

Figure 3: a) Finite element mesh of the RVE corresponding to the porous metal material. b) Cumulative probability distribution followed by the length of the pore major axes.

implicit) backward-Euler scheme.

\subsection{Sampling of parameter space}

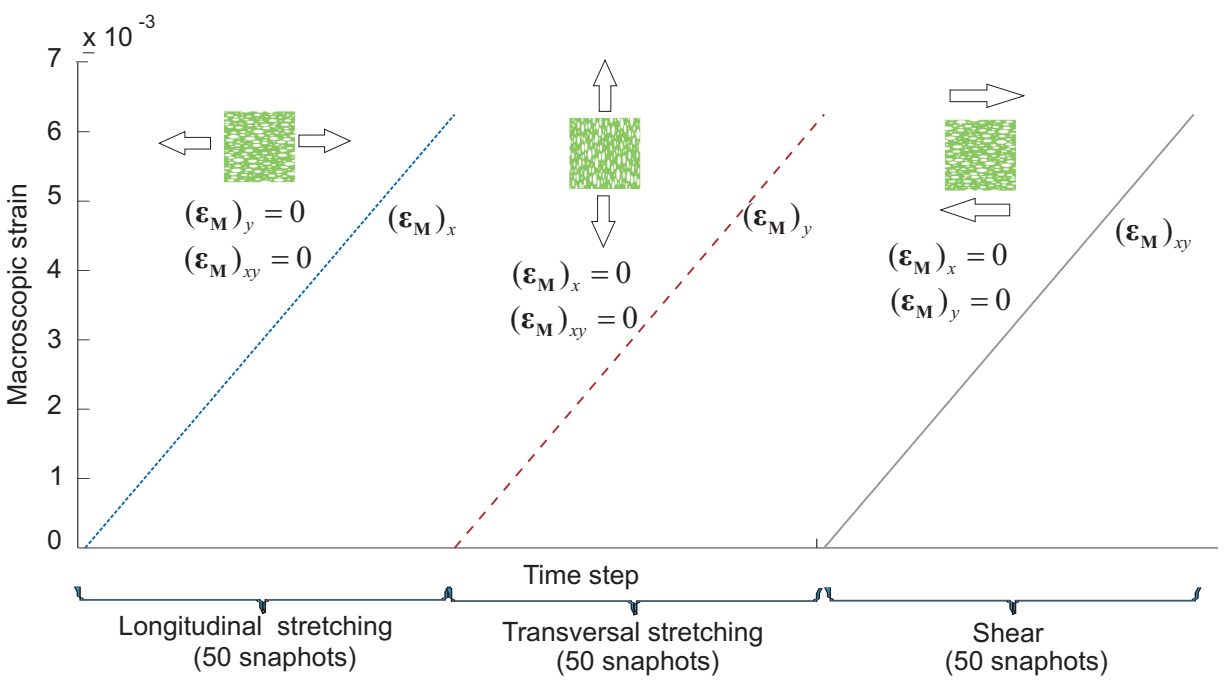

Figure 4: Macro-strain trajectories used for generating the displacement and stress snapshots. 
The first step in the process of constructing the reduced basis is the sampling of the input parameter space; we saw in Section 3.1 that, in the fine-scale BVP, this process amounts to select representative macroscopic strain histories. The three macroscopic strain histories $\left(n_{\text {hst }}=3\right)$ used in the case under study are depicted in figure 4. In each of these strain trajectories, one of the (independent) strain components follows a linear ascending path while the magnitude of the other two components is set to zero. The time domain for each strain history is discretized into $n_{s t p}=50$ equally spaced steps, resulting in a total number of $n_{s n p}=n_{h s t} \cdot n_{s t p}=150$ snapshots.

Remark 9.1. The task of sampling the input parameter space is somehow akin to the experimental process whereby material parameters of standard phenomenological models are calibrated in a laboratory. In this analogy, the $R V E$ plays the role of the corresponding experimental specimen, whereas the macro-strain training trajectories represent the loading paths of the pertinent calibration tests. Hindsight and elementary physical considerations can therefore aid in restricting the number of strain histories (and hence of snapshots) necessary to characterize the response. For instance, if the behavior of the materials that compose the RVE is governed by rate-independent constitutive models (as in the case at hand), we know beforehand that it is not necessary to study the response under varying rates of deformation.

Strategies for efficiently sampling the input parameter space in general model reduction contexts can be found in Refs. [12, 13, 15, 42].

\subsection{Dimensionality reduction: a priori error analysis}

The finite element displacement fluctuation and stress fields computed at each time step of the input strain trajectories shown above are multiplied by their corresponding weighting matrices ( $\overline{\mathbf{M}}$ and $\boldsymbol{W}^{1 / 2}$ ) and stored, in the snapshot matrices $\overline{\boldsymbol{X}}_{u} \in \mathbb{R}^{n \cdot d \times n_{\text {snp }}}(n \cdot d=11825 \cdot 2=23650)$ and $\boldsymbol{X} \in \mathbb{R}^{n_{g} \cdot s \times n_{s n p}}\left(n_{g} \cdot s=38984 \cdot 4=155936\right)$, respectively. Then, these matrices are subjected to the SVD-based, elastic/inelastic dimensionality reduction process sketched in Section 3.2.1 — and described more in detail in Appendix B - in order to generate an optimal set of basis vectors for both the displacements fluctuation and stress solution spaces.

To elucidate which of these basis vectors constitute the "essential" modes of the response, we plot in Figure 5 the dimensionless POD truncation error estimates defined, for the displacement fluctuations, as: 


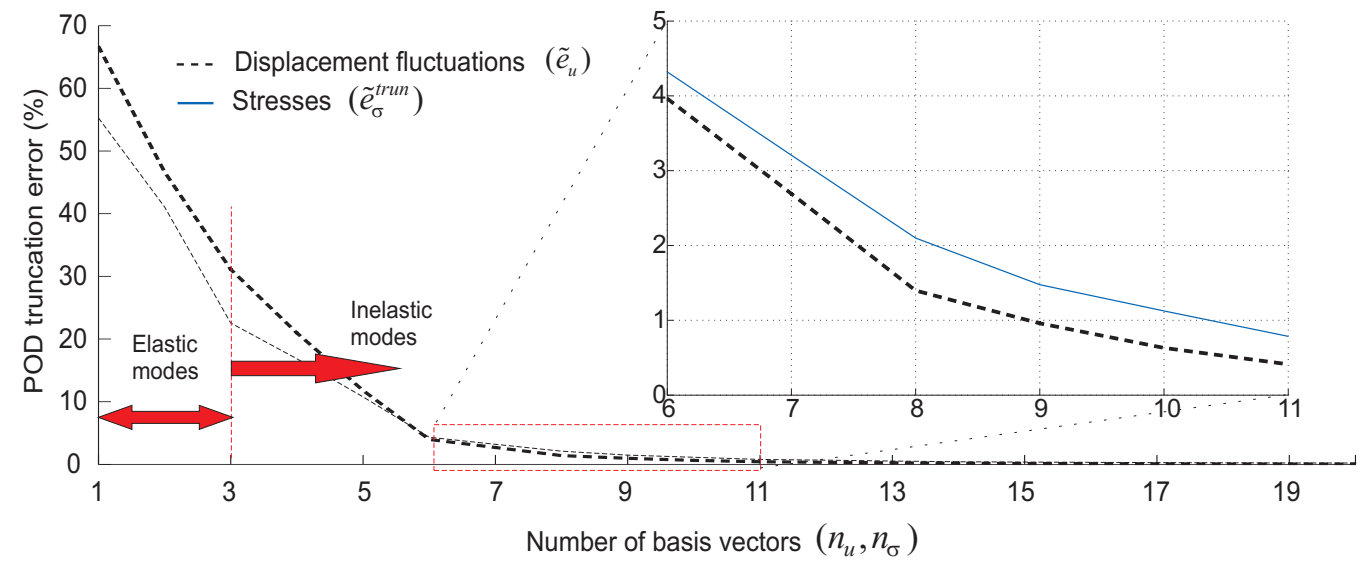

Figure 5: POD truncation error estimates $\tilde{e}_{u}$ (for the displacement fluctuations, see Eq.(119)) and $\tilde{e}_{\sigma}^{\text {trun }}$ (for the stresses, see Eq.(120)) versus number of basis vectors employed in the approximation $\left(n_{u}\right.$ and $n_{\sigma}$, respectively). The portion between 6 and 11 modes is shown in magnified form.

$$
\tilde{e}_{u}\left(n_{u}\right):=\frac{\left\|\overline{\boldsymbol{X}}_{u}-\overline{\boldsymbol{X}}_{u}^{*}\left(n_{u}\right)\right\|_{F}}{\left\|\overline{\boldsymbol{X}}_{u}\right\|_{F}}
$$

and for the stresses:

$$
\tilde{e}_{\sigma}^{\text {trun }}\left(n_{\sigma}\right):=\frac{\left\|\boldsymbol{X}-\boldsymbol{X}^{* \perp}\left(n_{\sigma}\right)\right\|_{F}}{\|\boldsymbol{X}\|_{F}}
$$

$\overline{\boldsymbol{X}}_{u}^{*}\left(n_{u}\right)$ and $\boldsymbol{X}^{* \perp}\left(n_{\sigma}\right)$ being the orthogonal projection of $\overline{\boldsymbol{X}}_{u}$ and $\boldsymbol{X}$ onto the span of the first $n_{u}$ and $n_{\sigma}$ basis vectors, respectively. It can be observed in Figure 5 that both error measures decrease monotonically with increasing order of truncation - this is a mere consequence of the optimality properties of the SVD - , and at approximately the same rate; the decay is more pronounced from 1 to 6 modes, and becomes more gradual thereafter, tending asymptotically to zero as the number of modes increases. The truncation error for both stresses and displacement fluctuations at $n_{\sigma}=n_{u}=6$ is around 5\%. In terms of dimensionality reduction, this means that the data contained in the snapshot matrices can be "compressed" to a factor of $\left(n_{u} / n_{\text {snp }}\right) \cdot 100=(6 / 150) \cdot 100=4 \%$ and still retain $95 \%$ of the information - the essential information. The first 6 basis functions (3 elastic and 3 inelastic) for both stresses and displacement fluctuations, therefore, are to be regarded as essential modes in the characterization of the mechanical 


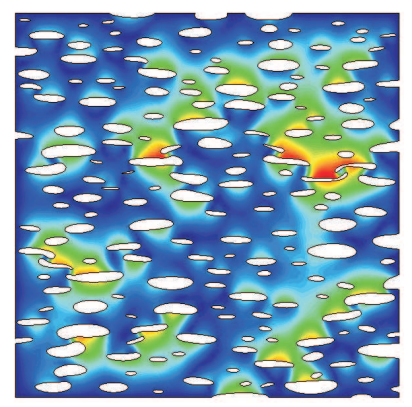

a) 1 st basis

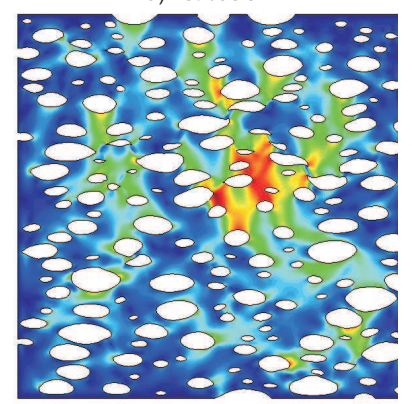

d) 4 th basis

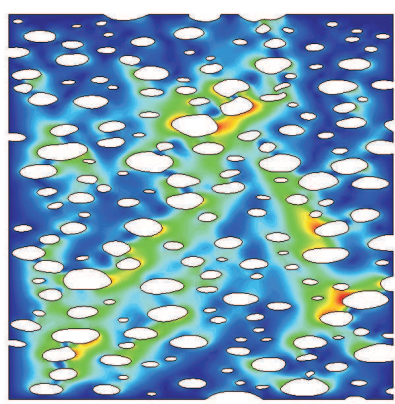

b) 2nd basis

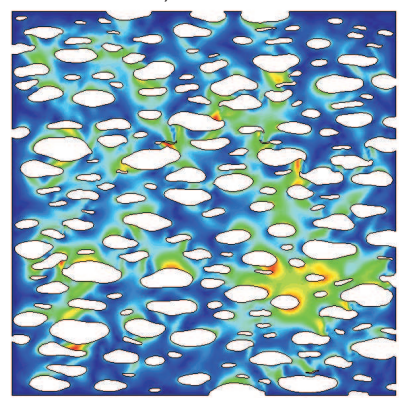

e) 5 th basis

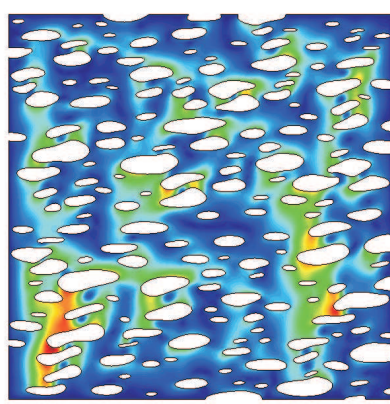

c) 3rd basis

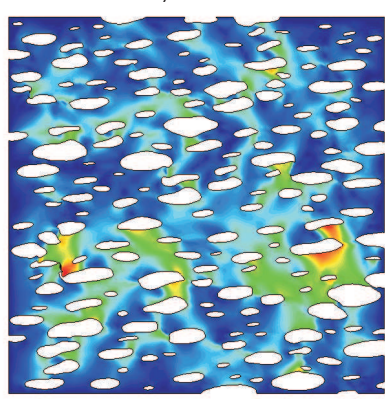

f) 6 th basis

Figure 6: Contour plots of the euclidean norm of the first 6 displacement fluctuations modes $\left(\left\|\boldsymbol{\Phi}_{i}\right\|, i=1,2 \ldots 5\right)$. Deformed shapes are scaled up by a factor of 15 .

response of the concerned RVE. By way of illustration, we plot in Figure 6 the contour plots of the euclidean norm of such 6 essential displacement fluctuations modes $\left(\left\|\boldsymbol{\Phi}_{i}\right\|, i=1,2 \ldots 6\right)$.

\subsection{Sampling points}

\subsubsection{Basic sampling points}

Once the stress and displacement fluctuation basis vectors have been determined, the next offline step consists in the selection - among the full set of finite element Gauss points - of an optimal set of sampling points. Following the strategy described in Section 7.2, we carry out such a selection by first computing the location of what we have called basic sampling points $\left\{\mathbf{x}_{\mathcal{I}_{\sigma}(1)}, \mathbf{x}_{\mathcal{I}_{\sigma}(2)} \ldots \mathbf{x}_{\mathcal{I}_{\sigma}\left(p_{\sigma}\right)}\right\}$. To assess the efficiency of the employed Hierarchical Interpolation Points Method, abbreviated HIPM, we plot in Figure 7 the estimates for both the POD truncation (shown previously in Figure 5) and total stress error versus the number of stress modes $n_{\sigma}$ (in using this algorithm, it is assumed that $\left.p_{\sigma}=n_{\sigma}\right)$. The total stress error estimate is 


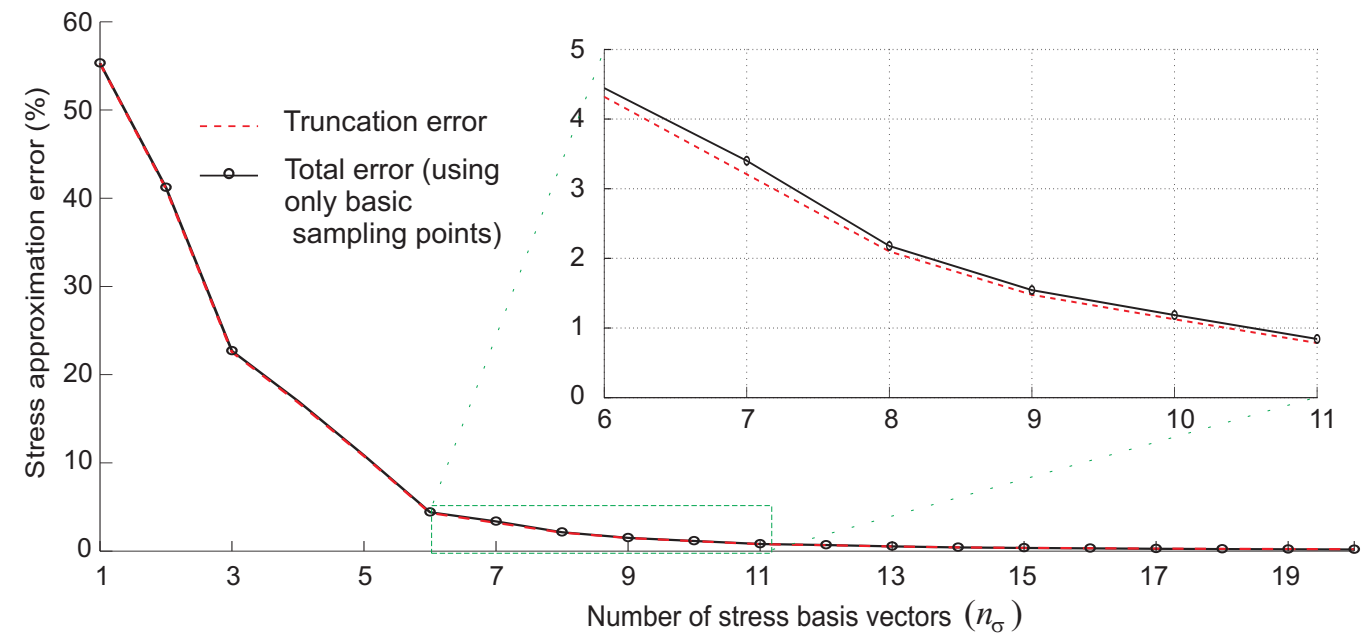

Figure 7: Estimates for the POD truncation ( $\tilde{e}_{\sigma}^{\text {trun }}$, see Eq.(120)) and total ( $\tilde{e}_{\sigma}$, see Eq.(121)) stress error versus number of basis vectors employed in the approximation $\left(n_{\sigma}\right)$. The total error estimate is computed using only the set of basic sampling points $\left(\tilde{e}_{\sigma}=\right.$ $\tilde{e}_{\sigma}\left(n_{\sigma}, \mathcal{I}_{\sigma}\right)$, with $\left.p_{\sigma}=n_{\sigma}\right)$. The portion between 6 and 11 modes is shown in magnified form.

defined as

$$
\tilde{e}_{\sigma}\left(n_{\sigma}, \mathcal{I}_{\sigma}\right):=\frac{\left\|\boldsymbol{X}-\boldsymbol{X}^{*}\left(n_{\sigma}, \mathcal{I}_{\sigma}\right)\right\|_{F}}{\|\boldsymbol{X}\|_{F}}
$$

where $\boldsymbol{X}^{*}\left(n_{\sigma}, \mathcal{I}_{\sigma}\right)$ denotes the oblique projection (calculated using sampling points $\left.\mathcal{I}_{\sigma}\right)$ of $\boldsymbol{X}$ onto the span of the first $n_{\sigma}$ basis vectors $\left(\boldsymbol{\Psi}_{1}, \boldsymbol{\Psi}_{2} \ldots \boldsymbol{\Psi}_{n_{\sigma}}\right)$. It can be appreciated in Figure 7 that both the total error and the truncation error curves are practically coincident, a fact that indicates that the contribution of the reconstruction error:

$$
\tilde{e}_{\sigma}^{r e c}=\sqrt{\tilde{e}_{\sigma}^{2}-\tilde{e}_{\sigma}^{\operatorname{trun2} 2}}=\frac{\left\|\boldsymbol{X}^{* \perp}\left(n_{\sigma}\right)-\boldsymbol{X}^{*}\left(n_{\sigma}, \mathcal{I}_{\sigma}\right)\right\|_{F}}{\|\boldsymbol{X}\|_{F}}
$$

( the error introduced as a result of using only $p_{\sigma}$ sampling points instead of the entire set of finite element Gauss points, see Section 7.1.1) is negligible in comparison to the discrepancies due to truncation of the POD basis. For $n_{\sigma}=p_{\sigma}=6$, for instance, the reconstruction error is less than $3 \%$ of the total stress error. In view of these results, it becomes clear that further refinements in the algorithm for selecting the basic sampling points are in principle not necessary: the employed HIPM optimization algorithm, however 
heuristic, satisfactorily fulfills this purpose. If one wishes to lower the stress approximation error, it is far more effective to simply increase the level of truncation.

\subsubsection{Stabilizing sampling points}
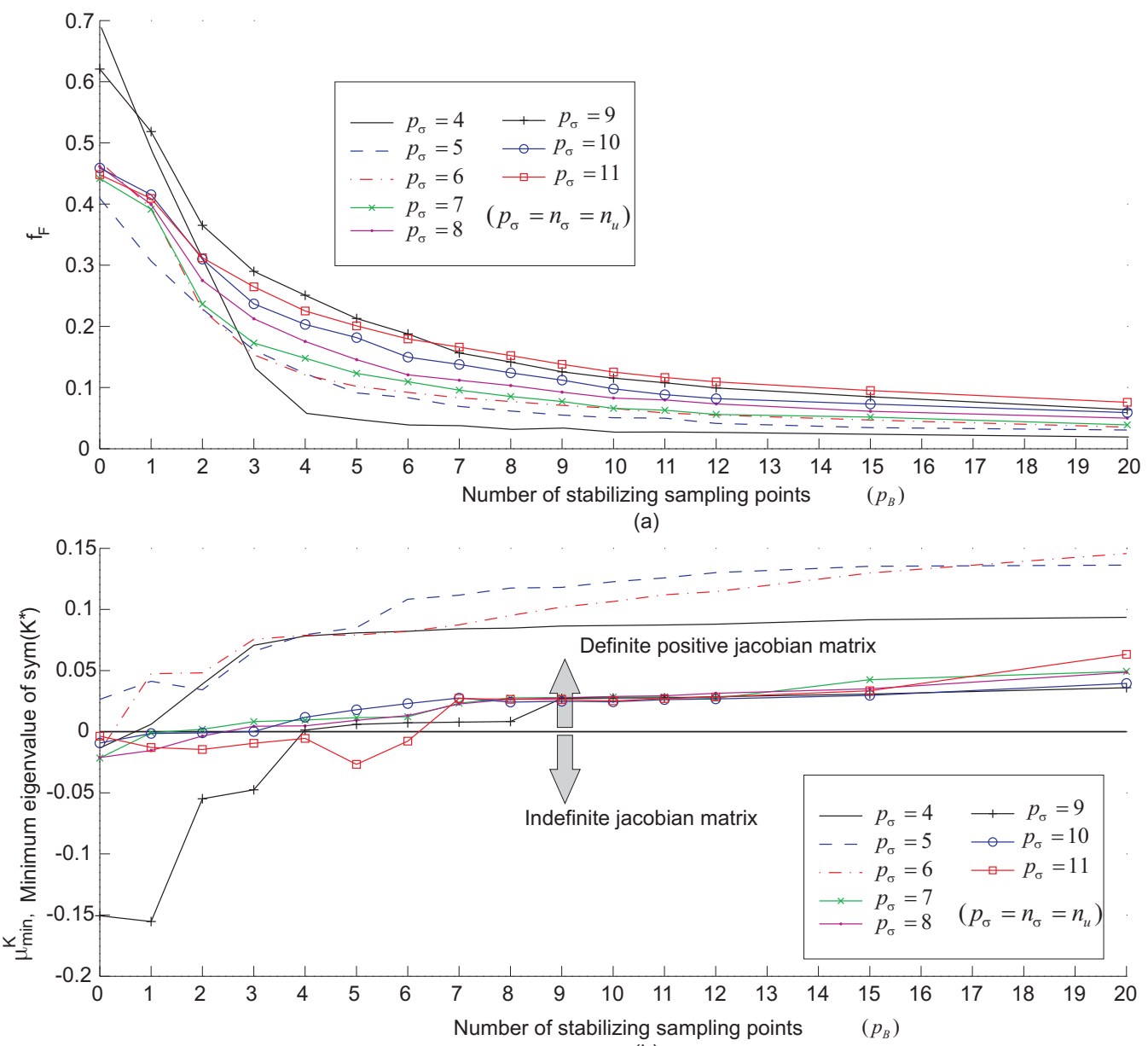

(b)

Figure 8: a) Factor $f_{F}$ (defined in Eq.(99)) versus number of stabilizing sampling points $p_{B}$ for varying numbers of basic sampling points $p_{\sigma}$ (with $p_{\sigma}=n_{\sigma}=n_{u}$ ). b) Minimum eigenvalue $\mu_{\min }^{K}$ (over all time steps and iterations for each $p_{\sigma}$ ) of the symmetric part of the reduced-order Jacobian matrix $\boldsymbol{K}^{*}$ versus number of stabilizing sampling points $p_{B}$.

Concerning what we have termed "stabilizing sampling points", Figure 8.a contains the graphs, for varying levels of truncation, of factor $f_{F}$ defined 
in Eq.(100) as a function of the number of stabilizing sampling points $p_{B}$. To study the influence of including such points on the spectral properties positive defineteness - of the stiffness matrix, these graphs are accompanied, see figure 8.b, by the plots of the minimum eigenvalue $\mu_{\min }^{K}$ (over all time steps and iterations for each case) of the symmetric part of the reduced-order Jacobian matrix $\boldsymbol{K}^{*}$ versus $p_{B}$. It can be seen that $f_{F}$ decreases monotonically as the number of stabilizing sampling points increases, and such a decrease is reflected, as theoretically anticipated in Section 6.4.1, in the improvement of the spectral properties of the reduced-order Jacobian matrix (higher $\mu_{\min }^{K}$ as $p_{B}$ raises). For clarity, the minimum number of stabilizing sampling points required, for each level of truncation, to render positive definite $\boldsymbol{K}^{*}$ is plotted in Figure 9. From this plot, it can be gleaned that, roughly, the higher

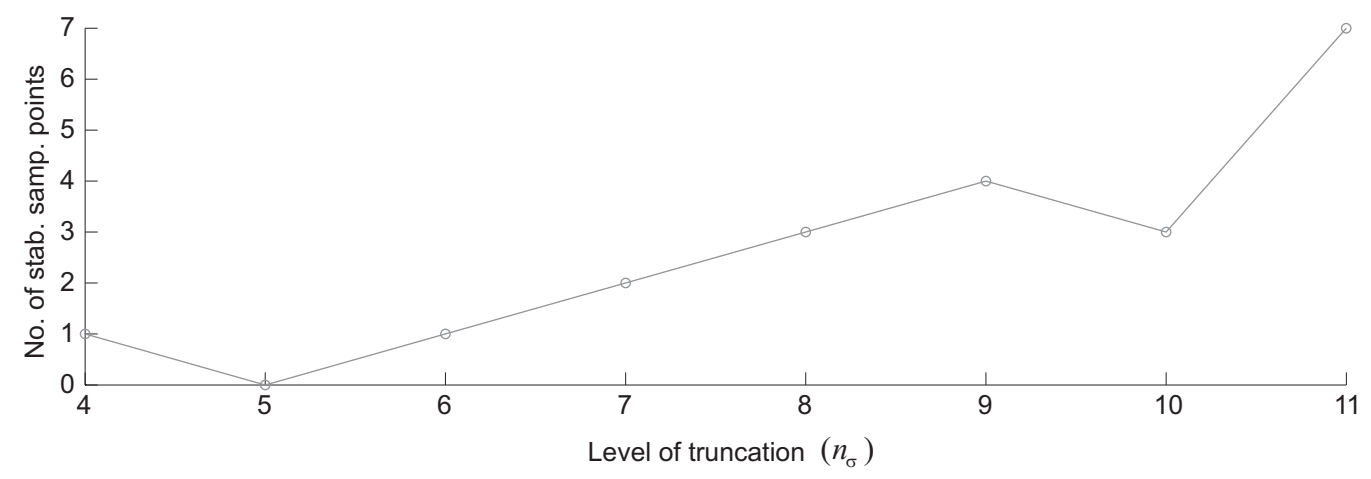

Figure 9: Minimum number of stabilizing sampling points required to make the Jacobian matrix $\boldsymbol{K}^{*}$ definite positive for each level of truncation $n_{\sigma}=n_{u}=p_{\sigma}$ (deduced from Figure 8).

the level of truncation (and thus the number of basic sampling points), the more stabilizing sampling points appear to be needed to ensure the positive definiteness of $\boldsymbol{K}^{*}$. For $n_{\sigma}=p_{\sigma}=6$, adding just one stabilizing sampling points suffices, while for $n_{\sigma}=p_{\sigma}=11,7$ points are required.

Observation 9.1. The values shown in Figure 9 correspond to the minimum $p_{B}$ that leads to positive definite $\boldsymbol{K}^{*}$ when the prescribed strain path coincides with any of the "training" strain trajectory (displayed in Figure 4 ). Unfortunately, there is no guarantee that the Jacobian matrix will also exhibit this desirable property for prescribed strain histories different from the training ones. Thus, in view of such uncertainty, and in the interest of robustness, it is preferable to stay on the side of "caution" in this regard and use more 


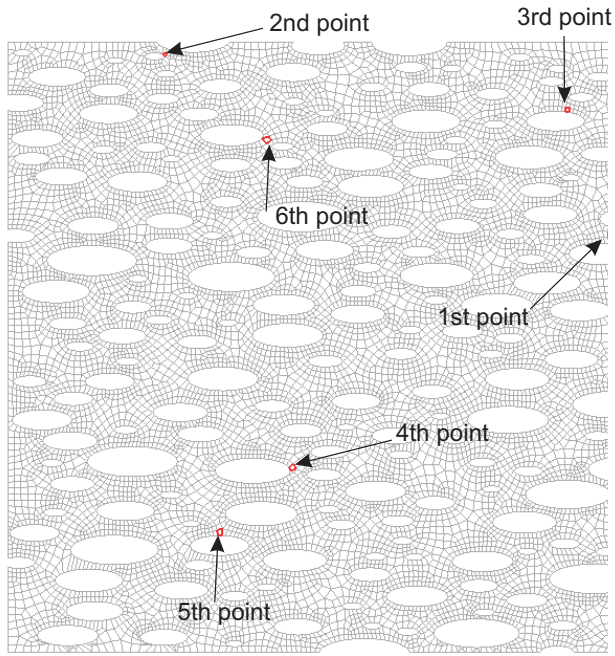

a) Basic sampling points

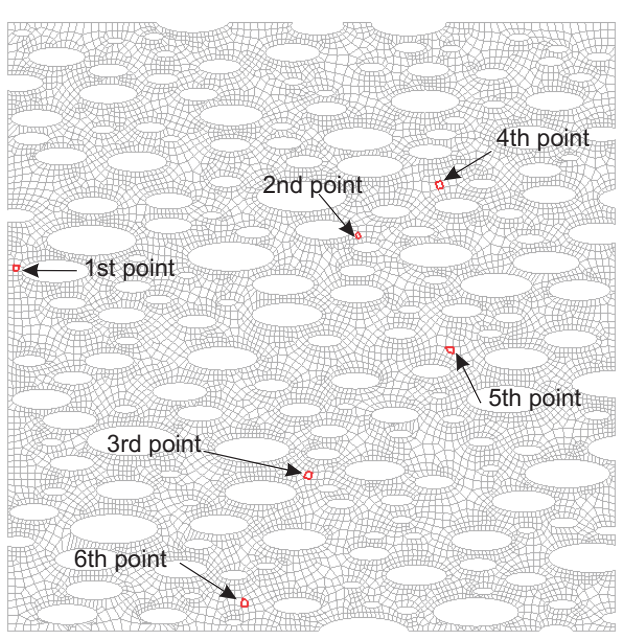

b) Stabilizing sampling points

Figure 10: Location within the RVE of the finite elements (marked in red) that contains the first $p_{\sigma}=p_{B}=6$ basic and stabilizing sampling points.

stabilizing sampling points that the minimum number indicated by the analysis based on the training strain trajectories. It is the authors' experience that a "safe" estimate for $p_{B}$ is to simply take $p_{B}=p_{\sigma}$-that is, equal number of basic and stabilizing sampling points. In adopting such a rule, the authors have not observed any convergence failures whatsoever, neither in the example under consideration nor in other cases not shown here.

The location of the first $p_{\sigma}=6$ basic sampling points and the corresponding $p_{B}=6$ stabilizing sampling points is depicted in Figure 10 .

\subsection{A posteriori errors: consistency analysis}

The error measures displayed previously in Figures 5 and 7 only depend on the outcome of the SVD of the snapshot matrices; they can be calculated, thus, before actually constructing the reduced-order model. Error analyses based on such measures serve the useful purpose of providing a first hint of how many stress and displacement fluctuations modes are needed to satisfactorily replicate the full-order, finite element solution, and thereby, of prospectively evaluating the viability of the reduced basis approach itself.

However, these a priori error estimates do not tell the whole story. Expression (121) for the stress approximation error presumes that the stress 
solution at the chosen sampling points is the one provided by the finite element model, thus ignoring the fact that, actually, in the reduced-order model, and for the general case of nonlinear, dissipative materials, the stress information at such points at a given time step is already polluted by truncation (in displacement fluctuations and stresses) and reconstruction (in stresses) errors originated in previous time steps. To quantify the extent to which this amalgam of accumulated errors affects the predictions furnished by the HP-ROM, it is necessary to perform a consistency analysis.

Generally speaking, a reduced basis approximation is said to be consistent if, in the limit of no truncation, it introduces no additional error in the solution of the same problem for which the data used in constructing the basis functions were acquired [14]. In the BVP under consideration, thus, consistency implies that, when using as input macro-strain paths the same trajectories employed in the "training" process, results obtained with the HP-ROM should converge, as $n_{\sigma}$ and $n_{u}$ increase, to the solution furnished by the full-order, finite element model. This condition can be checked by

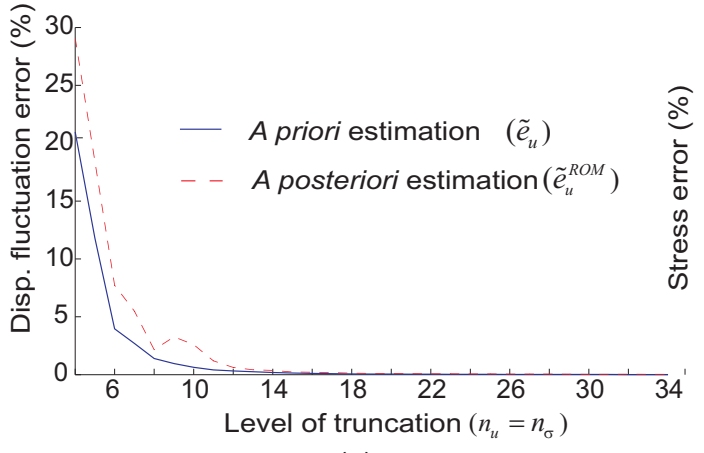

(a)

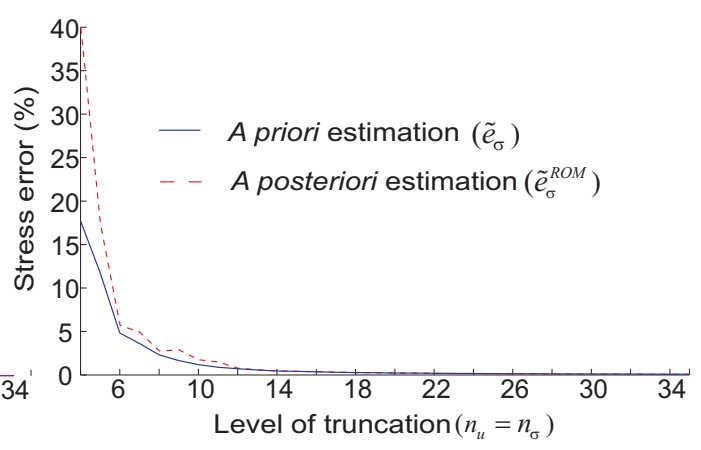

(b)

Figure 11: Comparison of the evolution of a priori and a posteriori error measures versus the level of truncation (using $n_{u}=n_{\sigma}=p_{\sigma}=p_{B}$ ). a) Displacement fluctuations (see Eqs. 119 and 123). b) Stresses (see Eqs. 121 and 124)

studying the evolution of the error measures defined as

$$
\tilde{e}_{u}^{R O M}\left(n_{u}, n_{\sigma}, \mathcal{I}\right):=\frac{\left\|\overline{\boldsymbol{X}}_{u}-\overline{\boldsymbol{X}}_{u}^{* R O M}\left(n_{u}, n_{\sigma}, \mathcal{I}\right)\right\|_{F}}{\left\|\overline{\boldsymbol{X}}_{u}\right\|_{F}},
$$

for the displacement fluctuations, and

$$
\tilde{e}_{\sigma}^{R O M}\left(n_{u}, n_{\sigma}, \mathcal{I}_{\sigma}\right):=\frac{\left\|\boldsymbol{X}-\boldsymbol{X}^{* R O M}\left(n_{u}, n_{\sigma}, \mathcal{I}_{\sigma}\right)\right\|_{F}}{\|\boldsymbol{X}\|_{F}},
$$




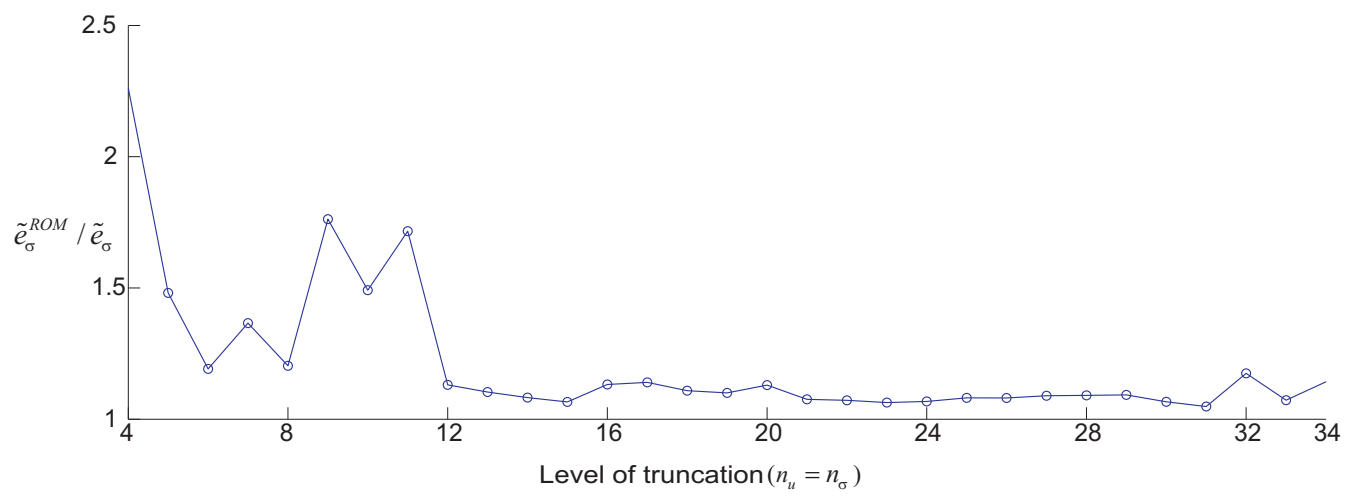

Figure 12: Ratio $\tilde{e}_{\sigma}^{R O M} / \tilde{e}_{\sigma}$ between the a posteriori and a priori measures for the stress approximation error against the level of truncation (using $n_{u}=n_{\sigma}=p_{\sigma}=p_{B}$ ).

for the stresses. ( The superscript "ROM" is appended to highlight that, unlike $\overline{\boldsymbol{X}}_{u}^{*}$ and $\boldsymbol{X}^{*}$ in Eqs. (119) and (121), $\overline{\boldsymbol{X}}_{u}^{* R O M}$ and $\boldsymbol{X}^{* R O M}$ are matrices of displacement fluctuation and stress snapshots computed using the HPROM). Figures 11.a and 11.b contain the graphs of these a posteriori error measures, along with their respective a priori counterparts $\tilde{e}_{u}$ (Eq. 119) and $\tilde{e}_{\sigma}$ (Eq. 121), versus the level of truncation. It becomes clear from these graphs that consistency, in the sense given above, is observed in terms of both displacement fluctuations and stresses: the a posteriori error measures $\tilde{e}_{u}^{R O M}$ and $\tilde{e}_{\sigma}^{R O M}$ mimic essentially the decreasing tendency of their a priori counterparts $\tilde{e}_{u}$ and $\tilde{e}_{\sigma}$, respectively. It can be seen also that the a priori error estimations $\tilde{e}_{u}$ and $\tilde{e}_{\sigma}$ constitute (rather tight) lower bounds for their a posteriori counterparts $\tilde{e}_{u}^{R O M}$ and $\tilde{e}_{\sigma}^{R O M}$, respectively. This can be better appreciated, for the stresses, in Figure 12, where the ratio $\tilde{e}_{\sigma}^{R O M} / \tilde{e}_{\sigma}$ versus the level of truncation is plotted.

The degree of approximation that can be achieved using the proposed HP-ROM is quantified in a more "engineering" fashion in Figure 13, where we plot, for the case of the first training strain history (stretching in the longitudinal direction), the longitudinal, macroscopic stress-strain curves computed using the FE model, on the one hand, and the HP-ROM with $n_{u}=n_{\sigma}=6,7,8$ modes, on the other hand. Observe that the maximum deviation from the $\mathrm{FE}$ response when using 6 modes (3 elastic and 3 inelastic) takes place at the onset of plastic yielding and is below $8 \%$; remarkably, as deformation continues, this deviation gradually diminishes, being practically 


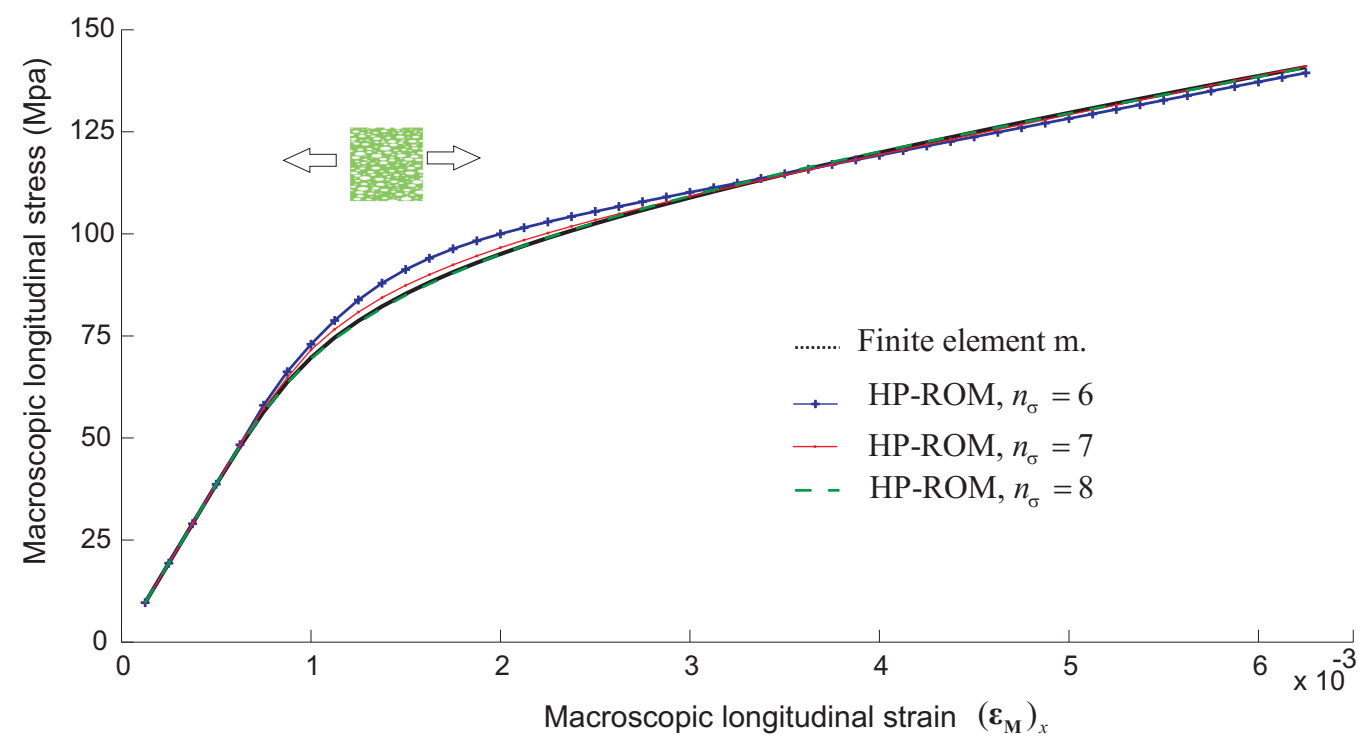

Figure 13: Longitudinal macroscopic stress versus longitudinal macroscopic strain computed using FEM and HP-ROM with $n_{\sigma}=n_{u}=6,7,8$.

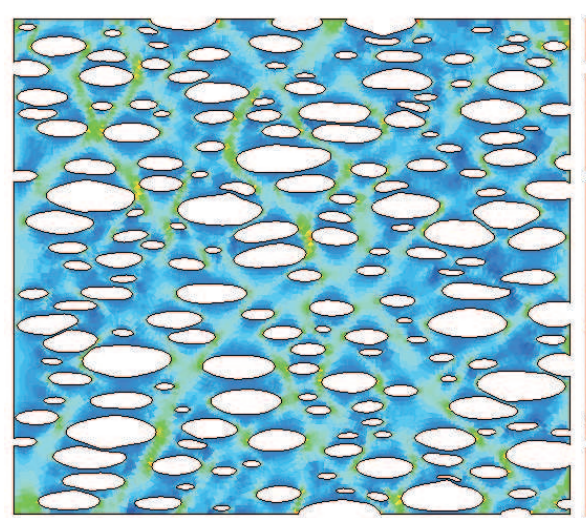

a) Finite element model

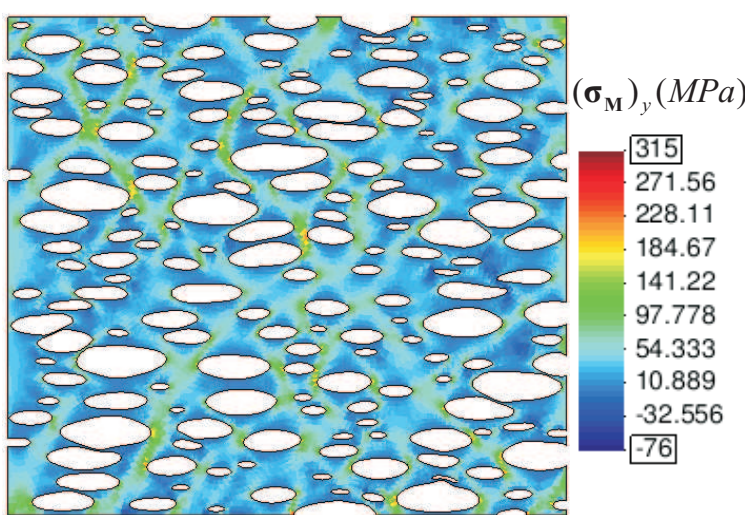

b) HP-ROM $\left(n_{\sigma}=n_{u}=6\right)$

Figure 14: Contour plot of transversal stresses computed at the end of the first "training" strain history using a) FEM (b) HP-ROM with $n_{\sigma}=n_{u}=6$. Deformed shapes are exaggerated (by a factor of 20).

negligible at the end of the process. Furthermore, by just increasing the order of truncation to $n_{\sigma}=n_{u}=8$, differences between the HP-ROM and the FEM responses become virtually imperceptible at all levels of deformation. 
Resemblance between HP-ROM and FEM results can also be appreciated in terms of stress distribution in the contour plots shown in Figure 14. Visually, there are no discernible differences between the two contour plots.

\section{7. "Training" errors}

The studies presented in the preceding subsections were aimed at examining the errors incurred in approximating the snapshot solution space $\mathcal{V}_{u}^{s n p}$ by the reduced-order subspace $\mathcal{V}_{u}^{*} \in \mathcal{V}_{u}^{s n p}$ spanned by the POD basis vectors -in the terminology of Section 3.1-, and to check that when $\mathcal{V}_{u}^{*} \rightarrow \mathcal{V}_{u}^{s n p}$, the solution provided by the HP-ROM converges to that obtained with the FEM. But recall that the snapshot space $\mathcal{V}_{u}^{s n p}$ is but a (presumably representative) subspace of $\mathcal{V}_{u}^{\epsilon}$, the manifold of $\mathcal{V}_{u}^{h}$ induced by the parametric dependence of the fine-scale BVP on the prescribed macroscopic strain history. Consequently, in general - for an arbitrary input strain trajectory - the HP-ROM solution will not converge to the solution provided by the FEM. To complete the error assessment analysis, thus, it is necessary to estimate also the errors inherent to the sampling of the parameter space —we call them training errors - and judge whether the selected training strain trajectories generate a snapshot subspace that is indeed representative of such a solution space ${ }^{21}$ $\mathcal{V}_{u}^{\epsilon}$.

Ideally, one should carry out this error assessment by picking up, guided by some sound, statistically-based procedure, a sufficiently large set of strain paths and by comparing the solutions computed by the FEM and HP-ROM under such input strain paths for varying levels of truncation. Such a degree of rigor, however, is beyond the scope of the present work. Here, we limit ourselves here to analyze the quality of the HP-ROM approximation obtained for two different input strain histories, namely, a uniaxial compression test, and a biaxial loading/unloading test.

\footnotetext{
${ }^{21}$ To put it in less mathematical terms - by appealing to the the analogy, introduced in Remark 9.1, between the training of the RVE reduced-order model and the calibration of standard phenomenological models - we have "calibrated" our HP-ROM using the training tests displayed previously in Figure 4, and we have shown that the model is able to exactly replicate the behavior of the RVE in these tests when $n_{u}=n_{\sigma}$ is sufficiently large. Similarly to the situation encountered when dealing with standard phenomenological models, it remains now to assess the capability of the proposed HP-ROM to predict the behavior of the RVE under conditions different from those used in the "calibration" (training) process.
} 


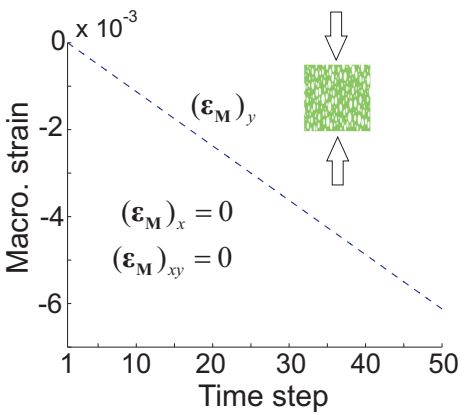

a)

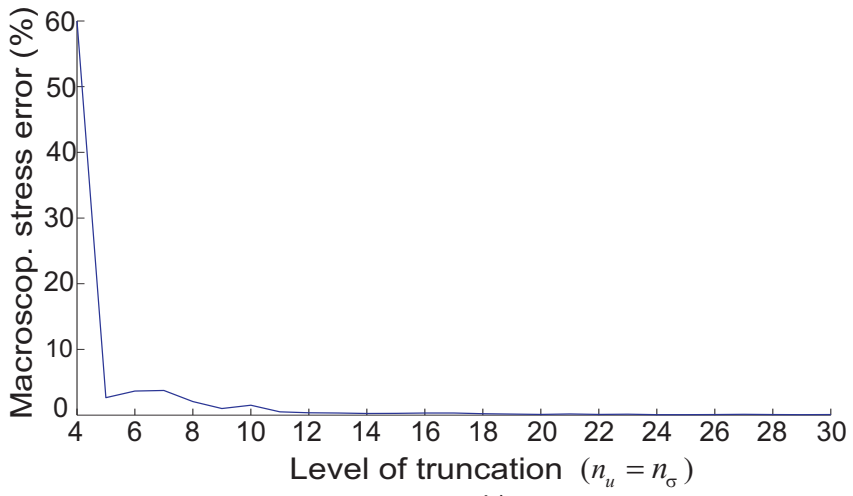

b)

Figure 15: a) First strain trajectory employed for assessing training errors. b) Plot of the macroscopic error estimator $\tilde{E}_{\sigma, M}^{R O M}$ (see Eq.(125)) corresponding to this testing trajectory versus level of truncation $\left(n_{\sigma}=n_{u}\right)$

\subsubsection{Uniaxial compression}

The first strain path employed for the assessment is displayed in Figure 15.a; it represents a monotonic compression in the transversal direction (the model, see Figure 4, was trained using only stretching and shear, but not compression, tests). For purposes of evaluating the quality of the HP-ROM approximation, it is convenient to introduce the following macroscopic ${ }^{22}$ stress error estimate:

$$
\tilde{E}_{\sigma, M}^{R O M}:=\sqrt{\frac{\sum_{i=1}^{n_{s t p}^{t}}\left\|\boldsymbol{\sigma}_{M}{ }^{i}-\boldsymbol{\sigma}_{M}^{* i, R O M}\left(n_{\sigma}, n_{u}, \mathcal{I}\right)\right\|^{2}}{\sum_{i=1}^{n_{s t p}^{t}}\left\|\boldsymbol{\sigma}_{M}\right\|^{i} \|^{2}}},
$$

where $\boldsymbol{\sigma}_{M}{ }^{i}$ and $\boldsymbol{\sigma}_{M}^{* i, R O M}$ denote the macroscopic stress at the $i-t h$ time step computed by the FEM and the HP-ROM, respectively. This error estimate is plotted in Figure 15.b versus the level of truncation $n_{u}=n_{\sigma}$. Observe that the error goes to zero as the number of employed modes increase. In this particular case, thus, there is no additional error due to sampling of the

\footnotetext{
${ }^{22}$ Recall that the output of interest in solving the fine-scale BVP is the macroscopic stress tensor; thus, the error estimate defined in Eq.(125) $\left(\tilde{E}_{\sigma, M}^{R O M}\right)$ provides a more meaningful indication of the quality of the approximation than the stress error measure defined previously in Eq.(124) $\left(\tilde{e}_{\sigma}^{R O M}\right)$. The latter is more suited for examining convergence properties of the HP-ROM approximation, since the minimization problem that underlies the SVD is posed in terms of the Frobenis norm.
} 
parameter space.

Remark 9.2. This simple example fittingly illustrates one of the acclaimed advantages of POD/Galerkin reduced-order approaches over "black box" methods such as artificial neural networks - that are also based on the partitioned offline-online computational paradigm-: POD/Galerkin reduced-order approaches preserve the "physics" of the problem one wishes to model and, as a consequence, are able to make physically-based extrapolations. For instance, in this case, the reduced-order model is able to exactly replicate (for sufficiently large $n_{u}=n_{\sigma}$ ) the macroscopic compressive behavior of the RVE, even though no information regarding this deformational state has been supplied to the model in the calibration (training) phase; the HP-ROM is "aware", figuratively speaking, that the matrix material in the RVE exhibits similar behavior in tension and compression (J2 plasticity).

\subsubsection{Biaxil loading/unloading test}

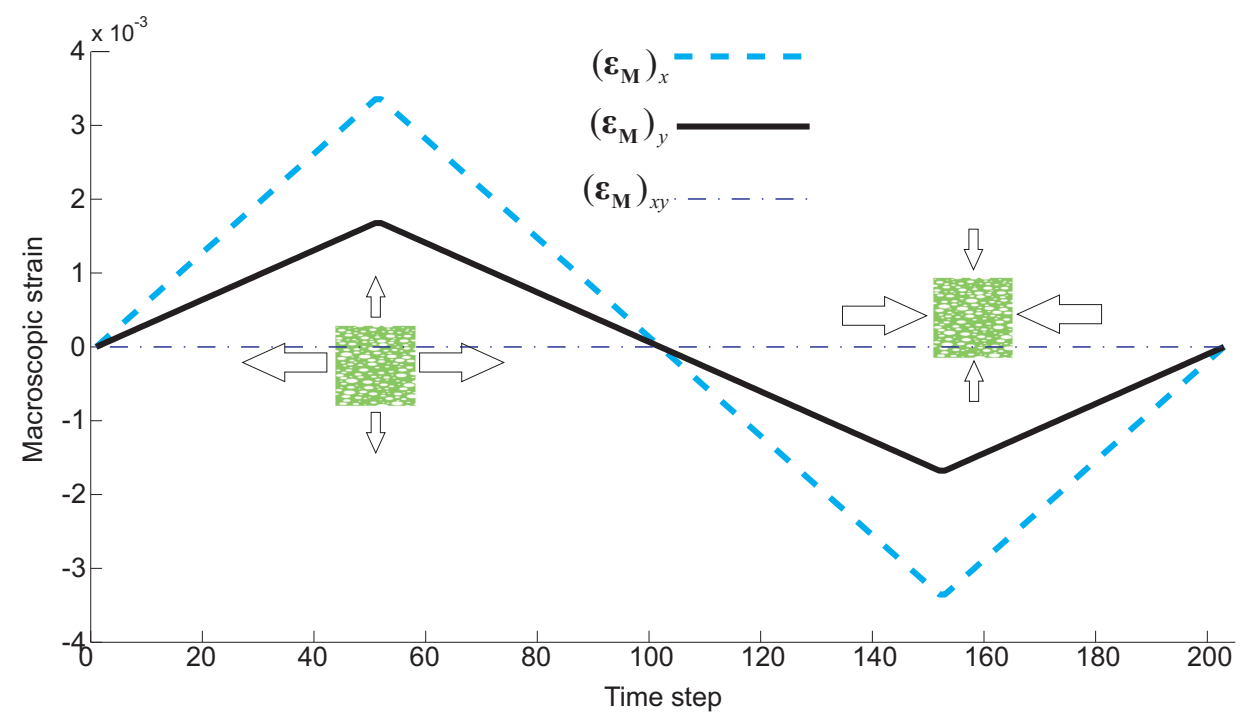

Figure 16: Second strain trajectory employed for assessing training errors.

A more severe test for assessing errors associated to the training process is provided by the strain trajectory shown in Figure 16. Indeed, while the training strain histories of Figure 4 only included monotonic, uniaxial stretching, the strain history displayed in Figure 16 consists of a cycle of biaxial, loading/unloading stretching (time steps 1 to 100) and biaxial loading/unloading 

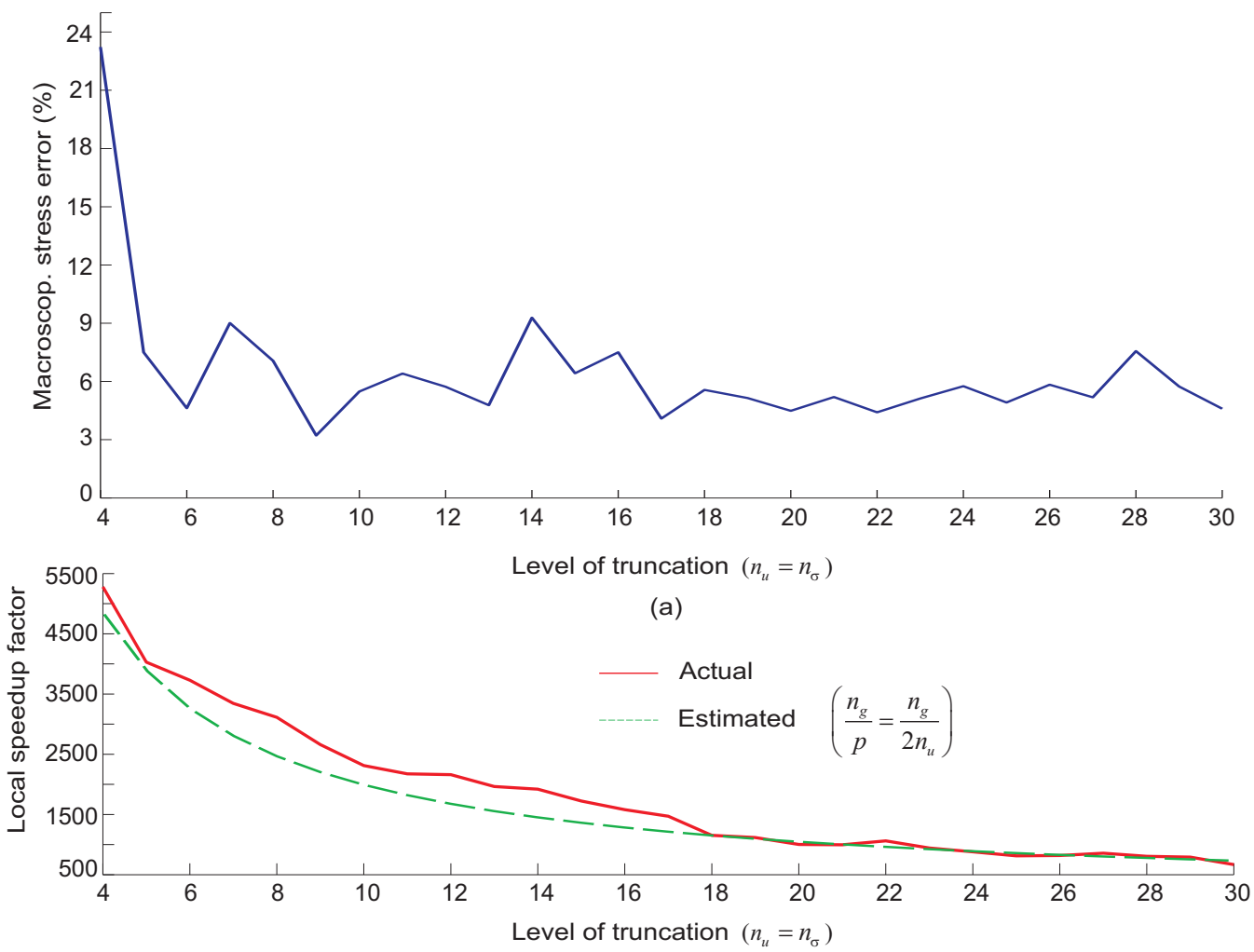

(b)

Figure 17: a) Macroscopic error estimator $\tilde{E}_{\sigma, M}^{R O M}$ (see Eq.(125)) versus level of truncation $\left(n_{\sigma}=n_{u}\right)$ for the case of testing trajectory shown in Figure 16,. b) Local speedup factor $S_{l o c}$ (defined in Eq.(126)) reported for this case versus level of truncation. This plot is accompanied by the graph of the ratio $n_{g} / p$, where $n_{g}=38984$ is the total number of Gauss points of the finite element mesh, and $p=2 n_{\sigma}$ the number of sampling points employed for numerically integrating the HP-ROM.

compression (time steps 101 to 200). The graph of the macroscopic error estimator (125) corresponding to this input strain path as a function of the level of truncation is represented in Figure 17.a. It can be readily perceived that, in this case, and in contrast to the situation encountered in the previously discussed input strain trajectory, the macroscopic stress does not go to zero as the number of POD modes included in the basis increases. Rather, the graph drops sharply from $24 \%$ to approximately $5 \%$ at $n_{\sigma}=n_{u}=5$ (second inelastic mode), and then fluctuates erratically, with no apparent trend, between $3 \%$ and $10 \%$ - a level of accuracy that, nevertheless, may be deemed 


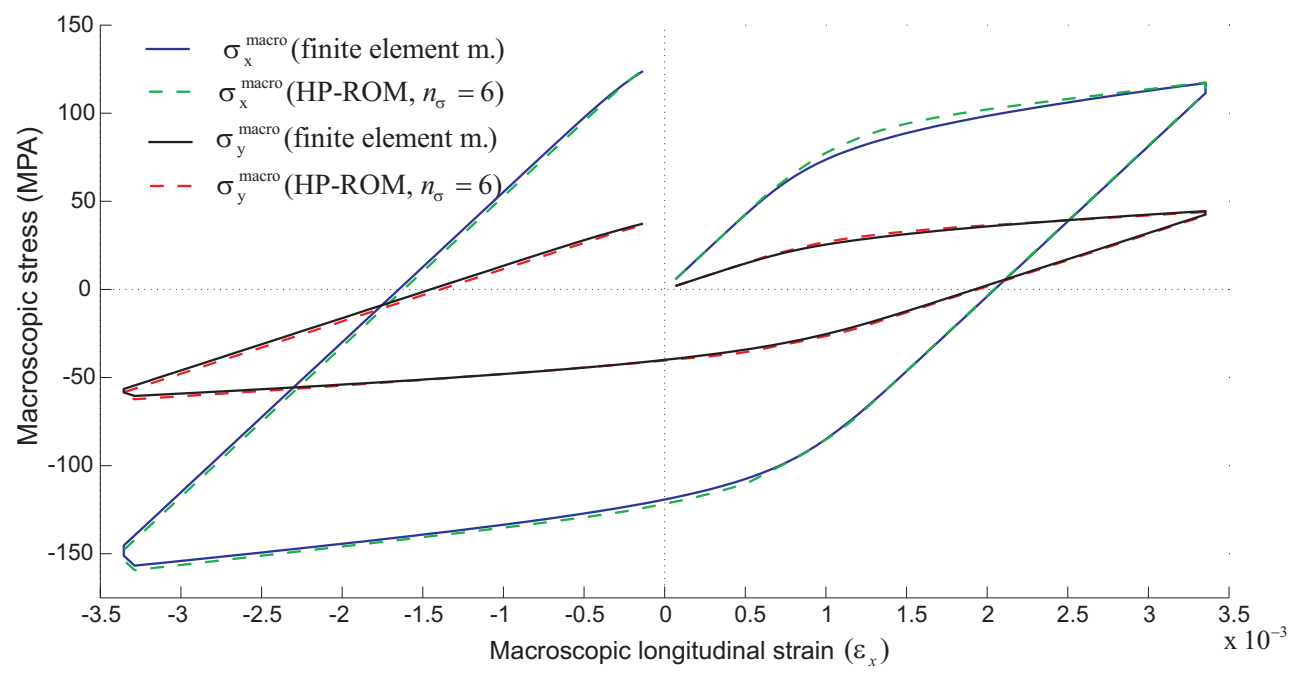

Figure 18: Longitudinal and transversal macroscopic stress versus longitudinal macroscopic strain computed using the FEM and the HP-ROM with $n_{\sigma}=n_{u}=6$ (for the case of the testing trajectory shown in Figure 16)

more than acceptable in most practical applications. A more clear picture of the accuracy of the approximation for the particular case of $n_{\sigma}=n_{u}=6$ can be obtained from the stress-strain diagrams shown in figure 18 .

\subsection{Speedup analysis}

Lastly, we turn our attention to one of the main concerns of the present work: the issue of computational efficiency. For a given error level, how many times can the proposed HP-ROM speed up the calculation of the RVE response with respect to the reference finite element model? Let us define the local speedup factor as the ratio

$$
S_{l o c}:=\frac{t_{F E}\left(n, n_{g}\right)}{t_{R O M}\left(n_{\sigma}, n_{u}, p\right)},
$$

where $t_{F E}$ and $t_{R O M}$ denote the $\mathrm{CPU}$ times required to compute the $\mathrm{FE}$ and HP-ROM macro-stress responses, respectively, induced by a given input strain history ${ }^{23}$ In Figure 17.b, we show the graph of the speedup factor

\footnotetext{
${ }^{23}$ The computational cost associated to the offline stage - generation of snapshots plus the comparatively negligible expenses of applying the POD and selecting the sampling
} 
reported in the the case of the input strain path of Figure 16 as a function of the number of POD modes included in the analysis (recall in this respect that $n_{u}=n_{\sigma}=p / 2$ ). We plot also in Figure 17.b the ratio $n_{g} / p$, i.e., the relation between the total number of integration points in the finite element model $\left(n_{g}=38984\right)$ and in the reduced order model $(p)$. It can be gleaned from Figure 17.b that the reported speedup factors are of the same order of magnitude as the ratio $n_{g} / p$; i.e.:

$$
S_{l o c} \sim \frac{n_{g}}{p}=\frac{n_{g}}{2 n_{\sigma}}
$$

(this indicates that the evaluation of the stresses at the integration points dominates the total computational cost). Although these results are no doubt influenced and biased by the particular programming language and coding style employed — we use an in-house, non-vectorized Matlab program operating in a Linux platform-, and, consequently, this trend may not be exactly observed when using other programming languages and/or platforms, they serve to provide an idea of the tremendous gains in performance that can be achieved using the proposed ROM; for $n_{\sigma}=p=6$ modes, for instance, the computational cost is reduced by a factor above 3600, while still capturing $95 \%$ of the full-order, high-fidelity information - the essential information.

\section{Concluding remarks}

One of the the most striking features of the proposed reduced-order model is perhaps the conceptual simplicity of the RVE equilibrium equation in its hyperreduced-order form: the sum of (reduced) internal forces at the preselected sampling points must give identical result either calculated using observed stresses or fitted stresses. Although this condition appears, in hindsight, rather reasonable, even obvious - it ensures maximum resemblance between reduced-order and full-order responses at the sampling points - it seems difficult to arrive at it without the benefit of the integration procedure - based on the notion of expanded approximation space - advocated in the present paper.

points - has been deliberately ruled out from this speedup analysis because, in two-scale homogenization contexts, the RVE equilibrium problem is to be solved a sheer number of times and, consequently, this overhead cost is quickly amortized. 
The hyperreduced form of the RVE equilibrium equation excels not only in its conceptual simplicity; the corresponding solution scheme is also very simple to implement. Taking as departure point an existing FE code, one only has to replace the typical loop over elements in the FE code by a loop over the pre-selected sampling points $\left\{\mathbf{x}_{\mathcal{I}_{1}}, \mathbf{x}_{\mathcal{I}_{2}}, \ldots \mathbf{x}_{\mathcal{I}_{p}}\right\}$. The stress vectors and corresponding constitutive tangent matrices obtained at each stage of the loop are stored in the gappy weighted vector $\hat{\Sigma}$ and the matrix $\hat{\mathbb{C}}$, respectively, and, then the residual vector and the Jacobian matrix are computed as $\hat{\mathbb{B}}^{* *^{T}} \hat{\Sigma}$ and $\hat{\mathbb{B}}^{* *} \hat{\mathbb{C}} \hat{\mathbb{B}}^{*}$, respectively. Notice that no assembly process is needed, nor has one to worry about imposing boundary conditions. Once convergence is achieved, the macroscopic stress value is simply calculated as $\boldsymbol{\sigma}_{\boldsymbol{M}}=\mathbb{T} \hat{\boldsymbol{\Sigma}}$. It should be emphasized again that the operation count in both solving this hyperreduced RVE equation and updating the macroscopic stress vector depends exclusively on the reduced dimensions $n_{u}$ and $p$ (number of fluctuation modes and number of sampling points, respectively). Likewise, storage of history data (internal variables) is only required at the pre-selected sampling points. Computational savings accrue, thus, not only in terms of number of operations, but also in terms of memory requirements.

The success of the proposed homogenization strategy is predicated on the assumption that displacement and stress fields induced by the parametric dependence on the input macroscopic strain can be approximated by (relatively) low-dimensional functions. Numerical results shown in the preceding section seem to suggest that, in general, this assumption may be expected to hold in the case of materials governed by strain hardening laws in the small strain regime - regardless of the geometrical complexity, number and distribution of heterogeneities within the RVE. However, it is by no means apparent that this conclusion can be easily extended to more (kinematically and phenomenologically) complex scenarios, involving large deformations, strain localization, decohesion, etc. For instance, can the deformational behavior of an RVE affected by multiple propagating cracks be represented also in a parsimonious manner, as in the case of strain hardening? Or will the number of modes necessary to accurately replicate its response combinatorially increase with the number of potential crack paths (i.e., with the geometrical complexity of the RVE)? Undoubtedly, in these complex, nonlinear scenarios, the task of sampling the parameter space -i.e., of choosing the macro-strain histories at which to obtain the snapshots - will become quite complicated, due to the richness of possible deformational patterns (void closure, propagating discontinuities ...), and thus difficult to carry out 
on intuitive basis (as it has been done in the present paper). Therefore, it would be desirable to systematize this crucial task, as well as to provide some statistical means to certify, so to speak, the representativeness of the chosen snapshots. Likewise, topological variations caused by these phenomena may render POD-based compression algorithms inappropriate for collapsing the dimensions of the snapshots space; in such cases, nonlinear dimensionality reduction methods such as the Isomap algorithm [43] may be more suitable. Research in these fronts is currently in progress and will be reported in forthcoming publications.

\section{Acknowledgements}

The research leading to these results has received funding from, on the one hand, the European Research Council under the European Union's Seventh Framework Programme (FP/2007-2013) / ERC Grant Agreement n. 320815, Advanced Grant Project COMP-DES-MAT, and, on the other hand, the Spanish Ministry of Science and Innovation under grant BIA2011-24258.

\section{A. Proper Orthogonal Decomposition}

The formal statement of the POD problem goes as follows: given the ensemble of snapshots $\left\{\boldsymbol{u}^{1}, \boldsymbol{u}^{2}, \ldots \boldsymbol{u}^{n_{s n p}}\right\}$, find a set of $n_{u}<n_{s n p}$ orthogonal basis functions $\left\{\boldsymbol{\Phi}_{1}, \boldsymbol{\Phi}_{2}, \ldots \boldsymbol{\Phi}_{n_{u}}\right\}\left(\boldsymbol{\Phi}_{i} \in \mathcal{V}_{u}^{s n p}\right)$ such that the error defined as

$$
e_{u}\left(n_{u}\right):=\sqrt{\sum_{k=1}^{n_{s n p}}\left\|\boldsymbol{u}^{k}-\mathbf{P}^{*} \boldsymbol{u}^{k}\right\|_{L_{2}(\Omega)}^{2}}
$$

is minimized. Here, $\mathbf{P}^{*} \boldsymbol{u}^{k}$ represents the projection of $\boldsymbol{u}^{k}$ onto the subspace spanned by the basis functions $\left\{\boldsymbol{\Phi}_{i}\right\}_{i=1}^{n_{u}}$, and $\|\cdot\|_{L_{2}(\Omega)}$ symbolizes the $L_{2}$ norm. We shall denote by $\boldsymbol{\Phi}_{i}\left(i=1,2 \ldots n_{u}\right)$ the column vector containing the values of basis function $\boldsymbol{\Phi}_{i}$ at the nodes of the underlying finite element mesh. Likewise, the matrix formed by such vectors, $\boldsymbol{\Phi}=\left[\begin{array}{lll}\boldsymbol{\Phi}_{1} & \boldsymbol{\Phi}_{2} & \cdots\end{array}\right] \in \mathbb{R}^{n \cdot d \times n_{u}}$, will be hereafter called the reduced basis matrix.

The reduced basis matrix $\boldsymbol{\Phi}$ can be computed from the snapshot matrix $\boldsymbol{X}_{u}$ by means of the Singular Value Decomposition (SVD) as follows (see 
Ref. [16] for more details). We first define the matrix ${ }^{24}$

$$
\mathbf{M}_{I J}:=\int_{\Omega} N_{I}(\mathbf{x}) N_{J}(\mathbf{x}) d \Omega \quad I, J=1,2 \ldots n .
$$

Let $\mathbf{M}=\overline{\mathbf{M}}^{T} \overline{\mathbf{M}}$ be the Cholesky decomposition of $\mathbf{M}$, and let $\overline{\boldsymbol{X}}_{u}$ denote the matrix defined as:

$$
\overline{\boldsymbol{X}}_{u}:=\overline{\mathbf{M}} \boldsymbol{X}_{u}
$$

Then, we compute the reduced SVD [37] of $\overline{\boldsymbol{X}}_{u}$, that is, the factorization

$$
\overline{\boldsymbol{X}}_{u}=\overline{\boldsymbol{U}} \overline{\boldsymbol{S}} \overline{\boldsymbol{V}}^{T}
$$

where $\overline{\boldsymbol{V}} \in \mathbb{R}^{n_{s n p} \times r}\left(r\right.$ is the rank of $\left.\overline{\boldsymbol{X}}_{u}\right)$ and $\overline{\boldsymbol{U}} \in \mathbb{R}^{n \cdot d \times r}$ stand for the matrices of right and left singular vectors, respectively; and $\overline{\boldsymbol{S}} \in \mathbb{R}^{r \times r}$ is a diagonal matrix containing the singular values of $\boldsymbol{X}_{u}$. The $i-t$ column of the reduced basis matrix $\boldsymbol{\Phi}$ is finally related to the $i-t h$ left singular vector of $\overline{\boldsymbol{X}}_{u}$ through expression

$$
\boldsymbol{\Phi}_{i}=\overline{\mathbf{M}}^{-1} \overline{\boldsymbol{U}}_{i}, \quad i=1,2 \ldots n_{u} .
$$

\section{B. Elastic/Inelastic reduced basis matrix}

This appendix is devoted to provide further details concerning the actual numerical implementation of the elastic/inelastic partitioned strategy, presented in Section 3.2.1, for the computation of the reduced basis matrices $\mathbf{\Phi}$ (displacement fluctuations). The steps to arrive at the desired matrix basis $\boldsymbol{\Phi}$ are summarized in the following.

1. Compute finite element stress solutions for representative, input macrostrain histories.

2. Store the displacement fluctuation solutions computed at each time step of these macro-strain trajectories in the displacement fluctuations snapshot matrix $\boldsymbol{X}_{u} \in \mathbb{R}^{n \cdot d \times n_{s n p}}$ :

$$
\boldsymbol{X}_{u}=\left[\begin{array}{llll}
\boldsymbol{U}^{1} & \boldsymbol{U}^{2} & \cdots & \boldsymbol{U}^{n_{s n p}}
\end{array}\right]
$$

\footnotetext{
${ }^{24}$ Note that, except for the density factor, this matrix $\mathbf{M}$ is similar to the "mass matrix" appearing in finite element implementations of dynamical problems. For implementational purposes, one can simply use a lumped version of such a matrix.
} 
3. Pick up from $\boldsymbol{X}_{u}$ a minimum of $m_{e}\left(m_{e}=6\right.$ for $3 \mathrm{D}$ problems, and $m_{e}=3$ for plane strain) linearly independent columns corresponding to purely elastic solutions. Store these columns in a matrix $\boldsymbol{Z}_{u}^{e l}$.

4. Perform the reduced singular value decomposition (SVD) of the matrix defined as

$$
\overline{\boldsymbol{Z}}_{u}^{e l}:=\overline{\mathbf{M}} \boldsymbol{Z}_{u}^{e l}
$$

where $\overline{\mathbf{M}}$ is the matrix of the Cholesky factorization of $\mathbf{M}$ ( $\mathbf{M}=$ $\left.\overline{\mathbf{M}}^{T} \overline{\mathbf{M}}\right)$. A basis matrix for $\operatorname{Range}\left(\boldsymbol{Z}_{u}^{e l}\right)$ is finally obtained as

$$
D^{e l}:=\overline{\mathbf{M}}^{-1} \bar{D}^{e l}
$$

$\overline{\boldsymbol{D}}^{e l} \in \mathbb{R}^{n \cdot d \times m_{e}}$ being the matrix of left singular vectors arising from the SVD of $\overline{\boldsymbol{Z}}_{u}^{e l}$. In principle, $\boldsymbol{D}^{e l}$ may be used as the desired elastic basis matrix $\boldsymbol{\Phi}^{e l}$. However, $\boldsymbol{D}^{e l}$ does not enjoy any optimality property with respect to $\boldsymbol{X}_{u}$-it is only optimal with respect to the matrix $\boldsymbol{Z}_{u}^{e l}$ of chosen elastic snapshots.

5. For consistency in the approximation, thus, it is preferable to derive $\boldsymbol{\Phi}^{e l}$ from the the "elastic component" of $\boldsymbol{X}_{u}$-the orthogonal projection of $\boldsymbol{X}_{u}$ onto Range $\left(\boldsymbol{D}^{e l}\right)$-; the expression for this projection reads:

$$
\boldsymbol{X}_{u}^{e l}=\boldsymbol{D}^{e l}\left(\boldsymbol{D}^{e l} \mathbf{M} \boldsymbol{X}_{u}\right)
$$

The elastic basis matrix can be finally calculated from $\boldsymbol{X}_{u}^{e l}$ as:

$$
\Phi^{e l}:=\overline{\mathbf{M}}^{-1} \overline{\boldsymbol{\Phi}}^{e l} \text {. }
$$

where $\overline{\boldsymbol{\Phi}}^{e l}$ is the matrix of left singular vectors emerging from the reduced SVD of $\overline{\boldsymbol{X}}_{u}^{e l}:=\overline{\mathbf{M}} \boldsymbol{X}_{u}^{e l}$; i.e $\overline{\boldsymbol{X}}_{u}^{e l}=\overline{\boldsymbol{\Phi}}^{e l} \overline{\boldsymbol{S}}_{u}^{e l} \overline{\boldsymbol{V}}_{u}^{e l^{T}}$.

6. Calculate the "inelastic component" $\boldsymbol{X}_{u}^{i n}$ of the snapshot matrix $\boldsymbol{X}_{u}$ as:

$$
\boldsymbol{X}_{u}^{i n}=\boldsymbol{X}_{u}-\boldsymbol{X}_{u}^{e l}
$$

that is, $\boldsymbol{X}_{u}^{i n}$ is the orthogonal projection of $\boldsymbol{X}_{u}$ onto the orthogonal complement, in Range $\left(\boldsymbol{X}_{u}\right)$, of Range $\left(\boldsymbol{\Phi}^{e l}\right)$.

7. It is now on this inelastic snapshot matrix $\boldsymbol{X}_{u}^{i n}$ that we apply the POD in order to identify and unveil the essential or most "energetic" inelastic fluctuation modes. This is done by first carrying out the reduced SVD of $\overline{\boldsymbol{X}}_{u}^{i n}=\overline{\mathbf{M}} \boldsymbol{X}_{u}^{i n}$ :

$$
\overline{\boldsymbol{X}}_{u}^{i n}=\overline{\boldsymbol{D}}^{i n} \overline{\boldsymbol{S}}_{u}^{i n} \overline{\boldsymbol{V}}_{u}^{i n^{T}}
$$


The $i-t h$ POD basis vector of $\boldsymbol{X}_{u}^{i n}$ is then given by:

$$
\boldsymbol{\Phi}_{i}^{i n}=\overline{\mathbf{M}}^{-1} \overline{\boldsymbol{D}}_{i}^{i n}, \quad i=1,2 \ldots n_{u}-m_{e} .
$$

8. The desired basis matrix $\boldsymbol{\Phi} \in \mathbb{R}^{n \cdot d \times n_{u}}$ adopts finally the form:

$$
\boldsymbol{\Phi}=\left[\boldsymbol{\Phi}^{e l} \boldsymbol{\Phi}^{i n}\right]=[\overbrace{\boldsymbol{\Phi}_{1}^{e l} \boldsymbol{\Phi}_{2}^{e l} \ldots \boldsymbol{\Phi}_{m_{e}}^{e l}}^{\text {Elastic modes }} \overbrace{\boldsymbol{\Phi}_{1}^{i n} \boldsymbol{\Phi}_{2}^{i n} \ldots \boldsymbol{\Phi}_{n_{u}-m_{e}}^{\text {in }}}^{\text {Essential inelastic modes }}]
$$

\section{Block matrix pseudoinverse of the expanded basis matrix}

The inverse of a $2 \times 2$ symmetric block matrix is given by the following expression (see, for instance, Ref. [11]):

$$
\mathbf{M}^{-1}=\left[\begin{array}{cc}
\mathrm{A} & \mathbf{B} \\
\mathrm{B}^{\mathrm{T}} & \mathrm{C}
\end{array}\right]^{-1}=\left[\begin{array}{cc}
\mathbf{A}^{-1}+\mathbf{A}^{-1} \mathbf{B S}^{-1} \mathbf{B}^{\mathrm{T}} \mathbf{A}^{-1} & -\mathbf{A}^{-1} \mathbf{B S}^{-1} \\
-\mathbf{S}^{-1} \mathbf{B}^{\mathrm{T}} \mathbf{A}^{-1} & \mathrm{~S}^{-1}
\end{array}\right]
$$

where

$$
\mathbf{S}=\mathbf{C}-\mathbf{B}^{\mathbf{T}} \mathbf{A}^{-1} \mathbf{B}
$$

is the so-called Schur complement of $\mathbf{A}$ in $\mathbf{M}$. This formula can be used to derive closed-form expressions for the modal coefficients $\boldsymbol{c}^{a d}$ and $\boldsymbol{c}^{\text {in }}$ (see Section 6.2). The departure point is equation Eq.(67):

$$
\left[\begin{array}{c}
\boldsymbol{c}^{a d} \\
\boldsymbol{c}^{i n}
\end{array}\right]=\left(\left[\hat{\boldsymbol{\Psi}} \hat{\mathbb{B}}^{*}\right]\right)^{\dagger} \hat{\boldsymbol{\Sigma}}=\left[\begin{array}{cc}
\hat{\mathbf{\Psi}}^{T} \hat{\mathbf{\Psi}} & \hat{\mathbf{\Psi}}^{T} \hat{\mathbb{B}}^{*} \\
\hat{\mathbb{B}}^{*^{T}} \hat{\boldsymbol{\Psi}} & \hat{\mathbb{B}}^{*^{T}} \hat{\mathbb{B}}^{*}
\end{array}\right]^{-1}\left[\begin{array}{c}
\hat{\mathbf{\Psi}}^{T} \\
\hat{\mathbb{B}}^{*^{T}}
\end{array}\right] \hat{\boldsymbol{\Sigma}},
$$

where $\left(\left[\hat{\mathbf{\Psi}} \hat{\mathbb{B}}^{*}\right]\right)^{\dagger}$ designates the pseudo-inverse of the gappy expanded basis matrix. By setting:

$$
\mathbf{A}=\hat{\mathbf{\Psi}}^{\mathbf{T}} \hat{\mathbf{\Psi}}, \quad \mathbf{B}=\hat{\mathbf{\Psi}}^{\mathbf{T}} \hat{\mathbb{B}}^{*}, \quad \mathbf{C}=\hat{\mathbb{B}}^{*^{\mathbf{T}}} \hat{\mathbb{B}}^{*},
$$

and by inserting Eq.(142) into Eq.(144), one obtains upon expansion:

$$
c^{i n}=\mathbf{S}^{-1}\left(-\mathbf{B}^{\mathbf{T}} \mathbf{A}^{-1} \hat{\Psi}^{\mathbf{T}}+\hat{\mathbb{B}}^{*^{\mathbf{T}}}\right) \hat{\boldsymbol{\Sigma}}
$$

and

$$
\begin{aligned}
c^{a d}= & \left(\left(\mathbf{A}^{-1}+\mathbf{A}^{-1} \mathbf{B} \mathbf{S}^{-1} \mathbf{B}^{\mathbf{T}} \mathbf{A}^{-1}\right) \hat{\Psi}^{T}-\mathbf{A}^{-1} \mathbf{B S}^{-1} \hat{\mathbb{B}}^{*^{\mathbf{T}}}\right) \hat{\Sigma} \\
= & \mathbf{A}^{-1} \hat{\Psi}^{\mathbf{T}} \hat{\Sigma}+\mathbf{A}^{-1} \mathbf{B} \overbrace{\mathbf{S}^{-1}\left(-\mathbf{B}^{\mathbf{T}} \mathbf{A}^{-1} \hat{\Psi}^{\mathbf{T}}+\hat{\mathbb{B}}^{*^{T}}\right) \hat{\Sigma}}^{c^{\text {in }}} \\
= & \mathbf{A}^{-1} \hat{\Psi}^{\mathbf{T}} \hat{\Sigma}+\mathbf{A}^{-1} \mathbf{B} c^{\text {in }} .
\end{aligned}
$$


By substituting back Eq.(145) into the above equation, and taking into account that:

$$
\hat{\boldsymbol{\Psi}}^{\dagger}=\left(\hat{\boldsymbol{\Psi}}^{T} \hat{\mathbf{\Psi}}\right)^{-1} \hat{\boldsymbol{\Psi}}^{T}
$$

one finally gets:

$$
\begin{gathered}
\boldsymbol{c}^{a d}=\hat{\Psi}^{\dagger}\left(\hat{\Sigma}-\hat{\mathbb{B}}^{*} c^{i n}\right), \\
c^{i n}=\mathbf{S}^{-\mathbf{1}} \hat{\mathbb{B}}^{*^{\mathrm{T}}}\left(\boldsymbol{I}-\hat{\Psi} \hat{\Psi}^{\dagger}\right) \hat{\boldsymbol{\Sigma}},
\end{gathered}
$$

where

$$
\mathbf{S}=\hat{\mathbb{B}}^{* \mathrm{~T}}\left(\boldsymbol{I}-\hat{\boldsymbol{\Psi}} \hat{\boldsymbol{\Psi}}^{\dagger}\right) \hat{\mathbb{B}}^{*}
$$

\section{References}

[1] Abdulle, A., Bai, Y., 2012. Adaptive reduced basis finite element heterogeneous multiscale method.

[2] Abdulle, A., Bai, Y., 2012. Reduced basis finite element heterogeneous multiscale method for high-order discretizations of elliptic homogenization problems. Journal of Computational Physics.

[3] An, S., Kim, T., James, D., 2009. Optimizing cubature for efficient integration of subspace deformations. ACM transactions on graphics $27(5), 165$.

[4] Ashby, M., 1992. Physical modelling of materials problems. Materials Science and Technology 8 (2), 102-111.

[5] Astrid, P., 2004. Reduction of process simulation models: a proper orthogonal decomposition approach. Technische Universiteit Eindhoven.

[6] Astrid, P., Weiland, S., Willcox, K., Backx, T., 2008. Missing point estimation in models described by proper orthogonal decomposition. Automatic Control, IEEE Transactions on 53 (10), 2237-2251.

[7] Barrault, M., Maday, Y., Nguyen, N., Patera, A., 2004. An empirical interpolation'method: application to efficient reduced-basis discretization of partial differential equations. Comptes Rendus Mathematique 339 (9), 667-672. 
[8] Bishop, C., en ligne), S. S., 2006. Pattern recognition and machine learning. Vol. 4. springer New York.

[9] Bohm, H., 1998. A short introduction to basic aspects of continuum micromechanics. CDL-FMD Report 3.

[10] Boyaval, S., 2007. Reduced-basis approach for homogenization beyond the periodic setting. Arxiv preprint math/0702674.

[11] Boyd, S., Vandenberghe, L., 2004. Convex optimization. Cambridge Univ Pr.

[12] Bui-Thanh, T., 2007. Model-constrained optimization methods for reduction of parameterized large-scale systems. Ph.D. thesis, Citeseer.

[13] Bui-Thanh, T., Willcox, K., Ghattas, O., 2008. Model reduction for large-scale systems with high-dimensional parametric input space. SIAM Journal on Scientific Computing 30 (6), 3270-3288.

[14] Carlberg, K., Bou-Mosleh, C., Farhat, C., 2011. Efficient non-linear model reduction via a least-squares petrov-galerkin projection and compressive tensor approximations. International Journal for Numerical Methods in Engineering 86 (2), 155-181.

[15] Carlberg, K., Farhat, C., 2008. A Compact Proper Orthogonal Decomposition Basis for Optimization-Oriented Reduced-Order Models. AIAA Paper 5964, 10-12.

[16] Carlberg, K., Farhat, C., 2011. A low-cost, goal-oriented compact proper orthogonal decompositionbasis for model reduction of static systems. International Journal for Numerical Methods in Engineering 86 (3), 381402 .

[17] Chaturantabut, S., Sorensen, D., 2009. Application of POD and DEIM on Dimension Reduction of Nonlinear Miscible Viscous Fingering in Porous Media.

[18] Chaturantabut, S., Sorensen, D., 2010. Discrete empirical interpolation for nonlinear model reduction. In: Decision and Control, 2009 held jointly with the 2009 28th Chinese Control Conference. CDC/CCC 2009. Proceedings of the 48th IEEE Conference on. IEEE, pp. 4316-4321. 
[19] Cook, R., 1995. Finite element modeling for stress analysis. John Wiley and Sons., New York.

[20] Couégnat, G., 2008. Approche multiéchelle du comportement mécanique de matériaux composites à renfort tissé. Ph.D. thesis, Université Sciences et Technologies-Bordeaux I.

[21] Cremonesi, M., Néron, D., Guidault, P.-A., Ladevèze, P., 2013. A pgdbased homogenization technique for the resolution of nonlinear multiscale problems. Computer Methods in Applied Mechanics and Engineering 267, 275-292.

[22] de Souza Neto, E., Feijóo, R., 2006. Variational foundations of multiscale constitutive models of solid: small and large strain kinematical formulation. LNCC Research \& Development Report 16.

[23] DeVore, R., Iserles, A., Suli, E., 2001. Foundations of computational mathematics. Cambridge Univ Pr.

[24] Drago, A., Pindera, M., 2007. Micro-macromechanical analysis of heterogeneous materials: Macroscopically homogeneous vs periodic microstructures. Composites science and technology 67 (6), 1243-1263.

[25] Dvorak, G., Wafa, A., Bahei-El-Din, Y., 1994. Implementation of the transformation field analysis for inelastic composite materials. Computational Mechanics 14 (3), 201-228.

[26] Efendiev, Y., Galvis, J., Gildin, E., 2012. Local-global multiscale model reduction for flows in high-contrast heterogeneous media. Journal of Computational Physics.

[27] Efendiev, Y., Galvis, J., Thomines, F., 2012. A systematic coarse-scale model reduction technique for parameter-dependent flows in highly heterogeneous media and its applications.

[28] Everson, R., Sirovich, L., 1995. Karhunen-Loeve procedure for gappy data. Journal of the Optical Society of America A 12 (8), 1657-1664.

[29] Feyel, F., Chaboche, J., 2000. Fe-2 multiscale approach for modelling the elastoviscoplastic behaviour of long fibre sic/ti composite materials. Computer methods in applied mechanics and engineering 183 (3), 309330 . 
[30] Fish, J., Shek, K., Pandheeradi, M., Shephard, M., 1997. Computational plasticity for composite structures based on mathematical homogenization: Theory and practice. Computer Methods in Applied Mechanics and Engineering 148 (1-2), 53-73.

[31] Galbally, D., Fidkowski, K., Willcox, K., Ghattas, O., 2010. Non-linear model reduction for uncertainty quantification in large-scale inverse problems. International Journal for Numerical Methods in Engineering 81 (12), 1581-1608.

[32] Geers, M., Kouznetsova, V., Brekelmans, W., 2010. Multi-scale computational homogenization: Trends and challenges. Journal of computational and applied mathematics 234 (7), 2175-2182.

[33] Grepl, M., Maday, Y., Nguyen, N., Patera, A., 2007. Efficient reducedbasis treatment of nonaffine and nonlinear partial differential equations. Mathematical Modelling and Numerical Analysis 41 (3), 575-605.

[34] Gross, D., Seelig, T., 2011. Fracture mechanics: with an introduction to micromechanics. Springer.

[35] Hernández, J. A., Oliver, J., Huespe, A., Caicedo, M., 2012. Highperformance model reduction procedures in multiscale simulations. Monograph CIMNE (ISBN:978-84-9939640-6-1).

URL http://centrovnet. cimne. upc.edu/cvdata/cntr7/dtos/img/mdia/Downloads/MONO

[36] Hoffman, J. D., 2001. Numerical Methods for Engineers and Scientists. Marcel Dekker.

[37] Hogben, L., 2006. Handbook of linear algebra. Chapman \& Hall/CRC.

[38] Hu, Y., Hwang, J., Perry, S., 2002. Handbook of neural network signal processing. The Journal of the Acoustical Society of America 111, 2525.

[39] Kim, T., James, D., 2009. Skipping steps in deformable simulation with online model reduction. In: ACM SIGGRAPH Asia 2009 papers. ACM, pp. 1-9.

[40] Kouznetsova, V., 2002. Computational homogenization for the multiscale analysis of multi-phase materials. 
[41] Krysl, P., Lall, S., Marsden, J., 2001. Dimensional model reduction in non-linear finite element dynamics of solids and structures. International Journal for Numerical Methods in Engineering 51 (4), 479-504.

[42] Kunisch, K., Volkwein, S., 2010. Optimal snapshot location for computing pod basis functions. ESAIM: Mathematical Modelling and Numerical Analysis 44 (3), 509.

[43] Li, Z., Wen, B., Zabaras, N., 2010. Computing mechanical response variability of polycrystalline microstructures through dimensionality reduction techniques. Computational Materials Science 49 (3), 568-581.

[44] Lovasz, L., Pelikan, J., Vesztergombi, K., 2003. Discrete Mathematics: Elementary and Beyond. Springer.

[45] Lubliner, J., 1990. Plasticity Theory. McMillan, New York.

[46] Maday, Y., Nguyen, N., Patera, A., Pau, G., 2007. A general, multipurpose interpolation procedure: the magic points.

[47] Maday, Y., Patera, A., Turinici, G., 2002. Reliable real-time solution of parametrized partial differential equations: Reduced-basis output bound methods.

[48] Michel, J., Moulinec, H., Suquet, P., 1999. Effective properties of composite materials with periodic microstructure: a computational approach. Computer methods in applied mechanics and engineering 172 (14), 109-143.

[49] Michel, J., Suquet, P., 2003. Nonuniform transformation field analysis. International journal of solids and structures 40 (25), 6937-6955.

[50] Michel, J., Suquet, P., 2004. Computational analysis of nonlinear composite structures using the nonuniform transformation field analysis. Computer methods in applied mechanics and engineering 193 (48-51), $5477-5502$.

[51] Monteiro, E., Yvonnet, J., He, Q., 2008. Computational homogenization for nonlinear conduction in heterogeneous materials using model reduction. Computational Materials Science 42 (4), 704-712. 
[52] Montgomery, D., Runger, G., 2010. Applied statistics and probability for engineers. Wiley.

[53] Nguyen, N., 2008. A multiscale reduced-basis method for parametrized elliptic partial differential equations with multiple scales. Journal of Computational Physics 227 (23), 9807-9822.

[54] Nguyen, N., Patera, A., Peraire, J., 2008. A best points interpolation method for efficient approximation of parametrized functions. Int. J. Numer. Meth. Engng 73, 521-543.

[55] Quarteroni, A., Sacco, R., Saleri, F., 2000. Numerical Mathematics. Springer, New York.

[56] Roussette, S., Michel, J., Suquet, P., 2009. Nonuniform transformation field analysis of elastic-viscoplastic composites. Composites Science and Technology 69 (1), 22-27.

[57] Rozza, G., 2009. Reduced basis methods for stokes equations in domains with non-affine parameter dependence. Computing and Visualization in Science 12 (1), 23-35.

[58] Ryckelynck, D., 2005. A priori hyperreduction method: an adaptive approach. Journal of computational physics 202 (1), 346-366.

[59] Ryckelynck, D., 2009. Hyper-reduction of mechanical models involving internal variables. International Journal for Numerical Methods in Engineering 77 (1), 75-89.

[60] Salomon, D., 2004. Data compression: the complete reference. SpringerVerlag New York Incorporated.

[61] Simo, J. C., Hughes, T. J. R., 1998. Computational inelasticity. Springer, New York.

[62] Yvonnet, J., He, Q., 2007. The reduced model multiscale method (R3M) for the non-linear homogenization of hyperelastic media at finite strains. Journal of Computational Physics 223 (1), 341-368. 